\title{
NUMERICAL INVESTIGATION ON THE EFFECT OF HOLE IMPERFECTION ON FILM COOLING PERFORMANCE
}

\author{
by \\ Taha Rezzag \\ Bachelor of Engineering, Ryerson University, 2017

\begin{abstract}
A thesis
presented to Ryerson University

in partial fulfillment of the

requirements for the degree of

Master of Applied Science

in the program of

Aerospace Engineering
\end{abstract}

Toronto, Ontario, Canada, 2019

(C) Taha Rezzag, 2019 


\section{AUTHOR'S DECLARATION FOR ELECTRONIC SUBMISSION OF A THESIS}

I hereby declare that I am the sole author of this thesis. This is a true copy of the thesis, including any required final revisions, as accepted by my examiners.

I authorize Ryerson University to lend this thesis to other institutions or individuals for the purpose of scholarly research.

I further authorize Ryerson University to reproduce this thesis by photocopying or by other means, in total or in part, at the request of other institutions or individuals for the purpose of scholarly research. I understand that my thesis may be made electronically available to the public. 


\title{
NUMERICAL INVESTIGATION ON THE EFFECT OF HOLE IMPERFECTION ON FILM COOLING PERFORMANCE
}

\author{
Taha Rezzag \\ Master of Applied Science, Aerospace Engineering, Ryerson \\ University, Toronto (2019)
}

\begin{abstract}
Film cooling holes in turbine blades are manufactured using different techniques, such as electro discharge, electro chemical and laser percussion drilling. The laser percussion drilling is the fastest one, making it a very attractive technique to use. However, some of the metal that has been melted by the laser solidifies inside the hole creating clumps that can reach up to $25 \%$ of the hole diameter. In order to comprehend the technique's influence on film cooling effectiveness, the hole imperfections produced by laser drilling has been modeled as a discrete inner half-torus located at a specific location inside the hole.

Film cooling thermal and hydrodynamic fields were predicted using various turbulence models combined with wall functions and the enhanced wall treatment. The k-omega SST model (for blowing ratios of 0.45 and 0.90) and realizable k-epsilon model combined with the enhanced wall treatment (for blowing ratio of 1.25) were chosen as results were in good agreement with the available experimental data from literature. The effect of imperfection position is studied at 4 different locations (1D, 2D, 3D and 4D) inside the hole measured from the hole leading edge, for three blowing ratios $(0.45,0.90$ and 1.25$)$ and a density ratio of 1. Effectiveness results for a blowing ratio of 0.45 reveal that the centerline effectiveness is improved as the imperfection is located farther from the hole exit. Compared to the perfect hole, the locations of $1 \mathrm{D}$ and $2 \mathrm{D}$ show a deterioration in the centerline effectiveness while the locations of $3 \mathrm{D}$ and $4 \mathrm{D}$ show an improvement from $\mathrm{x} / \mathrm{D}=0$ to 10 . Similar trends for the $1 \mathrm{D}$ and $2 \mathrm{D}$ locations can be seen for a blowing ratio of 0.90 where the centerline effectiveness is deteriorated. Furthermore, for a blowing ratio of 1.25, all imperfection locations show that a better film cooling performance is obtained for $\mathrm{x} / \mathrm{D}=0$ to 4 compared to the perfect hole but then deteriorates slightly onwards.

The present investigation also evaluates the influence of hole inclination angle with a hole imperfection on film cooling performance. Three hole inclination angles were investigated: $35^{\circ}, 45^{\circ}$ and $55^{\circ}$. Centerline effectiveness plots reveal a maximum effectiveness deterioration of $89 \%$ for a blowing ratio of 0.90 in the vicinity of the hole exit. Dimensionless temperature contours show that the jet produced in the presence of an imperfection is much more compact causing the counter rotating vortex pair to be closer to each other.
\end{abstract}


The final investigation of the present work evaluates the influence of imperfection shape and size on film cooling performance. A circular and rectangular profile imperfections were investigated at obstruction sizes of $26.3 \%, 35 \%$ and $40 \%$. Centerline effectiveness plots reveal a deterioration of $262.5 \%, 533.2 \%$ and $735.7 \%$ in effectiveness compared the perfect case at $26.3 \%, 35 \%$ and $40 \%$ obstructions respectively for a blowing ratio of 0.9 at a dimensionless distance of 10 downstream of the hole exit. Dimensionless temperature contour reveal that the lateral spreading of the coolant is more affected by imperfection shape at the location of $\mathrm{x} / \mathrm{D}=2$ where the circular shaped imperfection provides better laterally averaged effectiveness than the rectangular shaped imperfection especially of the $35 \%$ obstruction size. 


\section{ACKNOWLEDGMENTS}

I would like to extend my deepest thanks and gratitude to my thesis advisor, Dr. Bassam Jubran, for teaching me the fundamentals of engineering during my undergraduate studies and guiding me through my graduate endeavors at Ryerson University. Dr. Jubran introduced me to the field of computational fluid dynamics and thermofluids, and has believed in me and asks for me to do the same. He also trained me to think critically about scientific problems and be an independent researcher. What a great mentor to have! I am honoured for the great mentorship and friendship that Dr. Jubran has given me for the last eight years at Ryerson.

My journey at Ryerson has broadened my scope thanks to the aerospace engineering faculty and colleagues that put tremendous effort in effectively teaching and helping students. My thanks and gratitude go especially to my committee members, Dr. Goetz Bramasfeld, Dr. Hekmat Alighanbari and Dr. Jeffery Yokota.

Last but not least, my thanks go to my colleague and friend Sana Abdalsalam for spending countless hours helping me in hard times and looking out for me when a paper related to my thesis was published. Being her colleague and friend is indeed an honour. 


\title{
DEDICATION
}

\author{
$\mathcal{I}$ dedicate this thesis ta my parents \\ Latifa and Yaucef, \\ wha instilled in me the wirtues of patience, perseverance and cammitment and relentlessly \\ encauraged me ta strive far excellence.
}




\section{TABLE OF CONTENTS}

AUTHOR'S DECLARATION FOR ELECTRONIC SUBMISSION OF A THESIS ............ ii

ABSTRACT ............................................................................................................................................ iii

ACKNOWLEDGMENTS ........................................................................................................... v

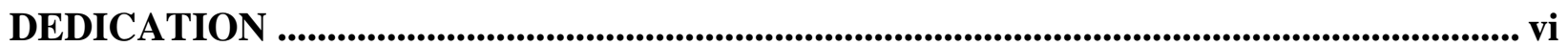

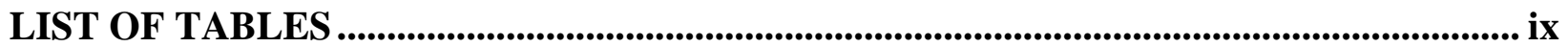

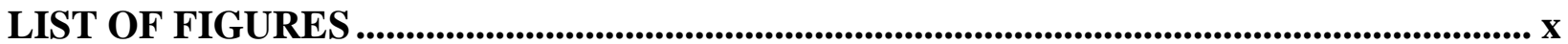

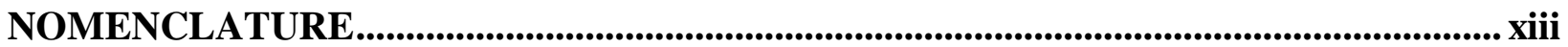

I. INTRODUCTION ............................................................................................................................... 1

Gas Turbines and Brayton Cycle ………………………................................................ 2

Technics to Increase Inlet Temperatures........................................................................... 4

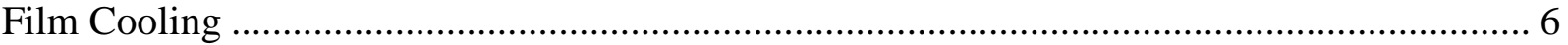

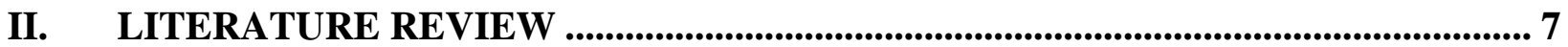

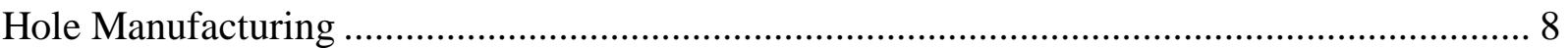

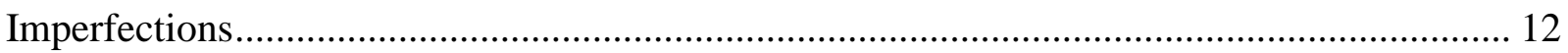

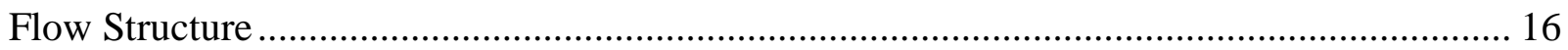

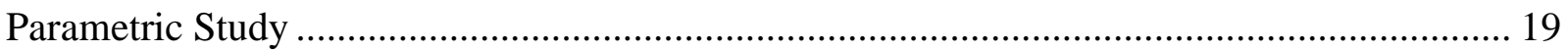

III. PROBLEM STATEMENT ….............................................................................................. 21

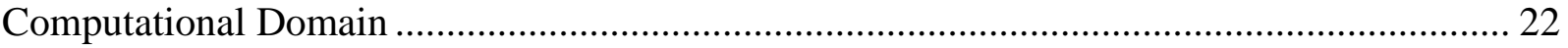

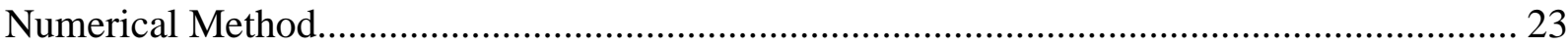

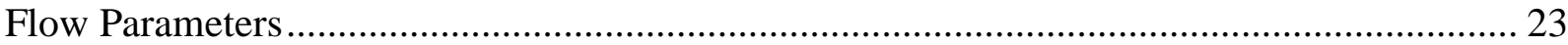

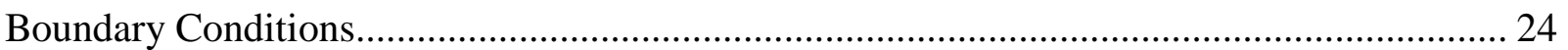

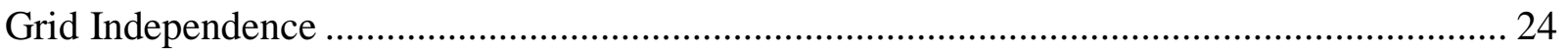

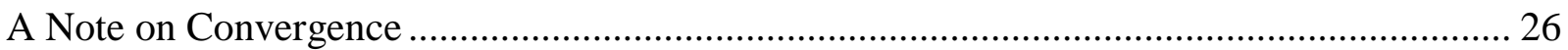


IV. RESULTS AND DISCUSSION ................................................................................... 27

Validation and Turbulence Modelling ……………………............................................... 28

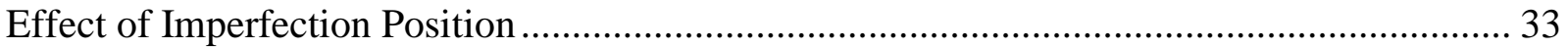

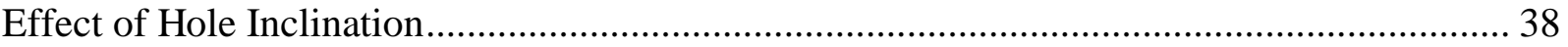

Effect of Imperfection Shape and Percent Obstruction........................................................... 45

V. CONCLUSIONS AND FUTURE RECOMMENDATIONS ....................................... 53

APPENDIX- TURBULENCE MODELING RESULTS .....................................................55

Perfect Case

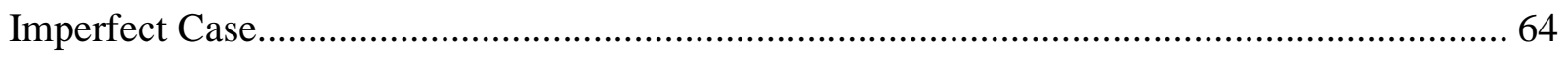

REFERENCES.......................................................................................................................... 73 


\section{LIST OF TABLES}

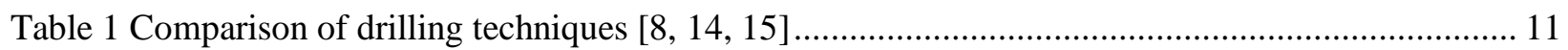

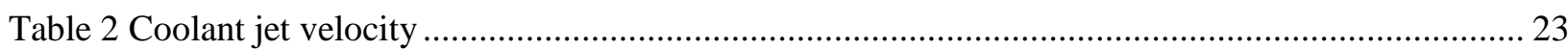

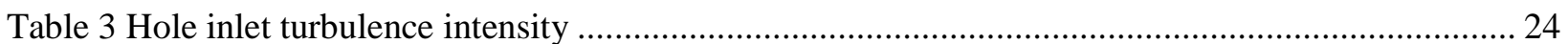




\section{LIST OF FIGURES}

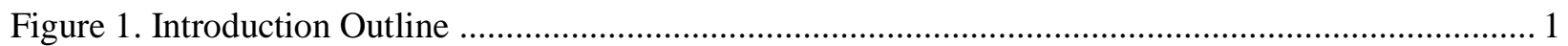

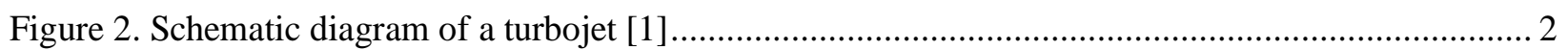

Figure 3. a) Air-Standard Gas Turbine Cycle b) Air-Standard Ideal Brayton Cycle [2]........................... 3

Figure 4. Variation of Turbine Entry Temperature Over Recent Years [6] ............................................ 5

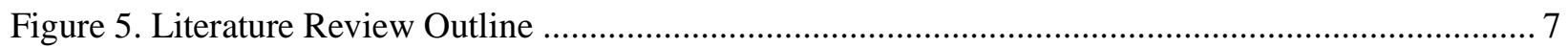

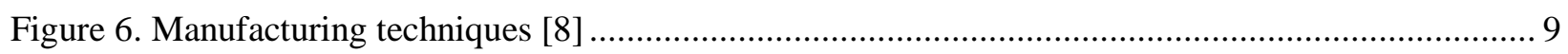

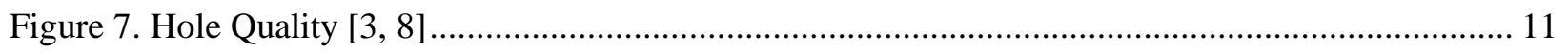

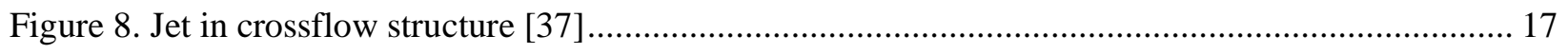

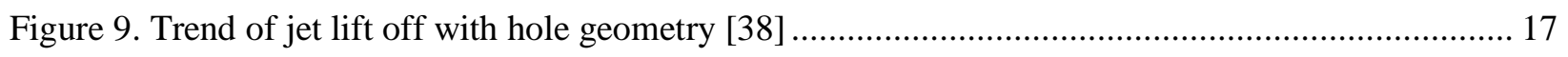

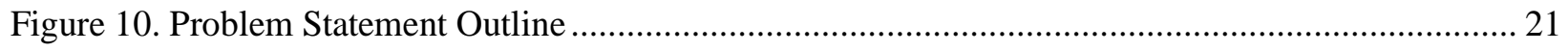

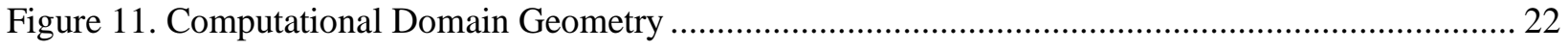

Figure 12. Geometry of the Perfect and Imperfect Hole …............................................................ 22

Figure 13. Computational Domain Boundary Conditions ….............................................................. 24

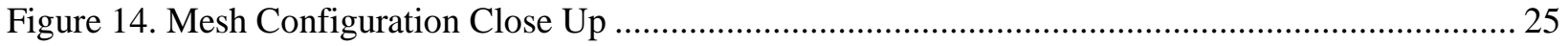

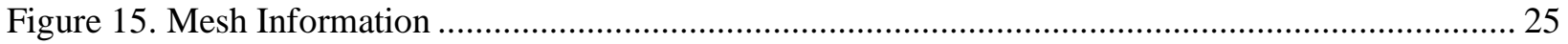

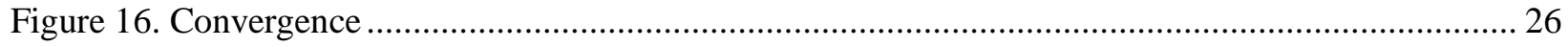

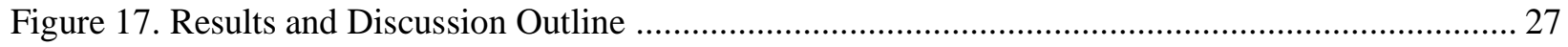

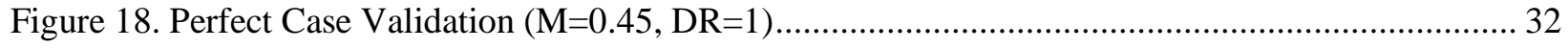

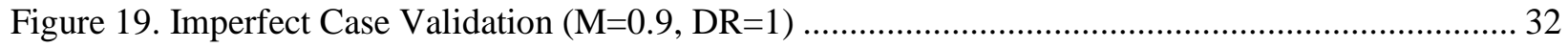

Figure 20. Laterally Averaged Effectiveness- Effect of Imperfection Position ....................................... 35

Figure 21. Centerline Effectiveness- Effect of Imperfection Position ................................................... 36

Figure 22. Dimensionless Temperature Contours and Velocity Vectors at $\mathrm{x} / \mathrm{D}=1$ for $\mathrm{M}=0.45$ for Perfect

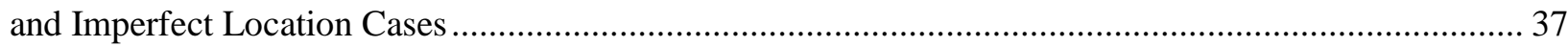

Figure 23. Laterally Averaged Film Cooling Effectiveness; Perfect (Black) and Imperfect (Blue) Cases 41

Figure 24. Centerline Film Cooling Effectiveness; Perfect (Black) and Imperfect (Blue) Cases ............. 42

Figure 25. Dimensionless Temperature Contours and Velocity Vectors at $\mathrm{x} / \mathrm{D}=1$ for $\mathrm{M}=0.9$ for Perfect (left) and Imperfect (right) Cases....

Figure 26. Dimensionless Temperature Contours and Velocity Vectors at $\mathrm{x} / \mathrm{D}=5$ for $\mathrm{M}=0.9$ for Perfect (left) and Imperfect (right) Cases.....

Figure 27. Laterally Averaged Film Cooling Effectiveness; Perfect (Black), Imperfect Circular (Blue) and Rectangular (Red) Cases. 48 
Figure 28. Centerline Film Cooling Effectiveness; Perfect (Black) and Imperfect Circular (Blue) and Rectangular (Red) Cases.

Figure 29. Centerline and Laterally Averaged Film Cooling Effectiveness for Circular Imperfection;

$26.3 \%$ (Black), $35 \%$ (Blue) and 40\% (Red) 50

Figure 30. Centerline and Laterally Averaged Film Cooling Effectiveness for Rectangular Imperfection;

$26.3 \%$ (Black), 35\% (Blue) and 40\% (Red)

Figure 31. Dimensionless Temperature Contours and Velocity Vectors at $\mathrm{x} / \mathrm{D}=2$ for $\mathrm{M}=0.45$ for Circular (left) and Rectangular (right) Shaped Imperfections at Various Sizes 52

Figure 32. Laterally Averaged Film Cooling Effectiveness at a Blowing Ratio of 0.45 and Density Ratio of 1 at $\mathrm{Y}+$ of 30 for the Perfect Case. .55

Figure 33. Centerline Film Cooling Effectiveness at a Blowing Ratio of 0.45 and Density Ratio of 1 at Y+ of 30 for the Perfect Case. 56

Figure 34. Film Cooling Effectiveness at a Blowing Ratio of 0.45 and Density Ratio of 1 at $Y+$ of 1 for the Perfect Case

Figure 35. Laterally Averaged Film Cooling Effectiveness at a Blowing Ratio of 0.90 and Density Ratio of 1 at $\mathrm{Y}+$ of 30 for the Perfect Case... 58

Figure 36. Centerline Film Cooling Effectiveness at a Blowing Ratio of 0.90 and Density Ratio of 1 at $\mathrm{Y}+$ of 30 for the Perfect Case.

Figure 37. Film Cooling Effectiveness at a Blowing Ratio of 0.90 and Density Ratio of 1 at $\mathrm{Y}+$ of 1 for the Perfect Case

Figure 38. Laterally Averaged Film Cooling Effectiveness at a Blowing Ratio of 1.25 and Density Ratio of 1 at $\mathrm{Y}+$ of 30 for the Perfect Case.

Figure 39. Centerline Film Cooling Effectiveness at a Blowing Ratio of 1.25 and Density Ratio of 1 at $\mathrm{Y}+$ of 30 for the Perfect Case.

Figure 40. Film Cooling Effectiveness at a Blowing Ratio of 1.25 and Density Ratio of 1 at $\mathrm{Y}+$ of 1 for the Perfect Case

Figure 41. Laterally Averaged Film Cooling Effectiveness at a Blowing Ratio of 0.45 and Density Ratio of 1 at $\mathrm{Y}+$ of 30 for the Imperfect Case

Figure 42. Centerline Film Cooling Effectiveness at a Blowing Ratio of 0.45 and Density Ratio of 1 at $\mathrm{Y}+$ of 30 for the Imperfect Case

Figure 43. Film Cooling Effectiveness at a Blowing Ratio of 0.45 and Density Ratio of 1 at $Y+$ of 1 for the Imperfect Case

Figure 44. Laterally Averaged Film Cooling Effectiveness at a Blowing Ratio of 0.90 and Density Ratio of 1 at $\mathrm{Y}+$ of 30 for the Imperfect Case 
Figure 45. Centerline Film Cooling Effectiveness at a Blowing Ratio of 0.90 and Density Ratio of 1 at Y+ of 30 for the Imperfect Case

Figure 46. Film Cooling Effectiveness at a Blowing Ratio of 0.90 and Density Ratio of 1 at Y+ of 1 for the Imperfect Case

Figure 47. Laterally Averaged Film Cooling Effectiveness at a Blowing Ratio of 1.25 and Density Ratio of 1 at $\mathrm{Y}+$ of 30 for the Imperfect Case

Figure 48. Centerline Film Cooling Effectiveness at a Blowing Ratio of 1.25 and Density Ratio of 1 at Y+ of 30 for the Imperfect Case

Figure 49. Film Cooling Effectiveness at a Blowing Ratio of 1.25 and Density Ratio of 1 at $\mathrm{Y}+$ of 1 for the Imperfect Case 72 


\section{NOMENCLATURE}
Alphabetical
D
Diameter
DR
Density ratio
L
Length
l
Turbulence length scale
$k$
Specific heat ratio; Kinetic energy of turbulent
fluctuations per unit mass
M
Blowing ratio
$\dot{Q}_{\text {in }}$
Heat added
$\dot{Q}_{\text {out }}$
Heat rejected
$p$
$\mathrm{P} / \mathrm{D}$
Pressure
$\operatorname{Re}$
Pitch to diameter ratio
$T$
Reynolds number
Temperature
$V, \mathrm{U}$
Velocity
$y^{+}$
Normalized distance from wall
$\mathrm{x}, \mathrm{y}, \mathrm{z}$
Cartesian coordinate

\section{Greek}

$\alpha$

$$
\beta, \beta^{*}, \sigma, \sigma^{*}
$$

$\eta$

$\bar{\eta}$

$\theta$

$\Theta$

$\rho$

$\varepsilon$

$\mu$

$v$

$\omega$
Thermal diffusivity; Closure coefficient

Closure coefficients

Film cooling effectiveness/ Thermal efficiency (with subscript $\mathrm{T}$ )

Laterally averaged film cooling effectiveness

Dimensionless temperature

Mean temperature

Density

Turbulent dissipation rate

Dynamic viscosity

Kinematic viscosity

Specific dissipation rate 


\section{Subscripts}

1

2

3

4

aw

c

$\mathrm{T}$

$\infty$

\section{Acronyms}

AGBT

CRVP

ECM

EDM

ECDM

RANS

RSM

SIMPLEC

SST

TBC
Compressor inlet

Compressor outlet

Turbine inlet

Turbine outlet

Adiabatic wall

Coolant

Thermal

Freestream

Modellschaufel der Arbeitsgemeinschaft

Hochttemperaturgasturbine (AG Turbo)

Schaufeltyp B (Shovel Model of the Working

Group Temperature Gas Turbine (Turbo AG)

Bucket type B)

Counter-rotating vortex pair

Electrochemical discharge machining

Electrodischarge machining

Electrochemical discharge machining

Reynolds-averaged Navier Stokes

Reynolds-stress model

Semi-Implicit Method for Pressure-Linked

Equations-Consistent

Shear stress transport

Thermal barrier coating 


\section{INTRODUCTION}

The purpose of this chapter is to refresh the reader's knowledge on some relevant topics before embarking into the main focus of this thesis. A thorough discussion of gas turbines and their constituents along with a full Brayton cycle analysis of jet engines is first considered. This section aims to present to the reader a design concern that arises with gas turbine engines possible engineering advancements and achievements made into addressing this concern will then be discussed. Finally, among the various technics used, film cooling, will be introduced along with the key parameters used to measure its performance. Figure 1 summarizes the outline of this chapter.

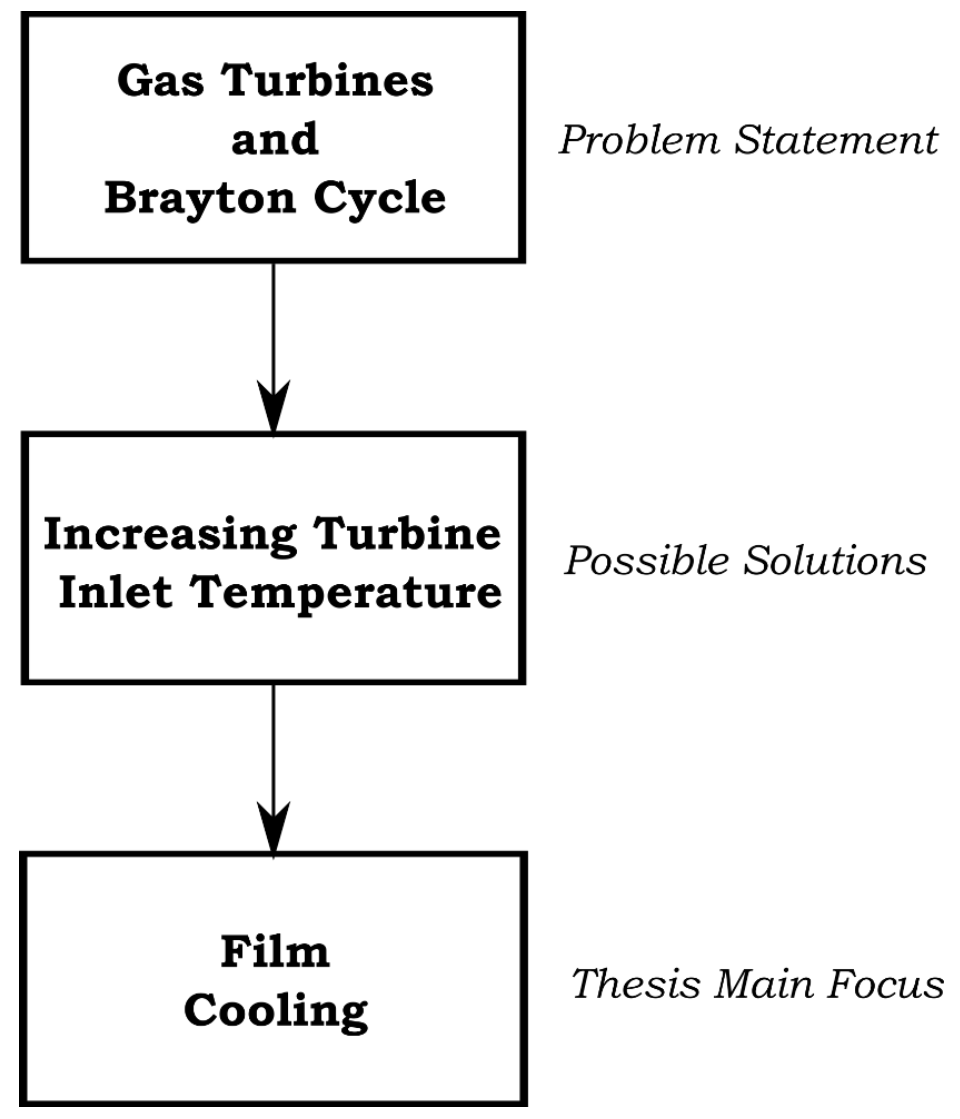

Figure 1. Introduction Outline 


\section{Gas Turbines and Brayton Cycle}

Gas turbine engines are propulsion systems that increase the kinetic energy of air passing through them. In order to produce an important velocity change, the engine is designed to consist of mainly three components where the working fluid is subjected to various processes. Initially, incoming air from the atmosphere passes through the first component of the engine, the compressor, where it's pressure is considerably increased through multiple stages. This is done so that the following processes, power extraction, will be performed more efficiently. Gas turbine compressors are of two types, centrifugal and axial, where each have advantages over the other. Centrifugal compressors increase air pressure caused by a rotational motion and are made up of three components: an impeller, a diffuser and a compressor manifold. Axial compressors on the other hand increases air pressure through a series of rotating rotor blades and stationary stator vanes. Most compressors used in gas turbines comprise of multiple low and high pressure stages. After the air reaches a prescribed pressure, it then goes into the second component of the engine, the combustion chamber, where less than one half of the pressurized air entering the burner is mixed with fuel and then ignited. The remaining air absorbs some of the heat generated and cools the walls of the combustion chamber. In order for combustion to occur, set by pressure, an average of total air to fuel ratio where about 15 parts are used in the combustion process is used for newer types of engines. As the fuel-air mixture gets ignited, it will expend through the third component, the turbine, where it will pass through multiple stages extracting kinetic energy. The energy is then converted to shaft horsepower where three-fourths of it is used to drive the compressor; it is important to note that the turbine is connected to the compressor through a shaft. The conversion of kinetic energy to mechanical shaft energy occurs through the interaction of the turbine stator and wheel rotor. The hot gases are then exhausted through a nozzle where the velocity is considerably faster than the air coming into the compressor creating a force acting forward according to Newton's second law of motion. A schematic diagram of a turbojet, see Figure 2, highlights the main components discussed above.

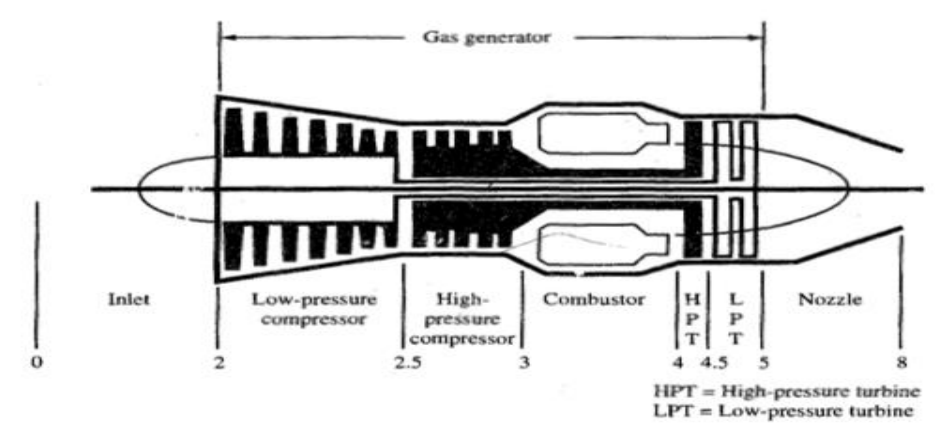

Figure 2. Schematic diagram of a turbojet [1] 
In order to investigate and monitor the performance of gas turbine engines, thermodynamics concepts such as the Brayton cycle have been developed. The Brayton cycle gives insight into what parameters affects the efficiency of the engine. More specifically, the air-standard ideal Brayton cycle will be more relevant to the current discussion. The ideal "air-standard analysis" an idealization must be made in order to analyze this system. The idealization consists of three assumptions [2]:

1. Air is the fluid of interest and behaves as an ideal gas.

2. The temperature rise experienced during the combustion chamber stage is assumed to be caused by a heat transfer from an external source.

3. Irreversibilities are ignored.

Applying these assumptions, the exhaust at the turbine is returned to its original atmospheric properties closing the loop known as the air-standard ideal gas turbine cycle shown in Figure 3a.

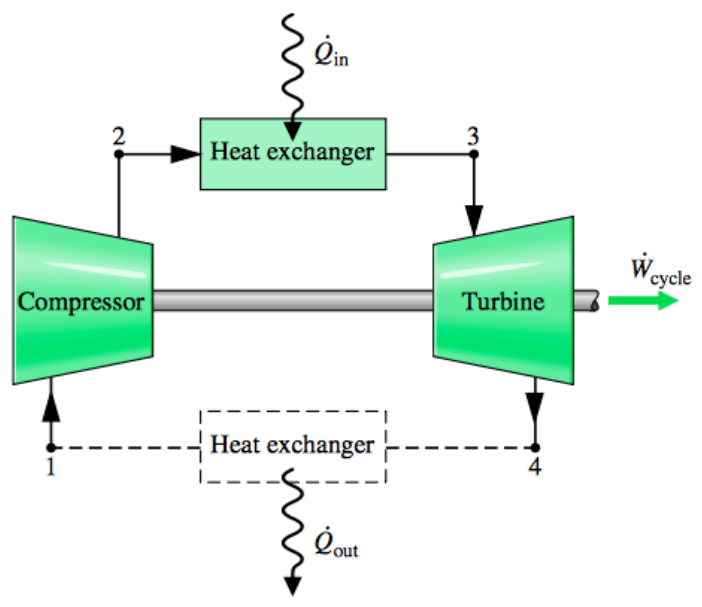

a)

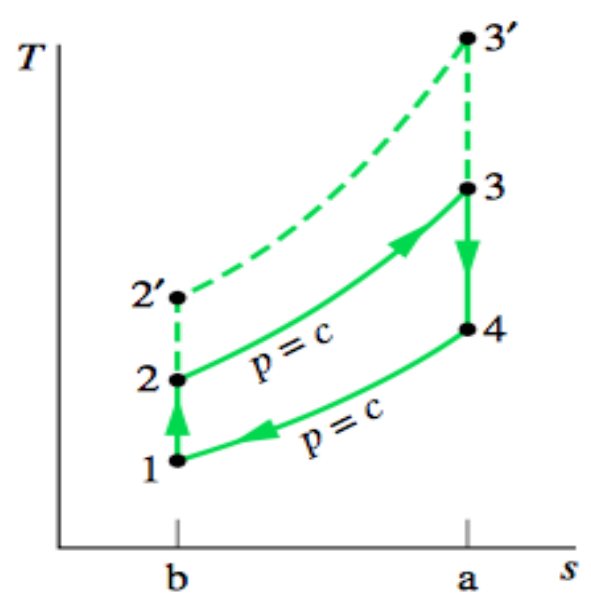

b)

Figure 3. a) Air-Standard Gas Turbine Cycle b) Air-Standard Ideal Brayton Cycle [2]

The air-standard ideal Brayton cycle is shown in the T-s graph, temperature vs. entropy, provided in figure $3 \mathrm{~b}$ where the area enclosed by b-2-3-4-b represents the heat added into the system per unit of mass, $\dot{Q}_{i n}$ in Figure $3 \mathrm{a}$, while the area enclosed by $\mathrm{b}-1-4-\mathrm{a}-\mathrm{b}$ represents the heat rejected out of the system per unit of mass, $\dot{Q}_{\text {out }}$ in Figure 3a. Therefore, the area enclosed by the contour is the net heat added into the system per unit of mass. The T-s diagram shows that as the compressor pressure ratio is increased, the cycle changes from 1-2-3-4-1 to 1-2'-3'-4-1 representing an increase in the heat added per unit mass. This increase in heat added represents an enhancement in the thermal efficiency of the gas turbine which can be quantified by the following equation 


$$
\eta_{T}=1-\frac{1}{\left(p_{2} / p_{1}\right)^{\frac{k-1}{k}}}
$$

Or,

$$
\eta_{T}=1-\frac{T_{1}}{T_{2}}
$$

Where $\eta_{T}$ is the thermal efficiency, $p_{1}$ is the compressor inlet pressure, $p_{2}$ is the compressor outlet pressure, $T_{1}$ is the compressor inlet temperature, $T_{2}$ is the compressor outlet temperature and $\mathrm{k}$ is the specific heat ratio. It can be observed from equation 1 that as the compressor pressure ratio is increased, the thermal efficiency of the gas turbine approaches closer to unity. As was stated above, an increase in compressor pressure ratio translates to a higher turbine inlet temperature. This introduces a main design constraint on how effective the gas turbine can be caused by metallurgical limits imposed by the turbine blades material.

\section{$\underline{\text { Technics to Increase Inlet Temperatures }}$}

In order to enable the turbine inlet temperature to reach high levels without risking a structural failure of the engine, two approaches are available. The first consists of developing new materials capable of withstanding high temperatures and stresses. This approach however is not practical as it is expensive and takes a long period of time to reach acceptable results. Materials known for their high temperature resistance, low heat conductivity, such as ceramics cannot withstand the stresses experienced in the turbine due their brittleness. The second approach consists of protecting current turbine blades by either coating them, cooling them or a combination of the two. This approach has proved to more practical for the time being until a better material that can fulfill the desired requirements is developed. Coating technologies applied to turbine blades such as thermal barrier coating (TBC) consist of applying two layers on the blades; the bond coat and the top coat. The bond coat is made of an oxidation-resistant alloy that serves as a rough surface for proper adhesion of the top coat. The layer above the bond coat, the top coat, is the one used for thermal barrier purposes and is made of ceramic [3]. A top coat of $250 \mu \mathrm{m}$ typically causes a temperature drop of $200 \mathrm{~K}$ across it [4]. Cooling technologies involving relatively cooler air retrieved from the compressor staged have also been used extensively in protecting turbine blades from hot gases. One of the earliest techniques, convection cooling, consisted of cooling the inner walls of turbine blades through cavities. Another inner cooling technique, jet impingement cooling, consists of internally targeting critical spots in turbine blades with jets of cooler air. The most vulnerable locations on turbine blades due to their exposure to extreme temperatures are the stagnation points; the leading and trailing edges [5]. Therefore, 
targeting critical locations increases cooling effectiveness while also decreasing the amount of coolant retrieved from the compressor. External cooling techniques, film cooling, will be the focus of this thesis and will be discussed in more detail in the next section. Before proceeding with a discussion on film cooling however, it is of interest to see the contributions of the technics discussed above on increasing the turbine inlet temperature over a period of 60 years (see Figure 4). It can be seen from the graph below that advancements in material temperature allowance from early 1950 to late 80 's has made minimal improvements. This emphasizes the point that protective and cooling techniques are essential in order to achieve considerable progress while at the same time developing new materials as was done in the early 90's. The graph shows that film cooling has allowed a turbine entry temperature rise of $67 \%$ and reach approximately $2000 \mathrm{~K}$ from an uncooled turbine inlet temperature of $1200 \mathrm{~K}$. Fortunately, the trend is still persisting as new materials and new cooling concepts are developed causing the gas turbine engine's efficiency to further improve.

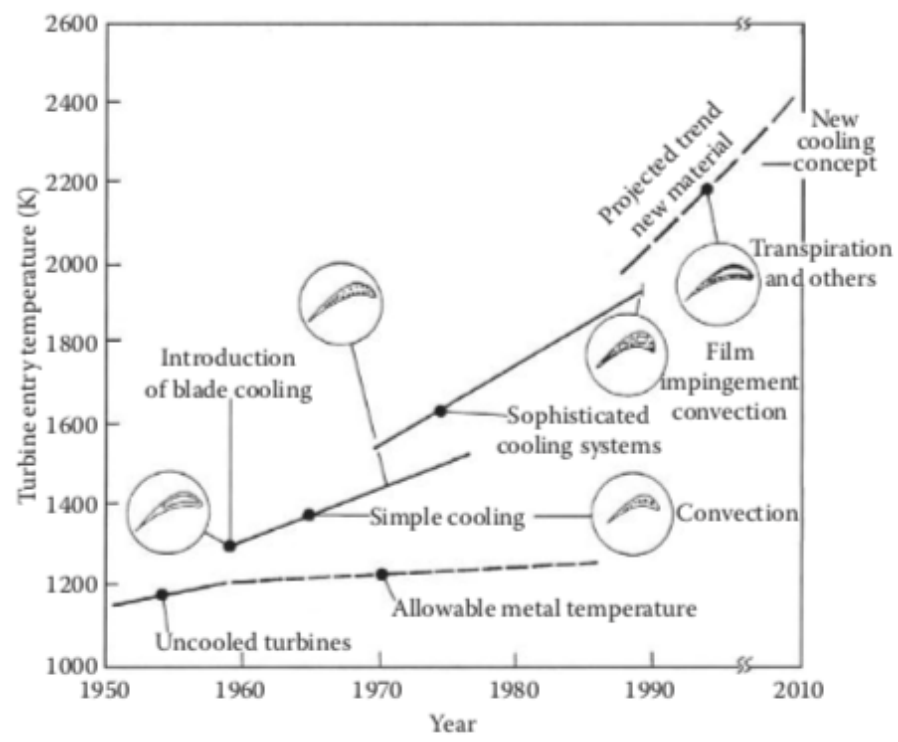

Figure 4. Variation of Turbine Entry Temperature Over Recent Years [6] 


\section{Film Cooling}

As the name suggests, the external cooling method involves a film of cooler air, relative to the mainstream, that is introduced by a coolant jet upstream through holes. Conceptually, the film lays and spreads on top of the surface of the blade forming a protective barrier between it and the incoming hot air allowing the heat to transfer mainly to the film. Jets in crossflow give rise to very complex flow physics that require careful study as high mixing occurs defeating the main purpose of the jet. This motivated further research in film cooling where individual parameters are studied to successfully manipulate the coolant jet in a desired fashion. Film cooling effectiveness is a parameter used to determine how well the film is protecting the blade and is a good measure of the performance of the film. The film cooling effectiveness relates the three main temperatures involved namely the temperature of the hot gases, the temperature of the coolant jet and the temperature of the turbine blade and is written as follows

$$
\eta=\frac{T_{\infty}-T_{a, w}}{T_{\infty}-T_{c}}
$$

Where $\eta$ is the film effectiveness, $\mathrm{T}_{\infty}$ is the mainstream temperature, $\mathrm{T}_{\mathrm{c}}$ is the coolant temperature and $T_{a, w}$ is the adiabatic wall temperature. When a location above the surface is concerned, $T$ instead of $T_{a, w}$, the film cooling effectiveness, $\eta$, becomes known as the dimensionless temperature $\theta$. Another important parameter, the blowing ratio, is defined as the product of coolant jet to mainstream density and velocity ratio as

$$
M=\frac{\rho_{c} V_{c}}{\rho_{\infty} V_{\infty}}
$$

Where $M$ is blowing ratio, $\rho_{\infty}$ is the mainstream density, $V_{\infty}$ is the mainstream velocity, $\rho_{c}$ is the exit coolant density and $V_{c}$ is the coolant exit velocity. More parameters used to study the effect of hole imperfections on film cooling performance will be investigated and explained in the following chapters. 


\section{LITERATURE REVIEW}

The effect of hole imperfections produced during the manufacturing process, laser percussion drilling, on the film cooling performance is the main focus of this thesis. Before boarding this topic, a discussion of the available literature on the various manufacturing techniques for drilling film cooling holes presently available is essential. Furthermore, the effects of imperfections and their locations, caused either by debris deposition or manufacturing processes, on film cooling effectiveness by will be discussed next. In order to gain a deeper insight into the influence of hole imperfections, a treatment of jet in cross flow structure is necessary. Finally, a discussion on the effects of various film cooling parameters that will be investigated in this thesis will conclude this chapter. Figure 5 summarizes the outline of this chapter.

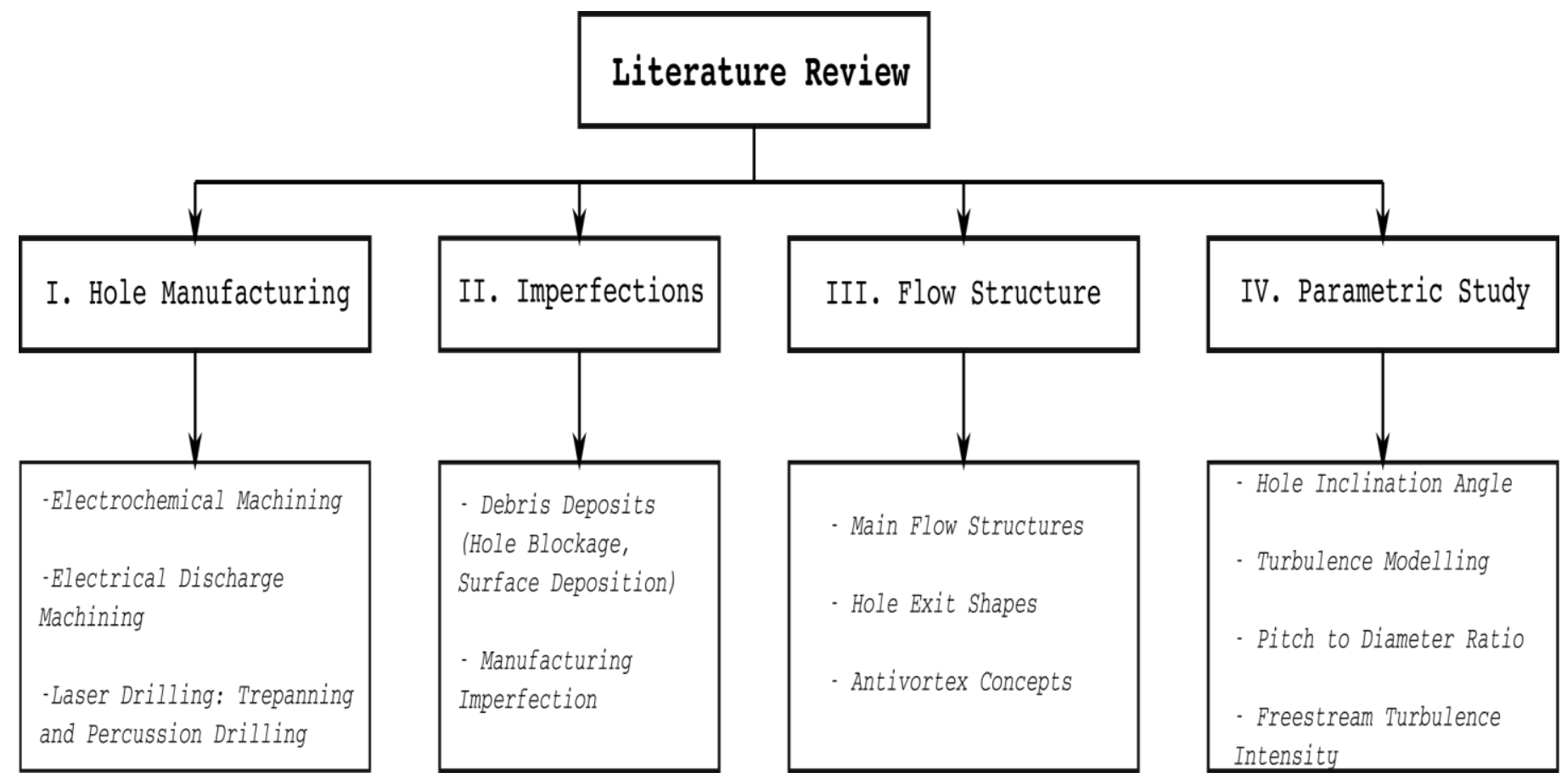

Figure 5. Literature Review Outline 


\section{$\underline{\text { Hole Manufacturing }}$}

Turbine blade and vanes can have anywhere between 25-200 film cooling discrete holes while the afterburner has 40,000 [7]. Using conventional manufacturing techniques on superalloys, such as punching and twisting, have proved to be both challenging and time consuming [8]. Therefore, it is important that these holes be manufactured in a fast pace while maintaining a certain degree of accuracy in order to reduce cost. Film cooling holes in turbine blades are manufactured using different techniques, such as electro discharge, electro chemical and laser percussion drilling. The laser percussion drilling method is the fastest one, making it a very attractive technique to use. However, some of the metal that has been melted by the laser solidifies inside the hole creating clumps that can reach $25 \%$ of the hole diameter [9]. The electrochemical machining (ECM) concept consists of removing material from a target surface by anodic dissolution [8]. This is done by connecting the surface to a power source such that it acts as an anode while the cutting tool acts as the cathode. A flow of electrolyte, generally dilute hydrochloric acid $\mathrm{HCl}$ [10], is then pumped through the cathode to remove the ions, see Figure 6a. Similarly, to the ECM, the electrodischarge machining (EDM) concept consists of connecting both the workpiece and tool to a power supply so that they become the anode and cathode respectively, see Figure 6b. However, instead of relying on an electrolyte supply to cause dissolution of the material, the station is immersed in a dielectric fluid, generally kerosene, where small electrical sparks between the cathode and anode are generated to erode the metal from the target surface producing a cavity [8]. Kulkarni [10] performed an experimental study on the electrochemical discharge machining (ECDM) process for microscopic holes. ECDM is a hybrid process comprising of both ECM and EDM. The heat generated to form the hole was found to dissipate rapidly through the workpiece away from the hole. However, maintaining such heat might adversely affect areas near the discharge location requiring the process to be completed through pulsations or a higher electrolyte flow. Ahmed [11] considered the various applications of both the EDM and ECM methods with a discussion on the advantages and disadvantages of the ECM method. EDM is commonly used for applications such as injector nozzle drilling and texturing of large surface areas. On the other hand, ECM is used to machine complex geometries on hard surfaces. The advantages of ECM consist of the following [11]:

- The cutting rate is not influenced by the hardness of the metal

- High length to diameter ratios are achieved

- Drilling multiple holes at the same time

- $\quad$ Cuts are smooth with no burrs

- Holes are metallurgically stress free

- Angles and intersecting holes are possible 
The disadvantages are as follows [11]:

- This method can only be used on stainless metals

- $\quad$ Slow method for drilling single holes

- Handling of corrosive chemical (i.e. electrolyte) which has adverse environmental effects [8]

- $\quad$ Some ECM methods use electrodes made of glass and can be broken easily

- Other ECM methods use very high voltage creating an electrical hazard and a corrosion problem

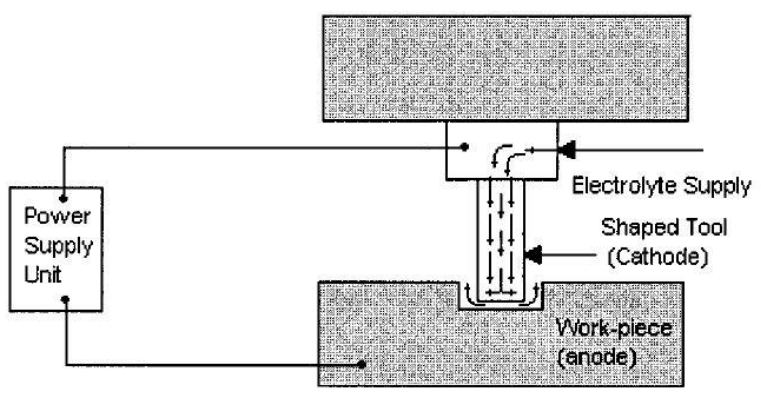

a) Electrochemical machining

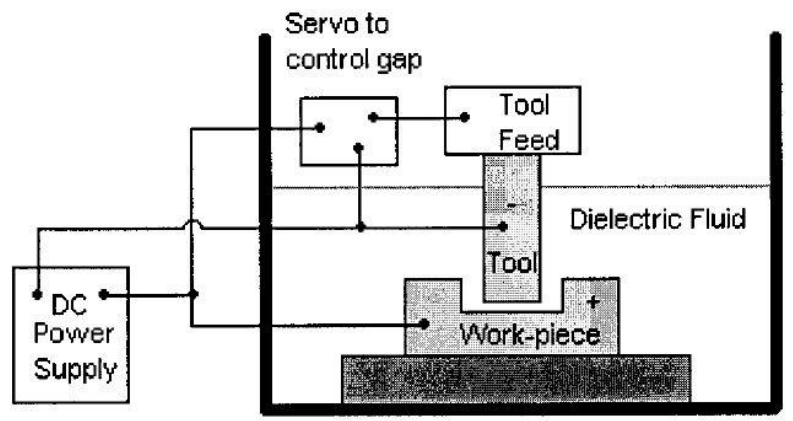

b) Electrical discharge machining

Figure 6. Manufacturing techniques [8]

Imperfections produced by laser drilling are the main focus of this thesis. This process consists of two methods namely; percussion and trepanning drilling. Trepanning drilling uses the laser, Nd-YAG is commonly used in the aerospace industry, to pierce the hole through the center and goes through the circumference in order to cut the desired profile. This method requires either the workpiece or the laser configuration to be able to rotate. Percussion drilling on the other hand consists of focusing and pulsating a laser beam to the desired target. The material is then removed through vaporizing and liquefying it. Moreover, this method does not require either the laser nor the workpiece the rotate. Laser drilling does have some disadvantages regarding hole quality and geometry despite having the ability to "drill on-thefly", or the ability to drill holes on moving surfaces. The hole quality issues associated with laser drilling include hole tapering, barreling, re-cast layers, spatter and micro-cracking. The term hole tapering refers the differing hole inlet and exit diameters while barreling refers to the shape of the hole walls caused by the laser. Re-cast layers' form inside the walls of the drilled holed due to solidified molten or vaporized material, see Figure 7a. When these molten particles are ejected from the hole and land on the surface nearby the hole exit, they form what is known as spatter, see Figure 7b. Spatter hurts the repeatability of closely spaced hole arrays as it may cause non-uniform absorption of the laser. Moreover, micro-cracking is witnessed inside the holes due to the thermal nature of the drilling process [8].

Naeem [7] conducted an experimental investigation on the performance of fiber delivered systems compared to direct beam delivery. It was found that a fiber delivered drilled had a few advantages over the 
direct beam delivery such as its lower cost, compactness, variable hole sizes, beam quality, hole quality, acute angles and drill on-the-fly with varying pulse frequencies.

Voisey and Clyne [3] performed experiments studying the effects of drilling film cooling holes through plasma sprayed thermal barrier coatings. Among the results retrieved from this investigation, it was found that laser drilling's main damage on TBCs is cracking at the bond coat and substrate interface. Additionally, it was concluded that laser drilling the workpiece from the metal side resulted in deeper holes compared to drilling the workpiece from the TBC side.

French et al. [12] experimentally investigated the dominant factors affecting the laser drilling process. Results of the investigation reveal that laser percussion drilling consists of three stages. The first stage involves the laser breaking the reflectivity of the surface and forming a plasma plume above it taking approximately ten microseconds to complete. The majority of the material will then be ejected in the forms of plasma and liquid conical sheets taking 50 milliseconds. The second stage involves material removal in ribbon or column like fashion ejecting in random directions. The final stage begins when the ejections transform to droplets of melted material. The last two stages take approximately 31-70 milliseconds to complete. It was found that the presence of assist gas and pressure are very important in determining hole quality.

French et al. [13] experimentally investigated the effect of laser drilling using a 400 micrometer optical fibre delivery system. It was found that a laser delivered through a 400 micrometer optical fiber can product good quality holes. Moreover, it has been shown that pulse shaping and focal positioning play an important role in determining hole quality. The shape of the pulse used was found to influence material removal efficiency. For instance, the ramping up pulse is more efficient in removing material than the square shaped pulse. It was found that for optical fibre delivered systems, focal positions above the surface produce higher quality holes compared to focal points located below the surface.

New hole manufacturing techniques such as the abrasive waterjet technology are being developed to address some of the disadvantages found in current techniques. Waterjet cutting is a very accurate method capable of drilling shaped holes as suggested by the film cooling literature. Moreover, waterjets can penetrate tough materials such as alloys and ceramics. However, this technique is still in the developmental stage as it requires an expensive facility and is detrimental for corrosive materials. Table 1 below compares the principle performance parameters of the main three manufacturing techniques discussed above. 


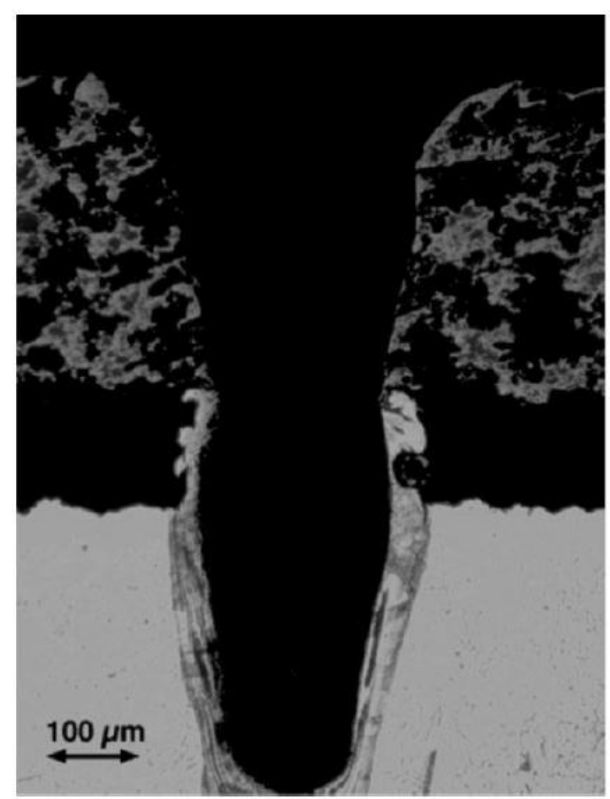

a) Recast

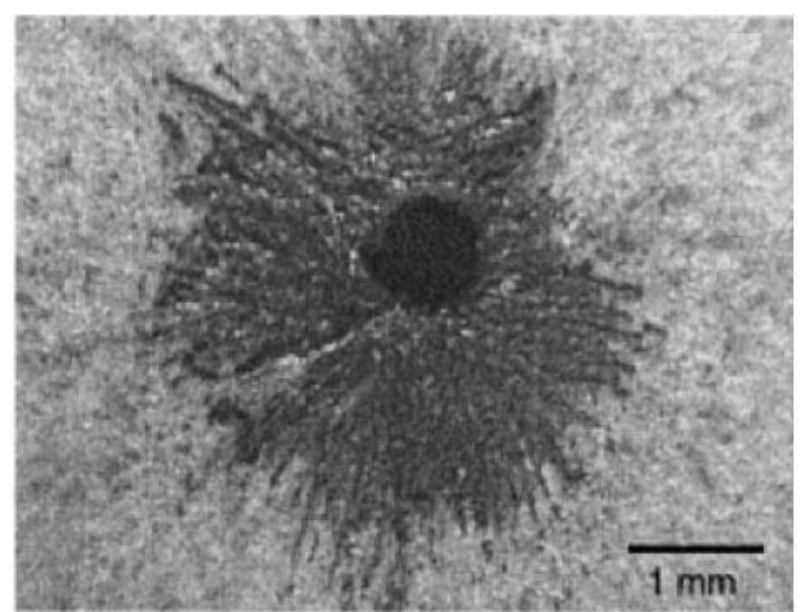

Substrate

Figure 7. Hole Quality [3, 8]

Table 1 Comparison of drilling techniques $[8,14,15]$

\begin{tabular}{|c|c|c|c|}
\hline & ECM & EDM & Laser \\
\hline Material & $\begin{array}{l}\text { Conductive materials } \\
\text { only; hard or soft }\end{array}$ & $\begin{array}{l}\text { Conductive materials } \\
\text { only; hard or soft }\end{array}$ & Any \\
\hline Tool wear & None & Significant & None \\
\hline Material removal rate & Low & Low* & High \\
\hline $\begin{array}{l}\text { Acute angles relative to } \\
\text { surface }\end{array}$ & No & Approx. $25^{\circ}$ & Approx. $15^{\circ}$ \\
\hline Deep holes & No & Yes & Yes \\
\hline $\begin{array}{l}\text { Minimum hole size, } \\
\text { mm }\end{array}$ & 0.75 & 0.3 & 0.1 \\
\hline Hole quality & $\begin{array}{l}\text { Accurate and good } \\
\text { repeatability }\end{array}$ & $\begin{array}{l}\text { Accurate and good } \\
\text { repeatability; re-cast } \\
\text { layer present }\end{array}$ & $\begin{array}{l}\text { Repeatability poor; } \\
\text { recast layer present }\end{array}$ \\
\hline
\end{tabular}




\section{Imperfections}

Imperfections forming in the air passages inside a gas turbine engine are caused either through debris depositions or manufacturing processes. Debris depositions are common in airports located in sandy areas or when an airplane cruises over an area laden with volcanic ash. The sand or ash will then be ingested inside the engine and accumulate inside and around the film cooling holes disturbing the jets' flow structure. Pan et al. [16] conducted a numerical investigation on the effect of hole partial blockage on film cooling effectiveness. The partial blockage was modeled as a tetrahedron apex and was placed at specific locations inside the hole. The locations chosen were the inlet, middle and exit of the hole where the blockage was placed at the leading, trailing or lateral position. It was found that the location of the partial blockage and its position has a significant effect on film cooling performance especially at the hole exit. A deterioration in film cooling effectiveness was witnessed for blockages located at the trailing edge or laterally of the hole. However, when the partial blockage was placed at the hole exit at the leading edge position the film cooling effectiveness was improved. It was also found that the size of the blockage amplified the deterioration, for the trailing edge and lateral positions, or the improvement, for the leading edge position, of the film cooling performance.

Zhou et al. [17] conducted a numerical investigation on the effect of blockage position and size, as used in [16], located in a blade's pressure side on film cooling effectiveness. The holes are integrated in an impingement-fin-film cooling configuration. It was shown that increasing the blowing ratio increased film cooling effectiveness for cases with and without the blockages. Furthermore, results for low blowing ratios show a slight deterioration in effectiveness for blockages located at the trailing edge of the hole exit. Results also show a slight improvement in effectiveness for the other locations. In addition, it was shown that for high blowing ratios the counter rotating vortex pair were mitigated for blockages located at the leading edges of the hole inlet and exit, thus resulting in a slight improvement in effectiveness.

Huang et al. [18] experimentally studied the effect of partial blockages at the leading and trailing edges of the hole on film cooling effectiveness. Results show that the presence of a hole blockage located at the hole exit and positioned at the leading edge increased the film cooling effectiveness by $20 \%$ between $2<\frac{x}{D}<12$, thus validating the findings of Pan et al [16]. It also was found that a hole blockage located at the hole exit and positioned at the trailing edge cause a film cooling lateral effectiveness deterioration of about $50 \%$. Bunker [19] performed and experimental study on the effect of hole partial blockage caused by protective layers such as TBC on film cooling effectiveness for round and diffusion shaped holes. The protective layers on the plates were found to reduce the area of the round holes and diffusion holes by $30 \%$ and $15 \%$ respectively. Furthermore, results show that the centerline adiabatic effectiveness for the samples containing the coatings are reduced by $50 \%$ and $30 \%$ for the round and diffusion holes respectively compared to the samples without the coatings. The diffusion holes were found to mitigate the detrimental 
effect of the blockages and allowed the film cooling effectiveness to recover to a certain degree to the values found by samples without the blockages.

Wang et al [20] performed numerical simulations on the effects of TBC distortions near the hole exit by modelling the deposition with a bump with a semi elliptical profile extending laterally through the computational domain. The width and the height of the deposition where varied in order to investigate their influence on film cooling effectiveness. It was found that the presence of the deposition deteriorated the cooling effectiveness by a factor of two for distances beyond eight diameters from the hole exit. Results show that larger deposition widths with lower heights causes the least deteriorations to film cooling performance.

Rozati et al. [21] conducted large eddy numerical simulations on the effect of syngas ash particle size on depositions and erosions caused on a film cooled leading edge. Five sizes of 1, 3, 5, 7 and $10 \mu \mathrm{m}$ were investigated at various blowing ratios. Results reveal that the majority of $1 \mu \mathrm{m}$ particles follow the flow streamlines and only a few reaches the blade surface. In addition, it was seen that $90 \%$ of the $10 \mu \mathrm{m}$ particles impinge and deposit on the blade surface and coolant hole. Furthermore, it was shown that as the blowing ratio is increased, the 1 and $3 \mu \mathrm{m}$ particle depositions increase as well. However, the contrary is true for the 5,7 and $10 \mu \mathrm{m}$.

Walsh et al. [22] conducted experimental investigations on the effect of sand particle ingestions on the blockage of film cooling holes. Important parameters influencing hole blockage such as pressure ratio, sand amount, sand distribution, hole quantity and metal temperatures were studied. It was found that increasing the pressure ratio increases the flow, and particle, velocity and thus reduces the likelihood of particle adhesion and hole blockage. In addition, results reveal that increasing sand amounts increases hole blockages. Furthermore, results show that high metal or surface temperature increases sand particle adhesion to the surface.

Lawson and Thole [23] experimentally simulated particle deposition using a low melting temperature wax particles injected into the mainstream at various momentum flux ratios. It was found that in the near wake region of the jet, only large molten particles were found to adhere to the surface and resist changes in trajectory. Moreover, as depositions occurred, an increase in both particle size and depositions was witnessed suggesting that new deposits adhered to existing ones.

Lawson and Thole [24] experimentally investigated the effects of simulated multiphase particle deposition using a low melting temperature wax similar to the one used in [23] on endwall film cooling. Wax was supplied using an atomizing spray nozzle in order to deliver solid and molten particulate matter. Results reveal that the vane stagnation area contains a dense amount of depositions compared to other areas. It was found that a buildup of deposition deteriorated in adiabatic film cooling effectiveness by $30 \%$ and that results were dependent on the location of the cooling holes with respect to the vane. 
Lawson and Thole [25] experimentally studied the effects of simulated multiphase particle deposition on endwall film cooling hole located in transverse trenches. The investigation was aimed at determining how the beneficial effect of trenches were affected by these depositions. It was shown that particle depositions deteriorated film cooling effectiveness by as much as $15 \%$ compared to $30 \%$ for cases without the trenches. Furthermore, results reveal that trenches with a depth of $0.8 \mathrm{D}$ had the highest effectiveness compared to other cases.

Lawson et al. [26] experimentally studied the effects of simulated multiphase particle deposition on a showerhead with staggered film cooling holes. Similarly, to other findings for other locations, an increase in blowing ratio resulted in a decrease in particle depositions. However, the detrimental effect that the particle deposits, at higher blowing ratios, have on film cooling effectiveness is more significant than the ones present at lower blowing ratios. Results show that film cooling effectiveness deteriorations causes by particle depositions on a showerhead reached as high as 36\% for a blowing ratio of 1 .

Ai et al. [27] performed experimental studies on depositions of ground coal ash near film cooling holes on a high pressure turbine vane. Cylindrical and diffusion shaped holes with and without TBC coating applied were studied. Deposition patterns for cylindrical holes show an accumulation area between the film holes and low deposition was found in the coolant path. This pattern was seen to be more visible as blowing ratios increased and resulted in higher temperature in the areas between the coolant paths as depositions augmented. It was found for the shaped holes that ash deposition was minimal to non present downstream due to the coolant distribution. Moreover, close hole spacing, low pitch to diameter ratio, of cylindrical hole were found to give similar results to shaped holes.

Ai et al. [28] experimentally investigated the effect of hole spacing, or pitch to diameter ratio, on the deposition of coal flyash near cooling holes with and without surface coating. Results reveal that for the same hole spacing, the surface containing the coating captured more deposits than the one without TBC due to high surface temperatures experienced which was also observed in [27]. It was found that both hole spacing and blowing ratio have an influence on surface deposition. Close hole spacing and high blowing ratios were found to cause the least coal ash depositions.

Cardwell et al. [29] performed experimental studies on the effect of sand blocking within impingement and film cooling holes. Four different liner configurations were investigated where parameters such as pressure ratio, sand quantity, metal and sand temperatures were used to assess their performance. Results reveal that an increase in pressure ratio across the liners caused an increase in hole blockages. It was found that with the impingement and film cooling configuration, sand particles where found to pass through the film cooling holes much easier because they get broken up as they get impinged on the upstream side of the film cooling plate. Additionally, it was found that for high metal and sand temperatures the sand became sticky and deposits and blockages where more apparent. Furthermore, more blockage was observed for liner 
configurations where the impingement holes overlapped the film cooling holes compared to the staggered configurations.

In order to reduce sand blockage, Land et al. [30] considered using a double-wall cooling design as used in [29]. Results revealed that hole blockages were mainly caused by large sand particles, greater than $150 \mu m$, and it was found that a spacing of two to four impingement diameters between the impingement and film cooling plates results in the least particle blockage. For large plate spacing, the sand particles do not have enough momentum to break up into smaller particles when impinged. For small plate spacing, the crossflow between the holes has enough influence on the sand particles to interfere in the impingement process.

Research on the effect of imperfections formed in the manufacturing process, such as laser percussion drilling, is needed in order to know if the flow characteristics affected by hole imperfections are behaving differently than originally intended or not. One of the first papers addressing the influence of laser drilling imperfections on film cooling performances was written by Jovanovic et al [9,31]. A transverse jet in a crossflow with an imperfection simulated by a half torus placed inside the hole at three distinct positions was used. It was found that the imperfection changed the flow field around the hole exit influencing the film cooling performance in the vicinity of the hole. The imperfection caused the jet to accelerate and penetrate deeper into the crossflow deteriorating the film cooling effectiveness. This phenomenon was more pronounced for the imperfection located nearest to the hole exit. It was found that unlike the perfect case, holes containing the imperfection are dependent on the blowing ratio. For blowing ratios greater than 0.5 , the penetration of the crossflow was found to be significant causing the decreases in film cooling effectiveness.

Nemdili et al [32] performed a numerical investigation of jet-crossflow interaction with the hole imperfection matching the conditions of Jovanovic et al [9]. The influence of the imperfection size was investigated and it was found that when the obstruction covers over $50 \%$ of the hole area, a dramatic decrease in film cooling performance is observed. Effectiveness contours and velocity vector plots reveal that when the size of the imperfection increases, the center of the counter rotating vortex pair (CRVP) is seen to be farther from the plate allowing some of the hot gases to enter the injection hole. Moreover, the effect of the position of the imperfection on the film cooling performance was investigated. It was observed that when the imperfection was located at the hole exit, film cooling effectiveness decreased. However, as the imperfection is positioned away from the hole exit, the jet recovers and the film cooling performance is unaffected.

Jovanovic et al [33] conducted an experimental investigation of the effect of hole imperfections when the hole is inclined at an angle. Three blowing ratios were tested while the position of the imperfection was held constant. The imperfection was found to decrease the film cooling effectiveness at low blowing ratios whereas at higher blowing ratios the effectiveness is enhanced in the vicinity of the hole. Moreover, 
additional toroidal vortex warps form above the imperfection disturbing the flow flied at the hole exit. Jovanovic et al [34] studied the effect of turbulence intensity with the presence of the imperfection along with its position on film cooling performance. It was observed that the turbulence intensity did not have an effect on the adiabatic effectiveness except for when the imperfection is placed near the hole exit (0.2D) at a blowing ratio of 0.7 . The equivalent cooled surface was found to be reduced by about $10 \%$. In addition, it was found that the best cooling effectiveness for imperfections positioned at $1.2 \mathrm{D}$ and $2.5 \mathrm{D}$ is achieved at a blowing ratio of 0.4 .

Cerantola and Birk [35] numerically investigated as-drilled hole geometries captured using 3D X-RAY tomography which was compared with cylindrical and conical nozzled holes. The cylindrical hole provided better coolant coverage than the other two cases. The as-drilled hole had better laterally-averaged effectiveness than the conical nozzle for $\mathrm{x} / \mathrm{D}$ less than 5 due to additional turbulence generated within the hole.

Bharathkumar et al [36] performed a 2D numerical investigation of the effect of a hole imperfection on film cooling. The geometry consisted of a hole inclined at $35^{\circ}$ with a length to diameter ratio of 10 and no plenum was used. It was found that the presence of the imperfection deteriorated the film cooling performance for a blowing ratio of 0.75 which is caused by a flow separation occurring above the imperfection. This flow separation was found to cause turbulence and an increase in mixing between the hot gases and coolant which leads to decreased protection.

\section{Flow Structure}

In order to interpret film cooling or jet in crossflow results, it is necessary to have an understand on main flow physics and structures formed in those situations. Fric and Roshko [37] performed experimental investigations in order to identify the main or dominant vortical structures developed for jet in crossflows. The four main vortical structures identified are the jet shear-layer vortices, the counter-rotating vortex pair (CRVP), horseshoe vortices and the wake vortices as seen in Figure 8. The shear-layer vortices were found to be near-field structures that are shaped in the form of consecutive vortex rings. The counter-rotating vortex pair is the main vortical structure responsible for mixing between the jet and crossflow and is studied extensively to either enhance or prevent mixing. For film cooling applications, research is being done in order to suppress the CRVP so that the coolant jet film lays as much as possible on top the surface without mixing with the hot freestream gases. The horseshoe vortices were found to be a result of crossflow boundary-layer separation upstream of the jet. Finally, the wake vortices are vertical structures located downstream of the jet exit between the surface and jet core. It was found that their formation is due to the crossflow boundary layer separation. 


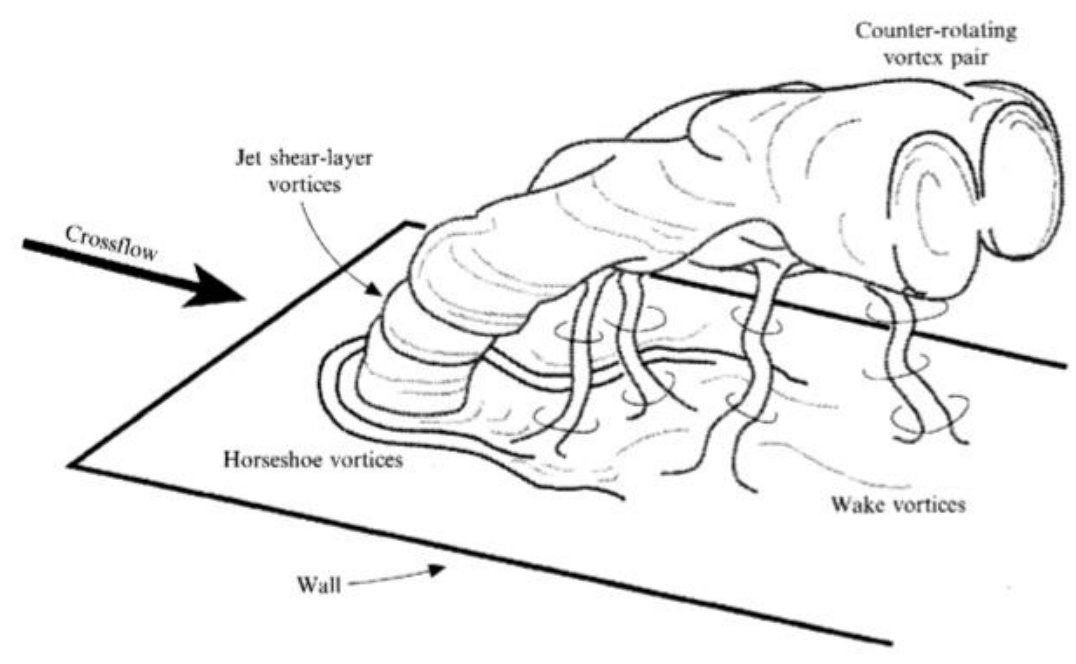

Figure 8. Jet in crossflow structure [37]

Haven et al. [38] have experimentally investigated the effect of hole geometry and aspect ratio on the kidney, or CRVP, and anti-kidney vortices. Six hole geometries with various aspect ratio, where high aspect ratios correspond to wider hole exit in the lateral direction, were investigated. Results reveal that hole geometries and aspect ratio have a strong influence on jet lift off. It was shown that holes with high aspect ratios inhibited jet lift even for high blowing ratios as seen in Figure 9.

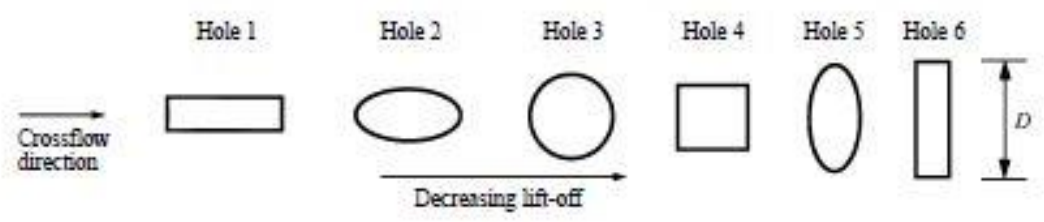

Figure 9. Trend of jet lift off with hole geometry [38]

Ely and Jubran [39] have conducted numerical investigations on the effect of sister hole location on active film cooling flow control and finding the optimal hole configuration. The sister hole concept consists of place smaller diameter holes located on either sides of the main or primary cooling hole in order to supress the counter rotating vortex pair thus inhibiting jet lift off. Results show that placing the sister holes slightly upstream of the main hole improves film cooling effectiveness in the near hole region while sister holes located slightly upstream improves the effectiveness downstream of the hole.

Ely and Jubran [40] performed computational simulations on the effect of short sister holes on film cooling effectiveness. It was found that compared to long holes, short holes generated similar but less coherent flow 
field due to the underdeveloped flow emanated from the holes. Nonetheless, sister holes provide great improvements to film cooling effectiveness compared to standard holes.

Siavash and Jubran [41] numerically investigated the effect of three novel sister shaped single-holes on film cooling performance by comparing them to a standard hole. In this instance, the sister holes were emanating from the main holes instead of the plenum. This was done in order to further increase the coolant's adhesion to the plate and reduce the amount of the coolant compared to discrete sister holes. Results reveal that the sister hole configuration outperformed both the single and shaped holes. For instance, at a blowing ratio of 1 , the sister holes emerging downstream of the main hole improved the laterally averaged effectiveness by $110 \%$ compared to the forward diffused shaped hole.

Siavash and Jubran [42] computationally evaluated the effect of the sister shaped single-holes configurations developed in [41] on turbine leading edge film cooling performance. It has been observed that the laterally averaged effectiveness was improved, particularly on the suction side, for the sister hole configurations. Moreover, the presence of the sister holes caused less mixing to occur and resulted in the coolant to adhere more to the surface.

Siavash and Jubran [43] numerically investigated the film cooling performance of main circular exit shaped hole with the influence of discrete sister holes. The effects of the circular exit shaped hole were monitored against the elliptic exit shaped hole as a baseline. It important to note that in order to get a circular exit shaped hole the profile of the pipe need to be elliptic. Results indicate that the circular exit shaped hole outperformed, especially at high blowing ratios, the elliptic exit shaped hole in centerline and laterally averaged effectiveness. By adding sister holes to the circular exit shaped hole, the CRVP were significantly weakened thus a resulting increased in film cooling performance occurred.

Rigby [44] performed computational simulations on the effect of a delta vortex generator located downstream of the cooling holes. It was found that the delta vortex generator caused the film cooling effectiveness to improve significantly. The generator was observed to eliminate the lift off effect of the CRVP and create a downwash pair spreading along the surface. This was done by separating the CRVP and avoiding the pair to interact with each other.

In an attempt to increase film cooling effectiveness and reduce coolant amount, Kusterer et al. [45] invented the Double-Jet Film-Cooling (DJFC) technology. In order to achieve this feat, the DJFC technology consists of establishing an anti-vortex pair by combining two jets emerging from two discrete holes with compound angles one located after the other. Numerical results reveal that for low blowing ratios, better film cooling performance is achieved if the holes are located slightly out of the centerline. This configuration provides significantly higher film cooling effectiveness lasting far downstream, $x>30 D$. Furthermore, it was found that placing the holes farther from the centerline causes adverse effects in effectiveness because the generated anti-kidney vortices enables the hot gases to reach the surface. 


\section{Parametric Study}

Yuen and Martinez-Botas [46] performed experimental studies investigating the effect of hole inclination angle on film cooling effectiveness over a flat plate. Three hole inclination angles $\left(30^{\circ}, 60^{\circ}\right.$ and $\left.90^{\circ}\right)$ were studied for blowing ratios ranging from 0.33 to 2 . It was observed that the $30^{\circ}$ hole inclination enabled superior film cooling performance for the near and far field regions. The $60^{\circ}$ provided better effectiveness in the intermediate region.

Prasad et al [47] performed numerical simulations investigating the effect of hole injection angle on the film cooling performance over a flat plate. The hole inclination angle of $30^{\circ}, 60^{\circ}$ and $90^{\circ}$ were studied at six blowing ratios similar to [46]. Results revealed that the hole inclination angle of $30^{\circ}$ outperformed the other two angles in film cooling effectiveness. Furthermore, it was found that increasing the blowing ratio causes an increase in vortices intensity thus deteriorating effectiveness.

Yavuzkurt and Habte [48] investigated the effect of computational mesh configurations on the performance of two-equation turbulence models in predicting film cooling effectiveness. The k-epsilon models, standard, RNG and realizable, and the standard k-omega model were investigated. Results show that grid quality has a lot of influence on the data. For instance, results from non-conformal mesh configurations with tetrahedral cells show inconsistencies with effectiveness data. Much better agreement with experiment results was obtained for conformal mesh configurations with hexahedral cells. All turbulence models provided good predictions for a blowing ratio of 0.5 .

Harisson and Bogard [49] compared three RANS turbulence models in order to evaluate their performance in predicting film cooling effectiveness over a flat plate. The turbulence models evaluated are the k-epsilon, k-omega and RSM models. Results show that the turbulence model that best predicted the laterally averaged effectiveness was the k-omega model. However, this model was the least accurate in predicting the centerline film cooling effectiveness. Moreover, it was found that the turbulence model that best predicted the centerline effectiveness was the k-epsilon model. However, this model was the least accurate in predicting the laterally averaged effectiveness.

Tao et al [50] performed numerical and experimental studies in order to investigate the performance of three turbulence models in predicting film cooling effectiveness over a rotating blade. The turbulence models evaluated were the standard k-epsilon model, the standard k-omega model and the SST k-omega. It was shown that the rotating speed is the parameter that has the most influence on film cooling effectiveness and that results can differ significantly from the pressure and suction sides. Furthermore, results show that all turbulence models over predicted the film cooling effectiveness with the standard kepsilon giving the least accurate prediction.

Keimasi and Taeibi-Rahni [51] numerically simulated normal square jets in a cross flow using the standard k-epsilon and SST k-omega turbulence models. Numerical results show good agreement in predicting the 
velocity vector field with the experimental data compared to previous numerical investigations. Even though the velocity vector field results agree, the contrary was true in predicting the turbulent kinetic energy results.

Zuniga and Kapat [52] experimentally investigated the effect of hole pitch to diameter ratio on the film cooling performance of standard and diffusion shaped holes located in trenches. The hole pitch to diameter ratio studied range from 4 to 12 at blowing ratios ranging from 0.5 to 2 . The results were compared the cases without the trenches in order to monitor the effects. It is important to note that decreasing the number of holes is desirable in order to decreasing the amount of coolant used. However, decreasing the number of holes and spacing them out, thus increasing pitch to diameter ratio, decreases film coverage across the surface. Results show that embedding the film cooling holes inside trenches and increasing pitch to diameter ratio can maintain the same cooling performance while reducing the number of holes by half. However, placing shaped holes in trenches was found to adversely affect effectiveness.

Zhou et al. [53] performed computational simulations investigating the effects of hole pitch to diameter ratio on film cooling performance on a liner configuration similar to [29]. The pitch to diameter ratios of 3 , 4, 5 and 6 were chosen for the film cooling holes whereas 4, 6, 8 and 10 were chosen for the impingement holes. For this configuration, results reveal that an overall improvement in effectiveness occurred for lower film cooling hole pitch to diameter ratios. It was also observed that decreasing the pitch to diameter ratio below three can lead to blockages in the film cooling holes.

Laroch [54] numerically investigated the influence of freestream turbulence intensity on film cooling effectiveness over a flat plate. Blowing ratios of 0.7 and 1.15 and freestream turbulence intensity of $0.9 \%$ and $12 \%$ were investigated. Results show that an increase in freestream turbulence intensity adversely affects film cooling performance for all blowing ratios investigated. 


\section{PROBLEM STATEMENT}

This chapter presents the steps carried out to investigate the effect of hole imperfection, due to laser percussion drilling, on film cooling performance. The geometry and measures of the computational domain with and without the imperfection are first considered. A thorough discussion of the flow parameters such as blowing ratio, density ratio, Reynolds number and turbulence intensity is then deliberated. The boundary conditions imposed on the computational domain and the grid sensitivity test will then be discussed. Finally, this chapter will conclude with a discussion of how convergence was considered. Figure 10 summarizes the outline of this chapter.

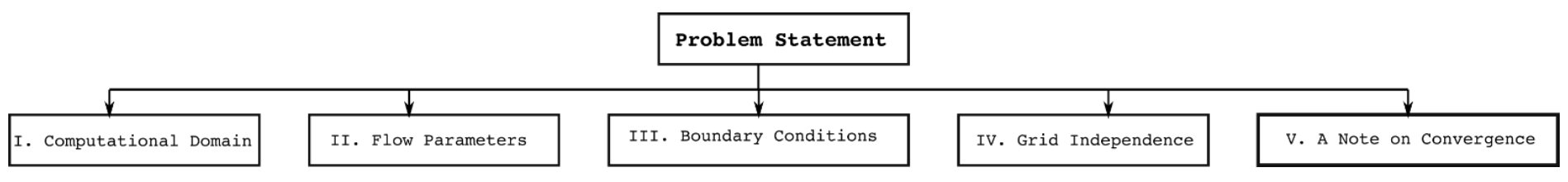

Figure 10. Problem Statement Outline 


\section{Computational Domain}

The geometry used in this investigation is modeled according to the experimental setup of Jovanovic et al. [33]. The dimensions of the computational domain were chosen in order to scale the Reynolds' numbers based on the hole diameter and on the distance from the plate leading edge to the hole leading edge with the setup. This was done because the low freestream velocity of $0.2 \mathrm{~m} / \mathrm{s}$ used in the experiment would make it difficult to achieve desired y+ values. Therefore, a freestream velocity of $20 \mathrm{~m} / \mathrm{s}$ was deemed high enough to be able to achieve low $y+$ values required for various turbulence models. As shown in Figure 11, the computational domain is extended for 28.5D and 40D from the leading and trailing edges of the injection hole, respectively. The cylindrical hole has a diameter of $0.57 \mathrm{~mm}$ with a pitch-to-diameter ratio of 3D, a length-to-diameter ratio of 10 and an inclination angle of $35^{\circ}$. Since the hole is long (L/D>5) [55], the flow in the pipe is considered to be fully developed. Therefore, the addition of a plenum is deemed unnecessary for this case resulting in a reduction in computational cost.

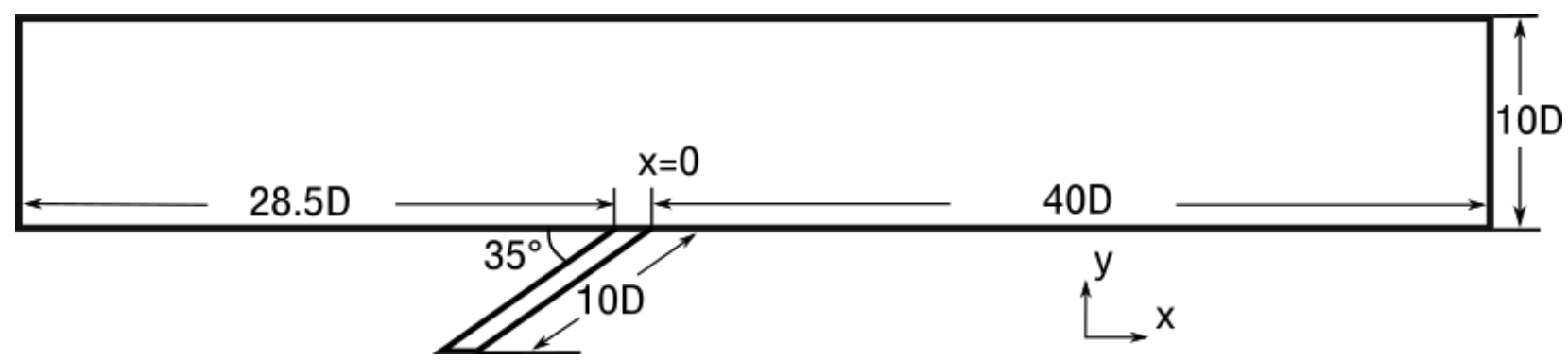

Figure 11. Computational Domain Geometry

The hole imperfection was modeled as an inner torus located 1D from the hole exit leading edge as can be seen in Figure 12. The radius of the inner torus is set to $0.075 \mathrm{~mm}$ reducing the hole area by $26 \%$.

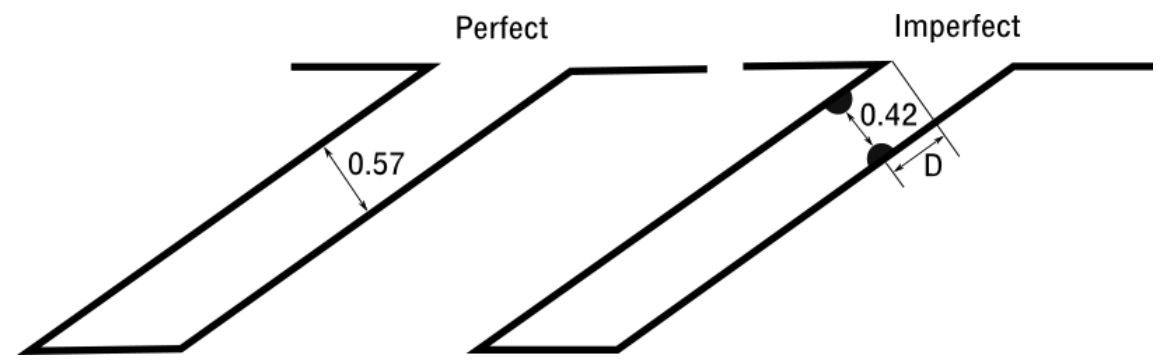

Figure 12. Geometry of the Perfect and Imperfect Hole 


\section{Numerical Method}

The simulations were performed using ANSYS FLUENT 17.1 which solves the RANS equations using finite volume discretization. A pressure-based type solver was chosen along with an absolute velocity formulation at steady state. The incompressible flow simulations were performed with water as the working fluid with the density set as $998.2 \mathrm{~kg} / \mathrm{m}^{3}$, the specific heat as $4182 \mathrm{~J} / \mathrm{kgK}$, the thermal conductivity as 0.6 $\mathrm{W} / \mathrm{mK}$ and the viscosity as $0.001003 \mathrm{~kg} / \mathrm{ms}$. The SIMPLEC scheme was chosen to solve the pressurevelocity coupling. The second order upwind scheme is used to solve the momentum, turbulent kinetic energy, turbulent dissipation rate and energy equations. The second order and least squares cell based schemes were used for the pressure and gradient respectively. High order term relaxation was also considered. The solutions were considered to have converged when the normalized residuals reached $10^{-5}$ for all variables and $10^{-7}$ for the energy equation. The laterally average adiabatic effectiveness was computed from the predicted wall temperature as

$$
\bar{\eta}=\frac{1}{D} \int_{-0.5 D}^{0.5 D} \eta d z
$$

The adiabatic film cooling effectiveness in Eq. (5) takes the average effectiveness along the lateral ( $\mathrm{Z}$ axis) going from $-0.5 \mathrm{D}$ to $0.5 \mathrm{D}$ of the centerline.

\section{Flow Parameters}

In all the numerical simulations performed in this thesis, the blowing ratios of $0.45,0.9$ and 1.25 are investigated at a density ratio of 1 . The Reynolds number based on the distance from the leading edge of the plate to the hole leading edge is $R e_{x}=3.2 \times 10^{5}$ resulting in a distance of $16.25 \mathrm{~mm}$ for a freestream velocity of $20 \mathrm{~m} / \mathrm{s}$. Table 2 displays the Reynolds number based on the hole diameter for each blowing ratio along with the corresponding coolant jet velocity for both the perfect $(\mathrm{P})$ and imperfect or inner torus cases (I).

Table 2 Coolant jet velocity

\begin{tabular}{|c|c|c|c|c|}
\hline & Case & $\mathrm{V}_{\text {jet }}(\mathrm{m} / \mathrm{s})$ & Blowing Ratio-M & $\mathrm{Re}_{\mathrm{D}} \times 10^{3}$ \\
\hline 1 & $\mathrm{P} 1, \mathrm{I} 1$ & 9 & 0.45 & 5.13 \\
\hline 2 & $\mathrm{P} 2, \mathrm{I} 2$ & 18 & 0.90 & 10.26 \\
\hline 3 & $\mathrm{P} 3, \mathrm{I} 3$ & 25 & 1.25 & 14.25 \\
\hline
\end{tabular}


In order to properly model the hole inlet conditions, the turbulence intensity must be carefully calculated. Russo and Basse [56] have provided a scaling formula for incompressible flow turbulence intensity based on measurements, modelling and computational fluid dynamics as

$$
I_{\text {Incompressible CFD,pipe axis }}=0.0853 \times R e^{-0.0727}
$$

Table 3 displays the hole inlet turbulence intensities for each Reynolds number corresponding to the three blowing ratios.

Table 3 Hole inlet turbulence intensity

\begin{tabular}{|c|c|c|c|c|}
\hline & Case & $\begin{array}{c}\text { Turbulence } \\
\text { Intensity (\%) }\end{array}$ & Blowing Ratio-M & $\mathrm{Re}_{\mathrm{D}} \times 10^{3}$ \\
\hline 1 & $\mathrm{P} 1, \mathrm{I} 1$ & 0.0458 & 0.45 & 5.13 \\
\hline 2 & $\mathrm{P} 2, \mathrm{I} 2$ & 0.0436 & 0.90 & 10.26 \\
\hline 3 & $\mathrm{P} 3, \mathrm{I} 3$ & 0.0426 & 1.25 & 14.25 \\
\hline
\end{tabular}

\section{$\underline{\text { Boundary Conditions }}$}

The boundary conditions imposed on the computational domain are as shown in Figure 13. The current simulations are carried out where the jet temperature at the hole inlet is set at $26^{\circ} \mathrm{C}$ and the freestream temperature at $20^{\circ} \mathrm{C}$. This is in accordance to the experimental setup of Jovanovic et al [33]. The turbulence intensity and hydraulic diameter of the freestream velocity inlet and pressure outlet were set to $1 \%$ and $2.63 \mathrm{~mm}$, respectively.

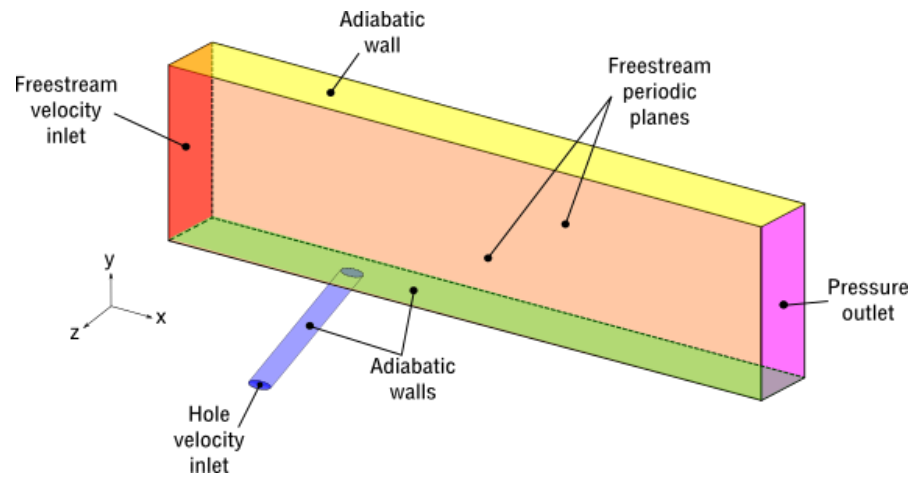

Figure 13. Computational Domain Boundary Conditions

\section{Grid Independence}

The mesh configurations for the computational domain were generated by ICEM CFD. As shown in Figure 14 , the hexahedral cells were carefully distributed across the computational domain where they were highly 
refined near the hole exit and the walls. An O-grid was used in order to better capture the flow physics in the vicinity of the hole exit. A $\mathrm{y}^{+}$value near 1 was achieved by varying the distance and concentration of the cells above the plate. This value was chosen because it is a requirement of the turbulence models chosen to perform the simulations. Figure 15 a shows the wall $\mathrm{y}^{+}$value variation downstream of the hole exit along the centerline at a blowing ratio of 0.45 and density ratio of 1 .

Three mesh configurations consisting of 521900, 1075130 and 1506490 elements have been built in order to conduct a grid sensitivity test. As shown in Figure 15b, the solution at a blowing ratio of 0.9 is shown to reach grid independence at 1075130 elements. Therefore, the second mesh configuration is used for validation in the next section.

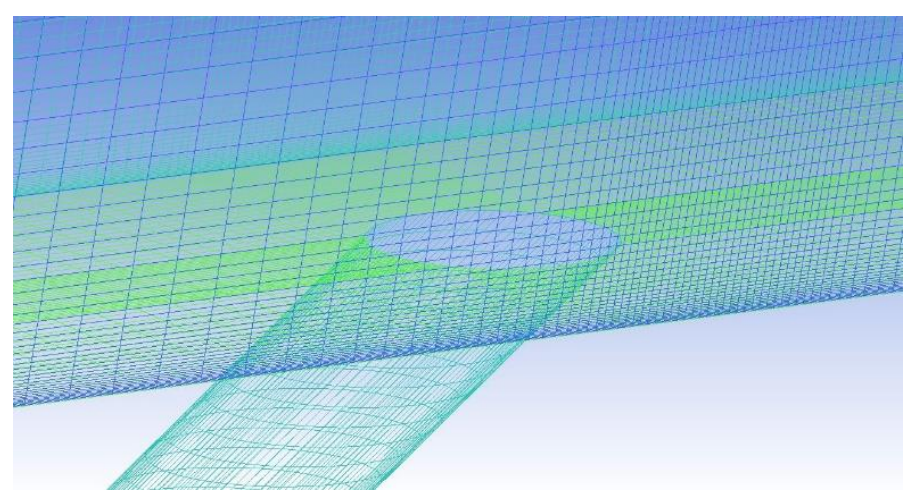

Figure 14. Mesh Configuration Close Up

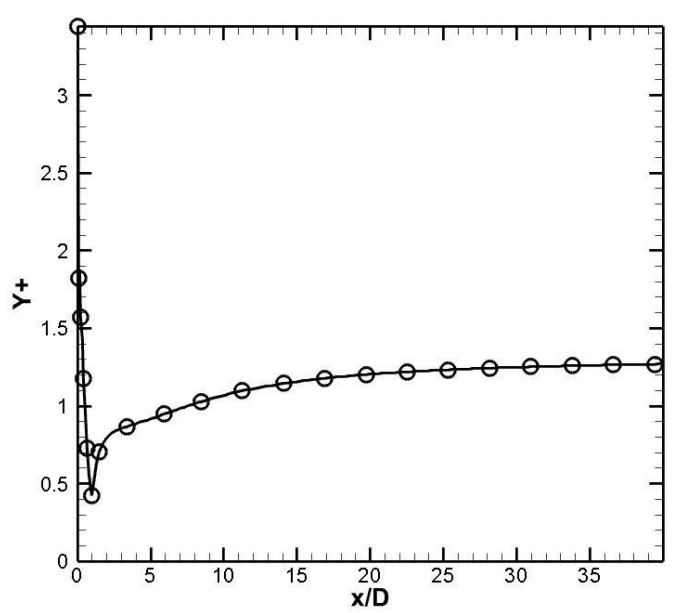

a) Y+ value

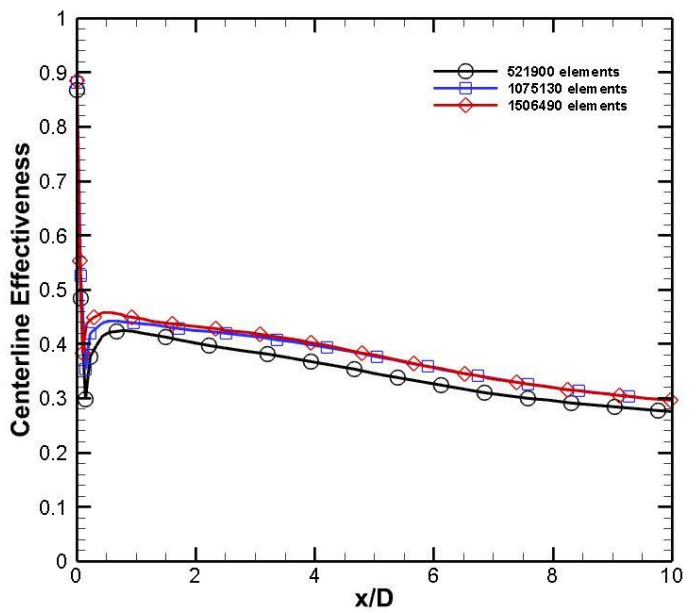

b) Grid Sensitivity

Figure 15. Mesh Information 


\section{$\underline{\text { A Note on Convergence }}$}

When a computational simulation has reach a pre-set criterion for determining convergence, residuals in this case, it does not guarantee the convergence of the solution. While performing numerical simulations for this thesis, the residual for the continuity equation reached the desired value last. Therefore, making sure the residual for the continuity equation automatically satisfies the residual conditions of the other equations. In order to guarantee the convergence of the solution, the centerline effectiveness at various residual values was compared and the solution was considered to be converged when the no more changes are observed, see Figure 16.

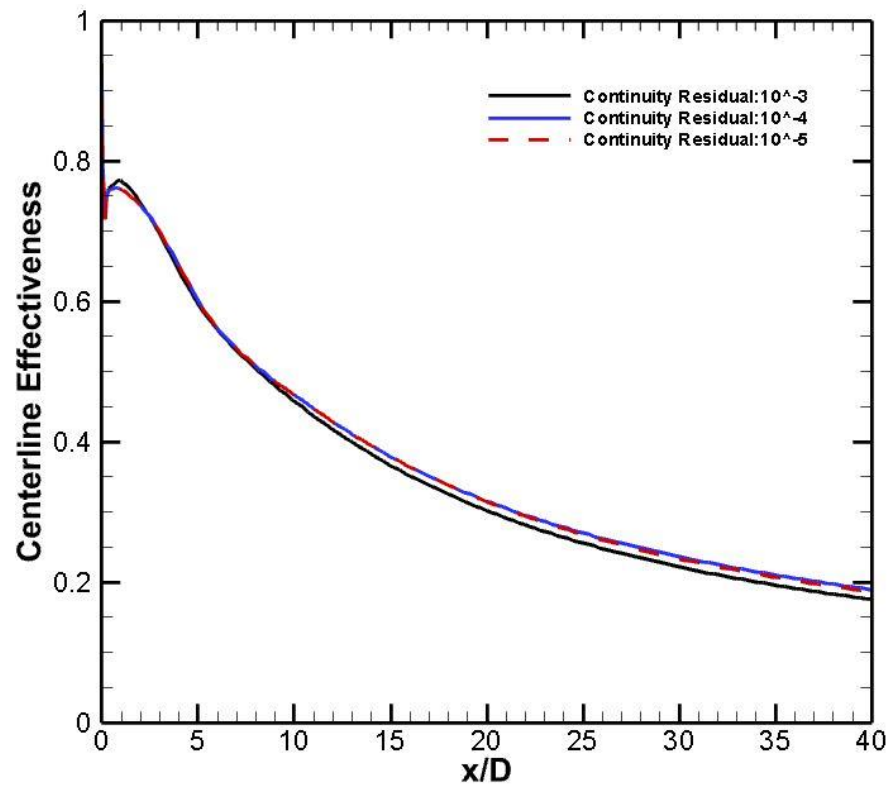

Figure 16. Convergence 


\section{RESULTS AND DISCUSSION}

Now that the computational domain, flow parameters, boundary conditions and grid independence have been set, this chapter presents the results obtained from the numerical simulations. However, before moving forward in the investigation of the effect of imperfection, it is necessary to validate the results and find out which turbulence model is capable of providing accurate predictions. After the validation is complete, the effects of hole imperfection position, hole inclination angle and imperfection shape on film cooling performance is investigated. Figure 17 summarizes the outline of this chapter.

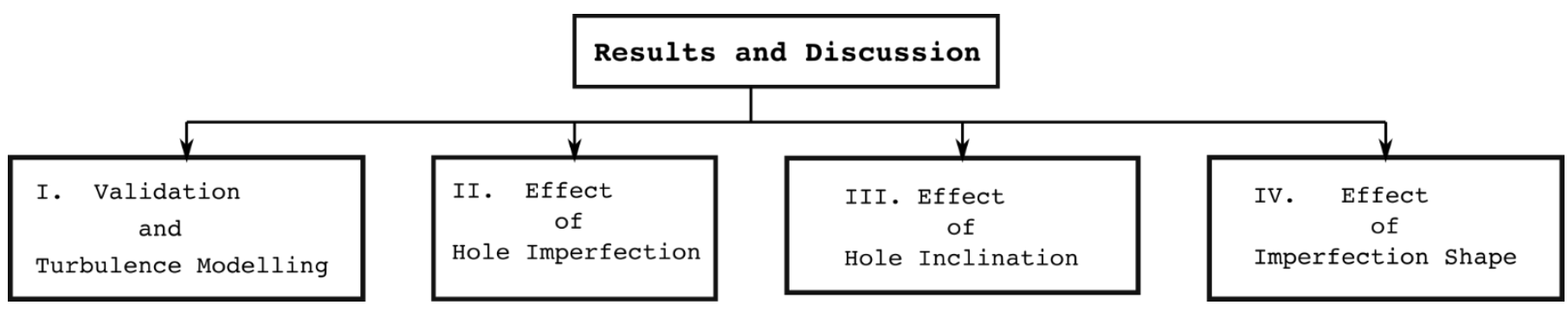

Figure 17. Results and Discussion Outline 


\section{$\underline{\text { Validation and Turbulence Modelling }}$}

In order to proceed to the results, the simulated solutions are validated against the experimental data provided by Jovanovic et al [33].

\section{Governing equations}

In the present investigation, the working fluid is considered to be incompressible, Newtonian with temperature dependent fluid properties. Hence, the Reynolds averaged Navier Stokes equations (RANS) along with the energy equations are as follows:

$$
\begin{gathered}
\frac{\partial U_{i}}{\partial x_{j}}=0 \\
\rho U_{i} \frac{\partial U_{j}}{\partial x_{i}}=-\frac{\partial p}{\partial x_{j}}+\mu \frac{\partial^{2} U_{j}}{\partial x_{i} \partial x_{i}}+\frac{\partial}{\partial x_{i}}\left[\rho \overline{u_{\imath} u_{j}}\right] \\
U_{i} \frac{\partial \Theta}{\partial x_{i}}=\alpha \frac{\partial^{2} \Theta}{\partial x_{i} \partial x_{i}}-\frac{\partial}{\partial i}\left[\overline{u_{\imath} \Theta}\right]
\end{gathered}
$$

The evaluation of turbulence models was evaluated by two mesh configurations where one has a y+ value above 30 and the other with a $y+$ value of 1 . All turbulence models coupled with wall functions were associated with the mesh configuration have a y+ value of 30. The standard, RNG and realizable k-epsilon and the Reynolds Stress turbulence models with the standard, scalable, non-equilibrium wall functions and enhanced wall treatment were investigated. Moreover, the standard, BSL and SST k-omega turbulence models were also investigated. All the results are provided in Appendix A for both the perfect and imperfect cases. In this paper, the SST k- $\omega$ and realizable k- $\varepsilon$ (coupled with the enhanced wall treatment) turbulence models will be used as they provide the best predictions of effectiveness compared to other models. A brief description of each is provided next.

\section{SST k- $\omega$ Model}

The SST k-omega turbulence model is a variant of the k- $\omega$ model. It combines the original Wilcox model for use near walls with the standard k-epsilon model away from walls using a blending function. It limits the turbulent viscosity in order to guarantee that $\tau_{T} \sim k$. The SST k-omega model is suitable for complex boundary layer flows under adverse pressure gradient and separation. This model is known to provide more 
accurate predictions of flow separation than other RANS models. The SST k-omega model is as follows $[57,58]$ :

The eddy viscosity,

$$
\mu_{T}=\rho k / \omega
$$

Turbulence kinetic energy,

$$
\rho \frac{\partial k}{\partial t}+\rho U_{j} \frac{\partial k}{\partial x_{j}}=\tau_{i j} \frac{\partial U_{i}}{\partial x_{j}}-\beta^{*} \rho k \omega+\frac{\partial}{\partial x_{j}}\left[\left(\mu+\sigma^{*} \mu_{T}\right) \frac{\partial k}{\partial x_{j}}\right]
$$

Dissipation rate,

$$
\rho \frac{\partial \omega}{\partial t}+\rho U_{j} \frac{\partial \omega}{\partial x_{j}}=\alpha \frac{\omega}{k} \tau_{i j} \frac{\partial U_{i}}{\partial x_{j}}-\beta \rho \omega^{2} \quad+\frac{\partial}{\partial x_{j}}\left[\left(\mu+\sigma \mu_{T}\right) \frac{\partial \omega}{\partial x_{j}}\right]
$$

Closure coefficients,

$$
\begin{gathered}
\alpha=1.44 \quad \beta=1.92 \quad \beta^{*}=0.09 \quad \sigma=1.3 \\
\sigma^{*}=1.0
\end{gathered}
$$

Auxiliary relations,

$$
\varepsilon=\beta^{*} \omega k \quad l=k^{1 / 2} / \omega
$$

\section{Realizable k-E Model}

The realizable k-epsilon turbulence model is a variant of the k-epsilon model. This model allows certain mathematical constraints to be obeyed which improves its performance. The realizable k-epsilon model is suitable for complex shear flows involving rapid strain, moderate swirl, vortices and locally transitional flows. Examples of locally transitional flows are boundary layer separation, massive separation, vortex shedding behind bluff bodies, stall in wide-angle diffusers and room ventilation. This model is promising for film cooling applications due to its ability to predict moderate swirls and vortices which are present in film cooling. The transport equation for the turbulence kinetic energy is the same as the one for the standard $\mathrm{k}$-epsilon turbulence model. The realizable k-epsilon model is as follows [57, 58]:

The eddy viscosity,

$$
\mu_{T}=\rho C_{\mu} k^{2} / \epsilon
$$


Turbulence kinetic energy,

$$
\rho \frac{\partial k}{\partial t}+\rho U_{j} \frac{\partial k}{\partial x_{j}}=\tau_{i j} \frac{\partial U_{i}}{\partial x_{j}}-\rho \epsilon+\frac{\partial}{\partial x_{j}}\left[\left(\mu+\mu_{T} / \sigma_{k}\right) \frac{\partial k}{\partial x_{j}}\right]
$$

Dissipation rate,

$$
U_{i} \frac{\partial k}{\partial x_{i}}=C_{1} S_{\epsilon}-C_{2} \frac{\epsilon^{2}}{k+\sqrt{\mu \epsilon}}+\frac{\partial}{\partial x_{j}}\left[\left(\mu+\mu_{T} / \sigma_{\epsilon}\right) \frac{\partial k}{\partial x_{j}}\right]
$$

Closure coefficients,

$$
\begin{gathered}
C_{1}=\max \left[0.43, \frac{\eta}{\eta+5}\right], \quad \eta=S \frac{k}{\epsilon}, \quad S=\sqrt{2 S_{i j} S_{i j}} \\
S_{i j}=\frac{1}{2}\left(\frac{\partial U_{j}}{\partial x_{i}}+\frac{\partial U_{i}}{\partial x_{j}}\right) \\
\sigma_{\epsilon}=1.2, \quad C_{2}=1.9
\end{gathered}
$$

And,

$$
C_{\mu}=\frac{1}{A_{0}+A_{s} \frac{k U^{\prime}}{\epsilon}}
$$

Where,

$$
\begin{gathered}
U^{\prime}=\sqrt{S_{i j} S_{i j}+\widetilde{\Omega}_{i j} \widetilde{\Omega}_{i j}} \\
\left\{\begin{array}{c}
\widetilde{\Omega}_{i j}=\Omega_{i j}-2 \epsilon_{i j k} \omega_{k} \\
\Omega_{i j}=\bar{\Omega}_{i j}-\epsilon_{i j k} \omega_{k}
\end{array}\right.
\end{gathered}
$$

The constants in Eq. (19) are as follows:

$$
A_{0}=4.04, \quad A_{S}=\sqrt{6} \cos \phi
$$


Where,

$$
\phi=\frac{1}{3} \cos ^{-1}(\sqrt{6} W), \quad W=\frac{S_{i j} S_{j k} S_{k i}}{\sqrt{S_{i j} S_{i j}}}
$$

Finally, the default constants are as follows:

$$
\begin{aligned}
& C_{1 \epsilon}=1.44, \quad \sigma_{k}=1.0, \\
& \sigma_{\epsilon}=1.2, \quad C_{2}=1.9
\end{aligned}
$$

As shown in Figure 18, the simulations done for the perfect case at a blowing ratio of 0.45 match the experimental data well. It can be seen that the centerline effectiveness in the vicinity of the hole exit $(\mathrm{x} / \mathrm{D}=0.5)$ is under predicted by about $22 \%$. This might be due to the turbulence model predicting high mixing at the hole exit. The lateral effectiveness shows a maximum deviation of $20 \%(\mathrm{x} / \mathrm{D}=1.5)$ from the experimental data with excellent agreement overall. The simulations for the perfect case were all performed using the realizable k-epsilon model with the enhanced wall treatment.

Solutions acquired from the simulations of the imperfect case agrees well with experimental data, Figure 19 [33]. As can be seen, the lateral effectiveness is over predicted in the vicinity of the hole exit (from $\mathrm{x} / \mathrm{D}=0$ to $\mathrm{x} / \mathrm{D}=0.5$ ). The simulations for the imperfect case were performed using the SST k-omega for blowing ratios of 0.45 and 0.9 and the realizable k-epsilon turbulence model with the enhanced wall treatment was used for a blowing ratio of 1.25. Different turbulence models were used for the imperfect case because they provided better agreement with the experimental data at different blowing ratios. 


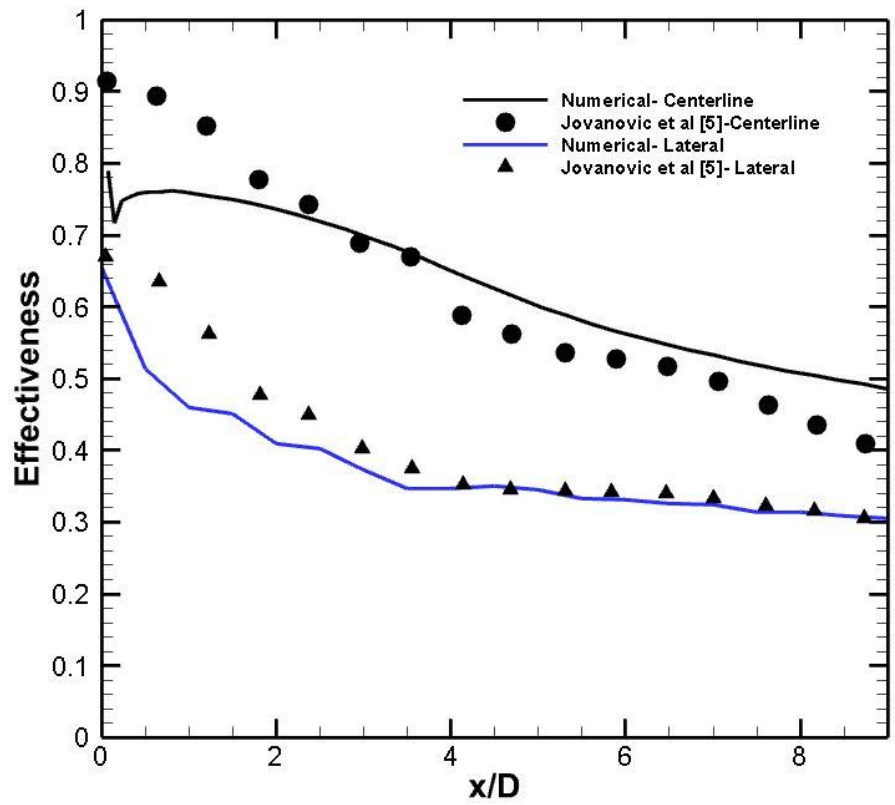

Figure 18. Perfect Case Validation $(\mathrm{M}=0.45, \mathrm{DR}=1)$

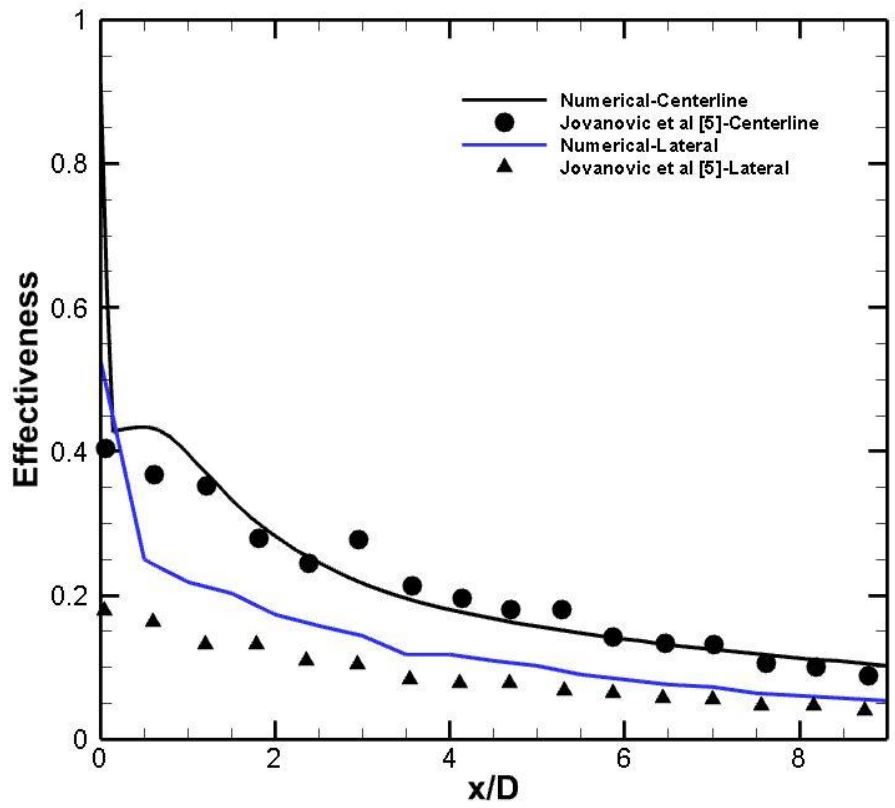

Figure 19. Imperfect Case Validation $(\mathrm{M}=0.9, \mathrm{DR}=1)$ 


\section{Effect of Imperfection Position}

In this section, the effect of hole of imperfection position on the film cooling effectiveness will be investigated. In order to monitor the effects of the imperfection, a perfect case is used as a baseline with a hole inclination angle of $35^{\circ}$. The hole imperfection locations investigated are 1, 2, 3 and 4 diameters from the hole exit leading edge at the blowing ratios of $0.45,0.90$ and 1.25, respectively. The effect of the imperfection position will be investigated with the use of laterally averaged and centerline film cooling effectiveness plots coupled with flow structure contours and velocity fields.

\section{Laterally averaged film cooling effectiveness}

The predicted laterally averaged film cooling effectiveness results taken along the lateral ( $\mathrm{Z}$ axis) from $0.5 \mathrm{D}$ to $0.5 \mathrm{D}$ from the centerline are displayed in Figure 20 for the perfect and imperfect cases at the blowing ratios of $\mathrm{M}=0.45,0.90$ and 1.25 for imperfection locations of $1,2,3$ and $4 \mathrm{D}$, respectively.

Results for a blowing ratio of $M=0.45$ are presented in Figure 20 (a). It is shown that the location of the imperfection inside the hole does have an influence on film cooling effectiveness. It can be seen that as the imperfection is located farther away from the hole exit, the laterally averaged effectiveness is improved. When the imperfection is located 1D away from the hole exit, it can be observed that the effectiveness almost at all locations downstream is deteriorated compared to the perfect case. However, an improvement in effectiveness is achieved when the imperfection is placed farther inside the hole. In fact, when the imperfection is located 4D from the hole exit, a $46.5 \%$ improvement in laterally averaged effectiveness at $\mathrm{x} / \mathrm{D}=1$ is achieved. It can be seen that for imperfection positions of 2,3 and 4 , the laterally averaged effectiveness is better near the hole than the perfect case until about a downstream distance of $\mathrm{x} / \mathrm{D}=4,8$ and 9 respectively.

Figure 20 (b) displays the results for a blowing ratio of $M=0.90$. It can be seen that the same trends that occurred for a blowing ratio of $\mathrm{M}=0.45$ reappear. However, the laterally averaged effectiveness outperforms the perfect case for shorter downstream distance compared to $\mathrm{M}=0.45$ until the deterioration occurs. The imperfection located 2D from the hole exit shows a $123.8 \%$ improvement at $\mathrm{x} / \mathrm{D}=0.5$. However, it can be seen that for imperfection positions of 1,2, 3 and 4, the laterally averaged effectiveness is better near the hole than the perfect case until about a downstream distance of $\mathrm{x} / \mathrm{D}=1.4,3.95,5.75$ and 5.75, respectively. Figure 20 (c) displays the results for a blowing ratio of $M=1.25$. It can be seen that the laterally averaged effectiveness plots start to converge as one curve. However, the same trends observed at M=0.45 and 0.9 seem to persist at higher blowing ratios. The imperfection located $2 \mathrm{D}$ and $3 \mathrm{D}$ from the hole exit shows a maximum $33 \%$ improvement at $\mathrm{x} / \mathrm{D}=1.5$. However, it can be seen that for imperfection positions of 1,2 
and 3, the laterally averaged effectiveness is better near the hole than the perfect case until about a downstream distance of $\mathrm{x} / \mathrm{D}=2.38,3.94$ and 3.94 respectively.

\section{Centerline film cooling effectiveness}

The predicted centerline effectiveness results taken from an $\mathrm{x} / \mathrm{D}=0$ to $\mathrm{x} / \mathrm{D}=40$ along the centerline are displayed in Figure 21 for the perfect and imperfect cases at the blowing ratios of $\mathrm{M}=0.45,0.90$ and 1.25 for imperfection locations of $1,2,3$ and $4 \mathrm{D}$, respectively.

Results for a blowing ratio of $M=0.45$ are presented in Figure 21 (a). The centerline effectiveness plots indicate that an improvement in effectiveness is achieved only for imperfections located 3D and 4D from the hole exit until about a downstream distance of about $\mathrm{x} / \mathrm{D}=10$. The imperfection located 4D from the hole exit shows a maximum $23.4 \%$ improvement at $x / D=5.2$. It can be seen that for imperfection positions of 3 and 4 , the centerline effectiveness is better near the hole than the perfect case until about a downstream distance of $\mathrm{x} / \mathrm{D}=8.3$ and 17.4 respectively. However, it can be observed that for the $4 \mathrm{D}$ case the centerline effectiveness starts improving again after $\mathrm{x} / \mathrm{D}=17.4$.

Results for a blowing ratio of $M=0.90$ are presented in Figure 21 (b). At this blowing ratio, it can be seen that the presence of an imperfection has detrimental effects on the centerline effectiveness. Plots indicate that imperfections located 3D and 4D from the hole exit are able to match the centerline effectiveness of the perfect case until about a downstream distance of about $\mathrm{x} / \mathrm{D}=7.9$ until deterioration happens. The imperfection located $4 \mathrm{D}$ from the hole exit shows a maximum $70.5 \%$ deterioration at $\mathrm{x} / \mathrm{D}=11.2$. The only location where the imperfection was seen to outperform the perfect case was in the vicinity of the hole exit. Results for a blowing ratio of $M=1.25$ are presented in Figure 21 (c). The centerline effectiveness plots indicate that an improvement in effectiveness is achieved only for imperfections for all locations from the hole exit until about a downstream distance of about $x / D=5$. The imperfection located $1 \mathrm{D}$ and $2 \mathrm{D}$ from the hole exit shows a maximum $43.5 \%$ improvement at $\mathrm{x} / \mathrm{D}=1.3$.

\section{Flow structure}

Figure 22 shows the temperature contours and the velocity fields for $\mathrm{M}=0.45$ at hole inclination angles of $35^{\circ}$ at the downstream locations $x / D=1$ for the perfect case and the imperfection location of $1 \mathrm{D}, 2 \mathrm{D}, 3 \mathrm{D}$ and $4 \mathrm{D}$. The temperature contours extend $1.5 \mathrm{D}$ horizontally in the $\mathrm{z}$ direction to both sides from the centerline and 3D vertically in the y direction. It can be seen from Figure 22 (b) that the CRVP for the imperfect case located 1D from the hole exit is much more intense than that of the jet of the perfect case or other imperfection cases. Furthermore, it can be seen that as the imperfection is position further inside the hole, the coolant is closer to the surface and spreads more laterally the perfect case thus concentrating the 
coolest portion of the jet near the surface. For instance, it can be observed that for an imperfection located $4 \mathrm{D}$ inside the hole, the coolant reaches a height of $\mathrm{y} / \mathrm{D}=0.77$ compared to $\mathrm{y} / \mathrm{D}=0.85$ for the perfect case and spreads from $\mathrm{z} / \mathrm{D}=-0.89$ to $\mathrm{z} / \mathrm{D}=0.85$ compared to $\mathrm{z} / \mathrm{D}=-0.7$ to $\mathrm{z} / \mathrm{D}=0.67$.

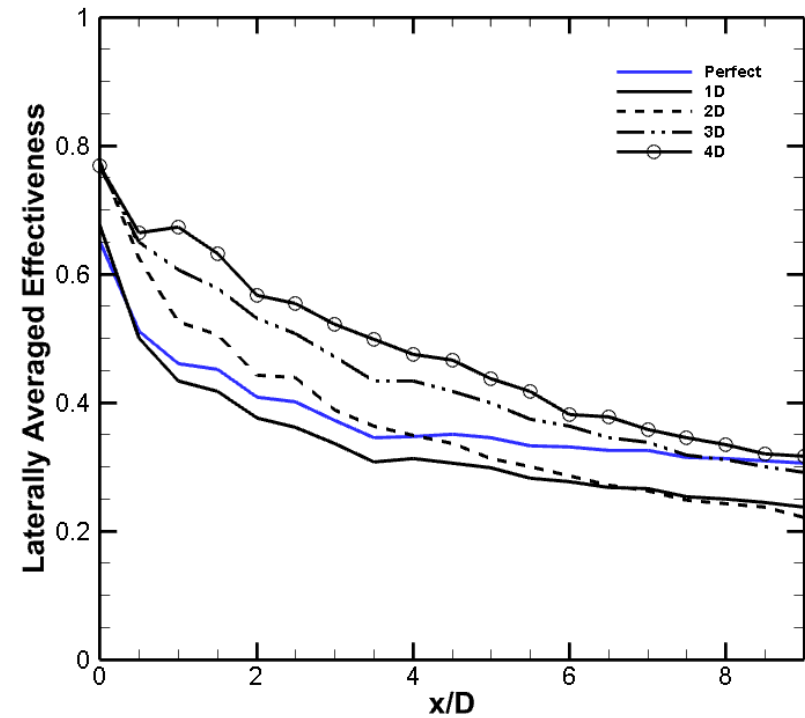

a) $\mathrm{M}=0.45, \mathrm{DR}=1$

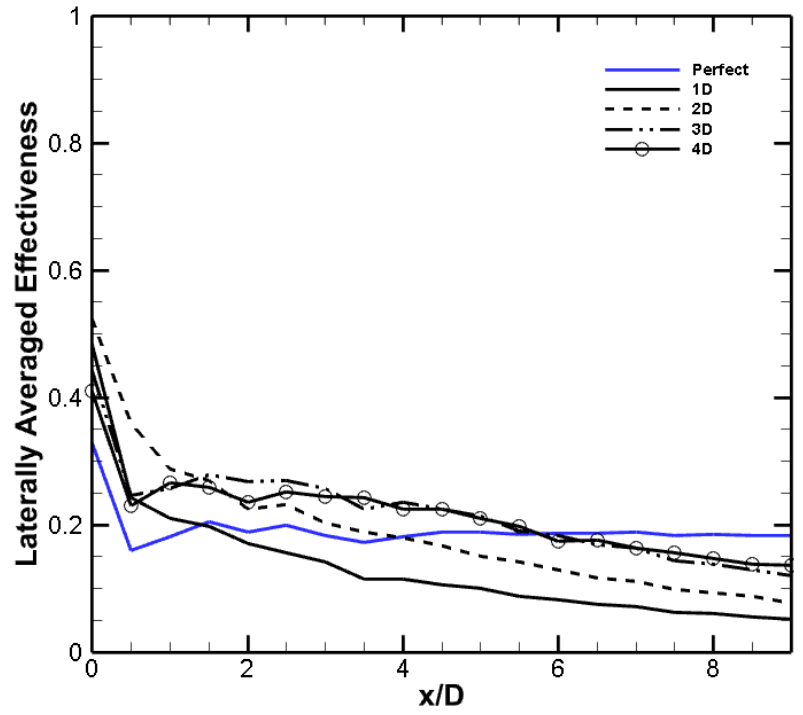

b) $\mathrm{M}=0.9, \mathrm{DR}=1$

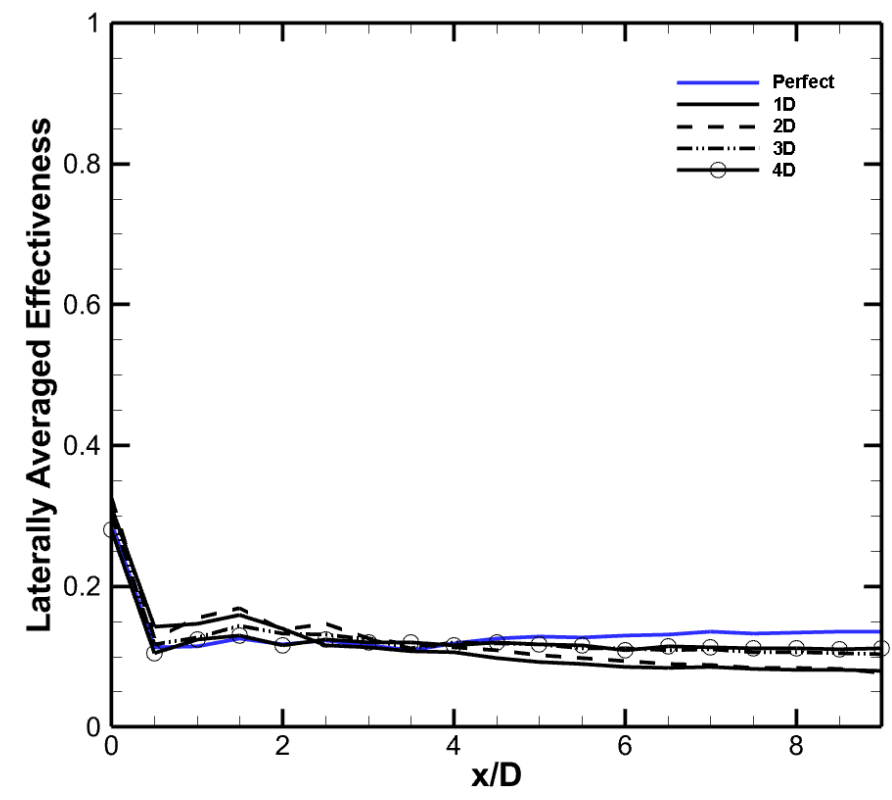

c) $\mathrm{M}=1.25, \mathrm{DR}=1$

Figure 20. Laterally Averaged Effectiveness- Effect of Imperfection Position 


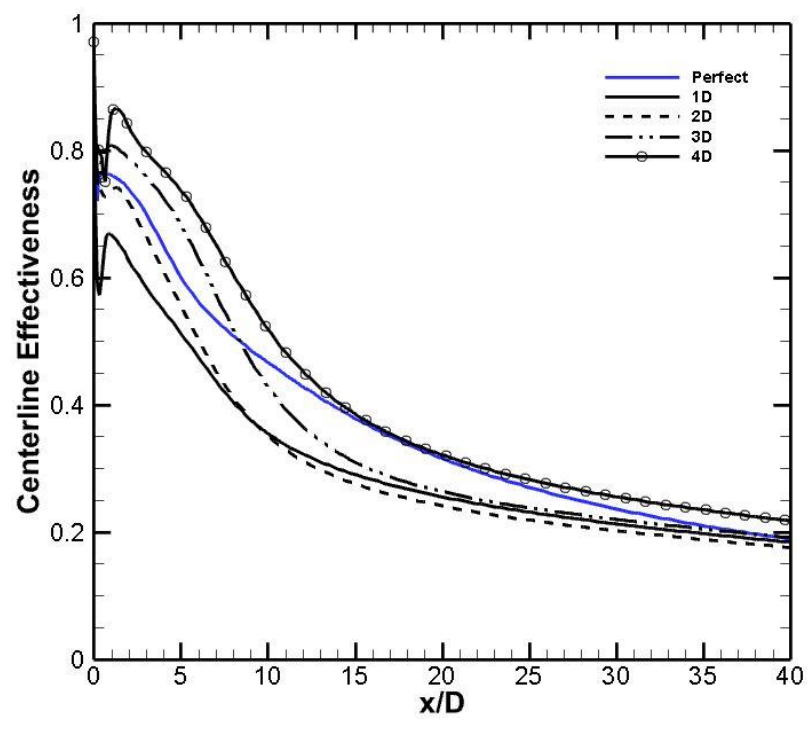

a) $\mathrm{M}=0.45, \mathrm{DR}=1$

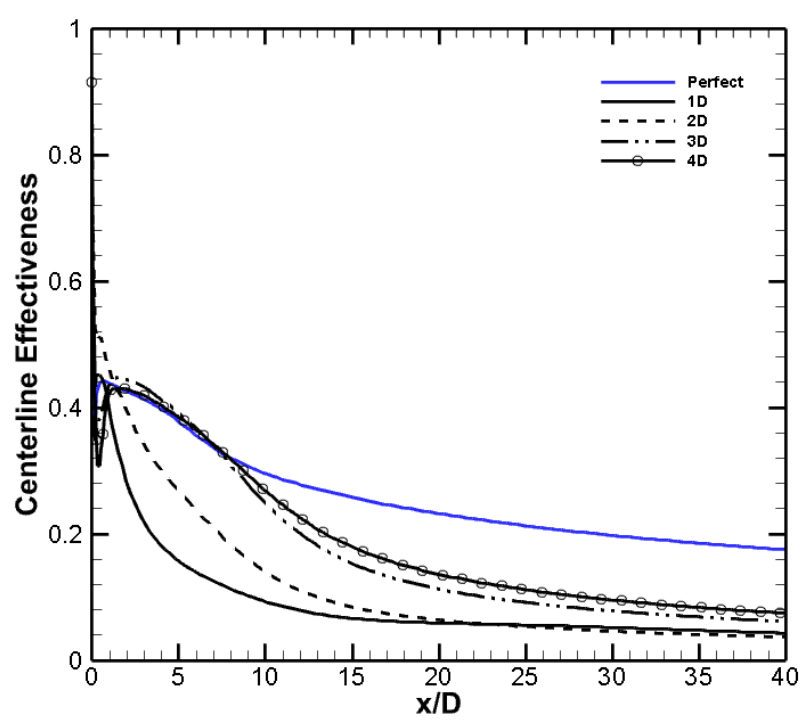

b) $\mathrm{M}=0.9, \mathrm{DR}=1$

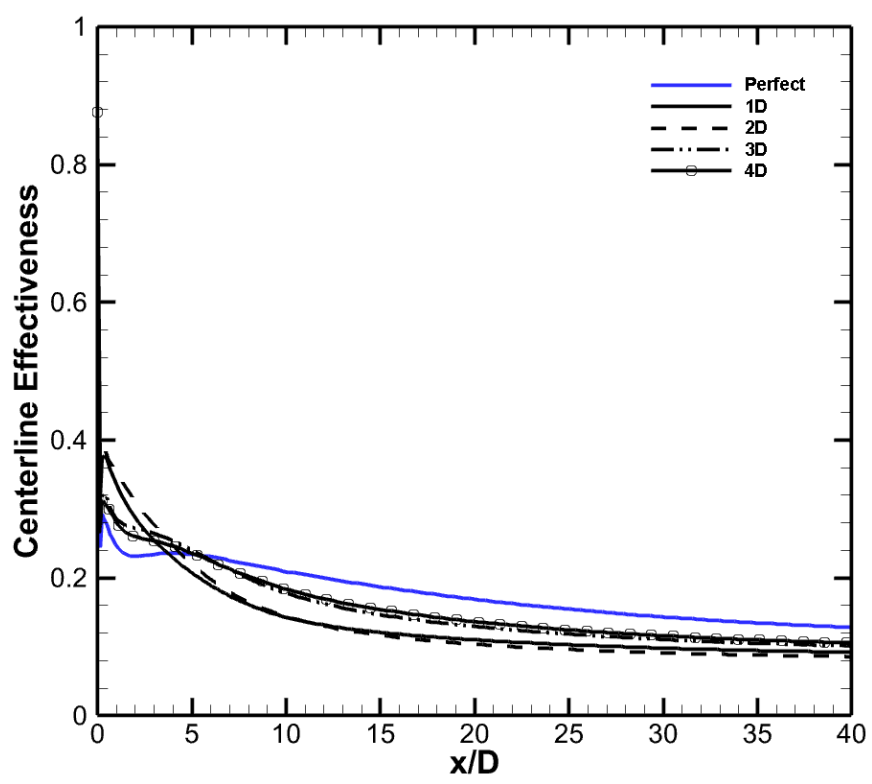

c) $\mathrm{M}=1.25, \mathrm{DR}=1$

Figure 21. Centerline Effectiveness- Effect of Imperfection Position 


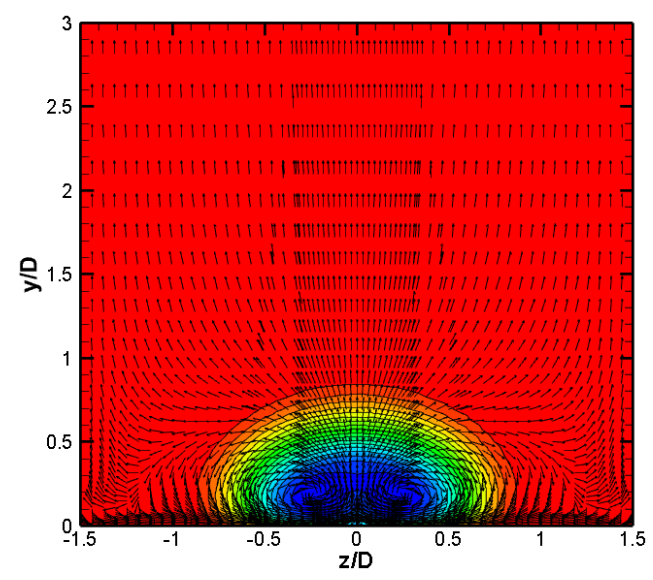

a) Perfect

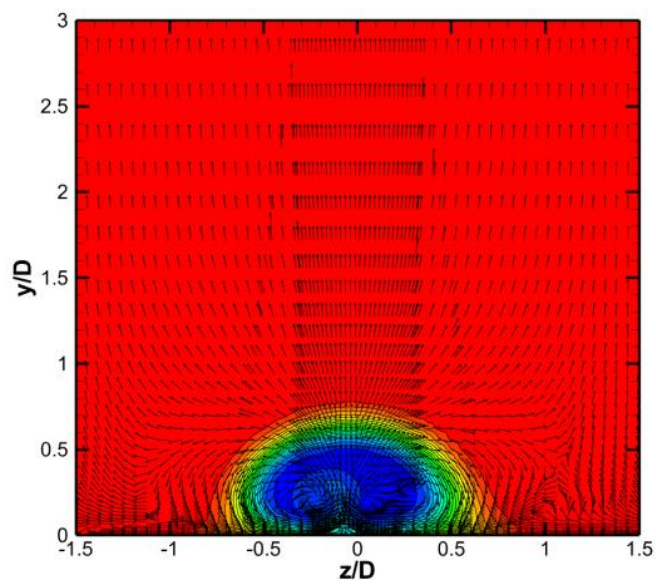

c) $2 \mathrm{D}$

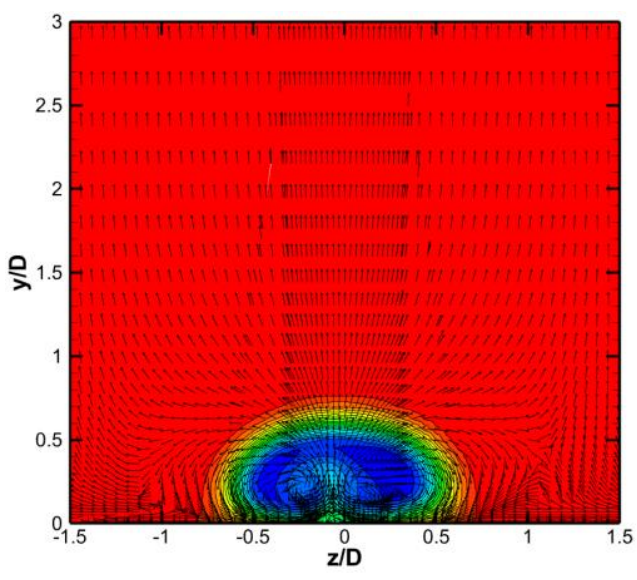

b) $1 \mathrm{D}$

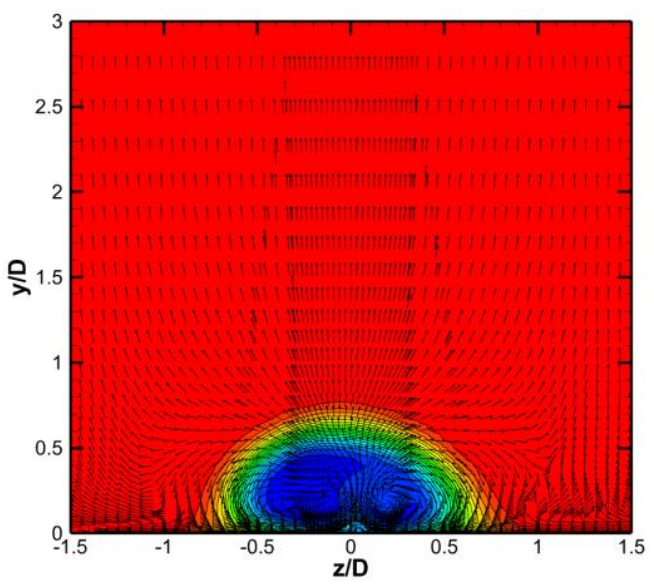

d) $3 \mathrm{D}$

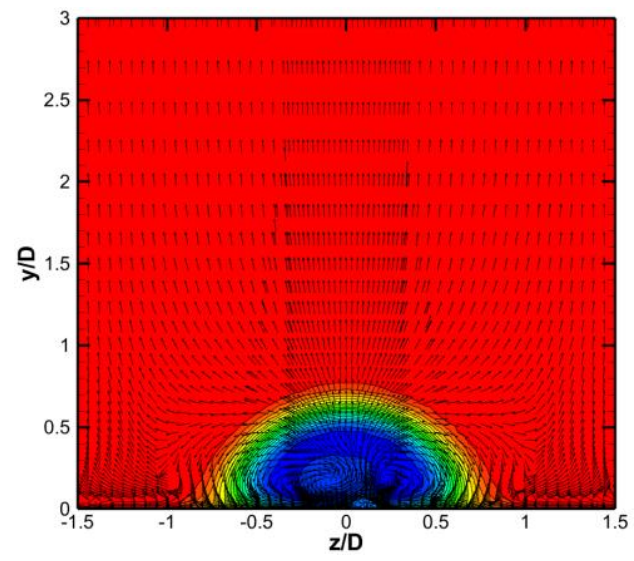

e) $4 \mathrm{D}$

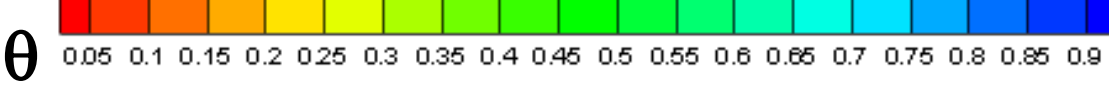

Figure 22. Dimensionless Temperature Contours and Velocity Vectors at $\mathrm{x} / \mathrm{D}=1$ for $\mathrm{M}=0.45$ for Perfect and Imperfect Location Cases 


\section{Effect of Hole Inclination}

In this section, the effect of hole inclination angle with the imperfection on the film cooling effectiveness will be investigated. In order to monitor the effects of the imperfection, a perfect case is used as a baseline. The hole inclination angles investigated are $35^{\circ}, 45^{\circ}$ and $55^{\circ}$ at the blowing ratios of $0.45,0.90$ and 1.25 , respectively. The effect of hole inclination angle with the imperfection will be investigated with the use of laterally averaged and centerline film cooling effectiveness plots coupled with flow structure contours and velocity fields.

\section{Laterally averaged film cooling effectiveness}

The predicted laterally averaged film cooling effectiveness results taken along the lateral ( $\mathrm{Z}$ axis) from $0.5 \mathrm{D}$ to $0.5 \mathrm{D}$ from the centerline are displayed in Figure 23 for the perfect and imperfect cases at the blowing ratios of $\mathrm{M}=0.45,0.90$ and 1.25 for hole inclinations of $35^{\circ}, 45^{\circ}$ and $55^{\circ}$, respectively.

Results for a blowing ratio of $M=0.45$ are presented in Figures 23 (a), (b) and (c). It is shown that the imperfection causes a deterioration in the laterally averaged film cooling effectiveness for all inclination angles compared to the perfect case. The decrease in lateral effectiveness is shown not to be severe for a hole inclination angle of $35^{\circ}$ with a maximum deterioration of $26 \%$. The maximum laterally averaged effectiveness deterioration for $45^{\circ}$ and $55^{\circ}$ are $43 \%$ and $53 \%$, respectively.

Figures 23 (d), (e) and (f) display the results for a blowing ratio of $M=0.90$. It can be seen that for a hole inclination of $35^{\circ}$, the imperfect case outperforms the perfect case in the vicinity of the hole from $x / D=0$ to $x / D=1.5$. As the hole inclination angle is increased, the region where the imperfection case outperforms the perfect case decreases slightly. The maximum laterally averaged effectiveness deterioration for all hole inclinations is around $87.5 \%$ at $\mathrm{x} / \mathrm{D}=9$. Results for a blowing ratio of $\mathrm{M}=1.25$ are presented in Figures 23 (g), (h) and (i). The same outperformance shown at the hole vicinity by the imperfect case at $\mathrm{M}=0.90$ is observed here as well. However, this is seen to be the case for a longer distance downstream of the hole exit. For a hole inclination angle of $35^{\circ}$, the imperfect case outperforms the perfect case from $\mathrm{x} / \mathrm{D}=0$ to $\mathrm{x} / \mathrm{D}=3.4$. Furthermore, for a hole inclination angle of $35^{\circ}$, the imperfect case outperforms the perfect case from $x / D=0$ to $x / D=2.3$. The maximum laterally averaged effectiveness deterioration for $35^{\circ}, 45^{\circ}$ and $55^{\circ}$ are $50 \%, 79 \%$ and $75 \%$, respectively.

\section{Centerline film cooling effectiveness}

The predicted centerline film cooling effectiveness results are presented in Figure 24 for the perfect and imperfect cases at the blowing ratios of $\mathrm{M}=0.45,0.90$ and 1.25 for hole inclinations of $35^{\circ}, 45^{\circ}$ and $55^{\circ}$, 
respectively. Results for a blowing ratio of $\mathrm{M}=0.45$, Figures 24 (a), (b) and (c) reveal a deterioration in effectiveness when the imperfection is present. It can be seen that as the hole inclination angle is increased, the centerline effectiveness for the perfect case varies slightly. However, the centerline effectiveness for the imperfect cases shows a dependence on the hole inclination angle. For these cases, the most severe deterioration occurs immediately at the hole exit $\mathrm{x} / \mathrm{D}=0$ and gradually approaches the perfect case as it reaches $\mathrm{x} / \mathrm{D}=40$. A maximum centerline effectiveness deterioration of $25 \%, 34 \%$ and $45 \%$ at a hole inclination angle of $35^{\circ}, 45^{\circ}$ and $55^{\circ}$, respectively is seen.

Results for a blowing ratio of $M=0.90$ shown Figures 24 (d), (e) and (f) reveal a more pronounced decrease in effectiveness for the imperfect case when compared to the baseline case. Minute changes are observed for the perfect case when the hole inclination angle is changed. The most profound changes for the imperfect case occurs in the vicinity of the hole exit between $x / D=0$ and 10 where a noticeable decrease in centerline effectiveness is observed. A maximum centerline effectiveness deterioration of $56 \%(\mathrm{x} / \mathrm{D}=5), 76 \%(\mathrm{x} / \mathrm{D}=5)$ and $89 \%(x / D=1)$ at a hole inclination angle of $35^{\circ}, 45^{\circ}$ and $55^{\circ}$, respectively is seen.

Figures $24(\mathrm{~g})$, (h) and (i) present centerline effectiveness plots for a blowing ratio of $M=1.25$. It is shown that the imperfect case has higher centerline effectiveness at the hole exit vicinity compared to the perfect case. As the hole inclination angle is increased the region where the performance of the imperfection case outperforms the baseline case reduces from $\mathrm{x} / \mathrm{D}=5$ to 1 . Similarly to the previous cases, the hole imperfection shows greater dependence on the hole inclination angle than the baseline case. It can be seen that as the hole angle is increased, the film effectiveness deterioration is more profound. A maximum centerline effectiveness deterioration of $38 \%, 63 \%$ and $74 \%$ at a hole inclination angle of $35^{\circ}, 45^{\circ}$ and $55^{\circ}$, respectively is observed at $\mathrm{x} / \mathrm{D}=10$.

The hole imperfection reduces the cross sectional area of the hole causing the coolant jet velocity to increase. This causes an unwanted momentum increase in the y direction enhancing jet lift off. As the hole inclination angle is increased, the vertical component of the jet momentum becomes considerably larger than the horizontal component. Based on the discussion above, the imperfect case at a blowing ratio of 0.90 and at a hole inclination angle of $55^{\circ}$ showed the greatest centerline effectiveness deterioration compared to the perfect case. This case will be studied in more depth in the next section due to this reason.

\section{Flow structure}

In order to better comprehend the results provided in Figure 24, the centerline effectiveness plots need to be compared with temperature contours in order to visualize the flow structure. Figures 25 and 26 show the temperature contours and the velocity fields for $M=0.90$ at hole inclination angles of 35,45 and 55 at the downstream locations $x / D=1$ and 5 , respectively. The temperature contours extend $1.5 \mathrm{D}$ horizontally in the 
$\mathrm{z}$ direction to both sides from the centerline and 3D vertically in the y direction. It can be seen from Figure 25 that a location of $x / D=1$ the coolant jet for the imperfect case is much more compact than that of the jet of the perfect case. This is an indication that the jet did not recover from the acceleration caused by the hole imperfection. As the jet present in the imperfection case is more compact, the counter rotating vortex pair (CRVP) is seen to be closer to each other. This causes the jet to be subject to a stronger lift when the CRVP assist each other. This can be seen from the velocity fields in Figure 25 where the vectors pointing upwards cover a bigger portion of the jet for the imperfect case compared to the perfect case. Haven and Kurosaka [38] demonstrated that the inlet geometry influences how far apart the CRVP are from each other. They showed that for high aspect ratio geometries (larger hole cross-stream dimension for the same crosssectional hole area), the counter rotating vortices are farther apart reducing the lift off effect. The opposite is true for low aspect ratio geometries. Moreover, the effect of the hole inclination angle for the imperfection case is clearly demonstrated in Figures 25 (d), (e) and (f). The jet remains attached to the plate at a hole inclination angle of $35^{\circ}$ and begins to detach at an angle of $45^{\circ}$.

Finally, the jet is seen to be fully detached at an angle of $55^{\circ}$. Figures 25 (a), (b) and (c) shows the dimensionless temperature contours for the perfect case. It can be observed that the flow structures changes slightly for the perfect case when the hole inclination angle is varied. It can be seen in Figure 26 that the shape of the CRVP for the imperfect case at $\mathrm{x} / \mathrm{D}=5$ is more distinct. At a hole inclination of $35^{\circ}$, the jet is seen to almost completely detach. For the hole inclination angles of $45^{\circ}$ and $55^{\circ}$, the jets are seen to be fully detached. Unlike the jet seen in figure 26 (e), the jet at a hole inclination angle of $55^{\circ}$ in figure 26 (f) is not axisymmetric along the centerline. The left vortex from the counter rotating vortex pair becomes larger than the right vortex which tilts slightly the jet counter clockwise. These effects most likely originate from the lateral sides of the imperfection as it was witnessed by Pan et al [16] with a lateral blockage placed inside the hole. Similarly to the results at the location of $x / D=1$, the dimensionless temperature contours for the perfect case at a location of $\mathrm{x} / \mathrm{D}=5$ show slight changes when the hole inclination angle is varied. 

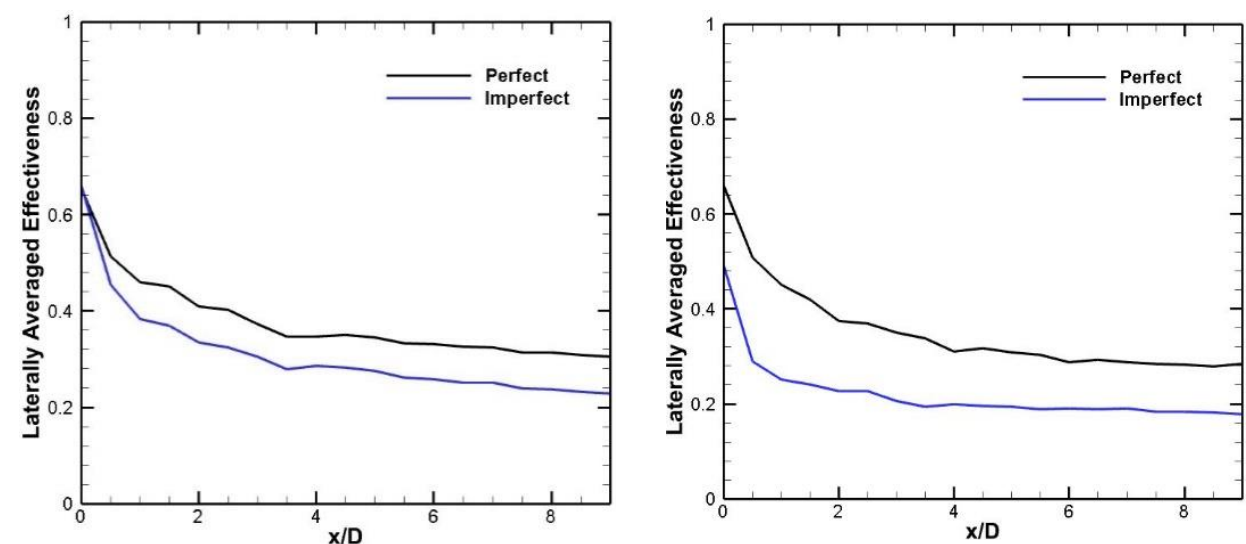

a) $\mathrm{M}=0.45,35^{\circ}$

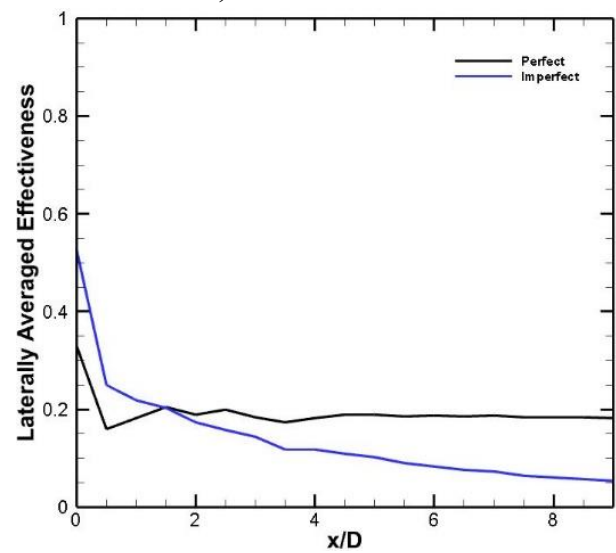

d) $\mathrm{M}=0.9,35^{\circ}$

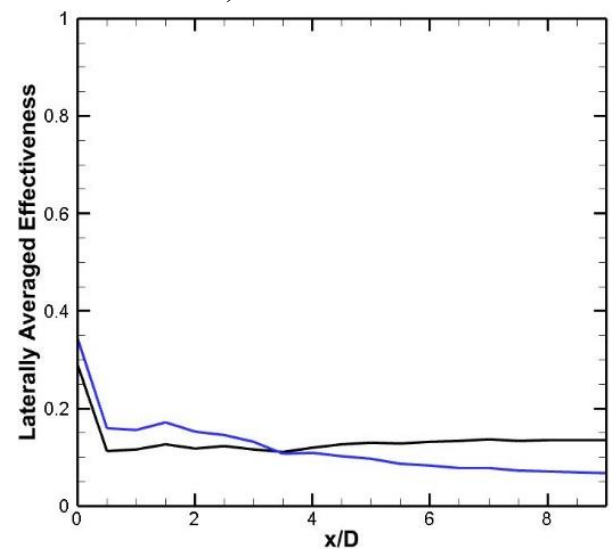

g) $\mathrm{M}=1.25,35^{\circ}$ b) $\mathrm{M}=0.45,45^{\circ}$

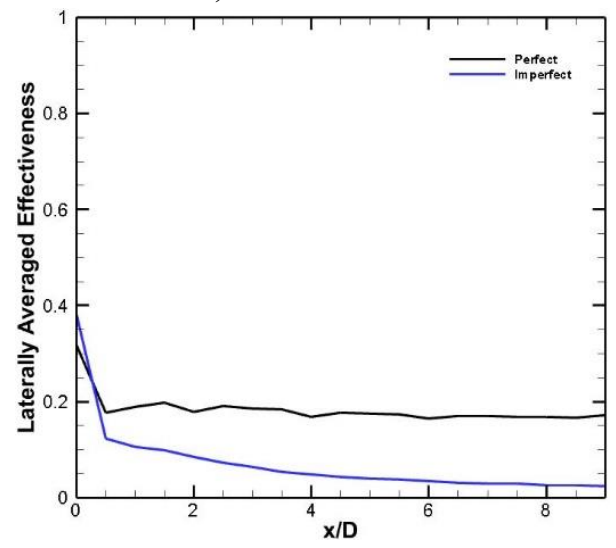

e) $\mathrm{M}=0.9,45^{\circ}$

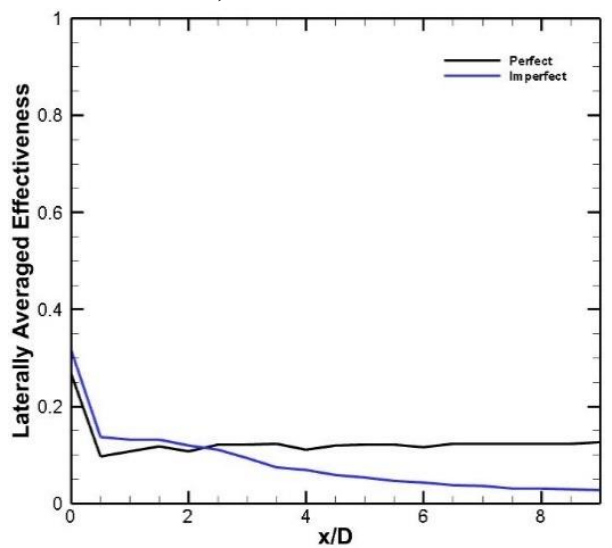

h) $\mathrm{M}=1.25,45^{\circ}$

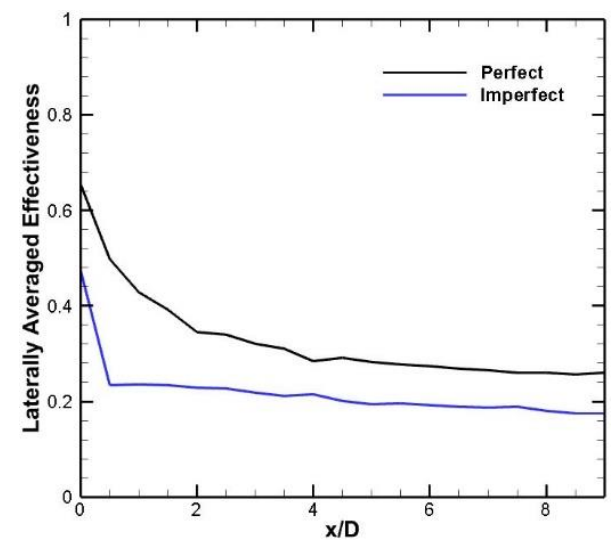

c) $\mathrm{M}=0.45,55^{\circ}$

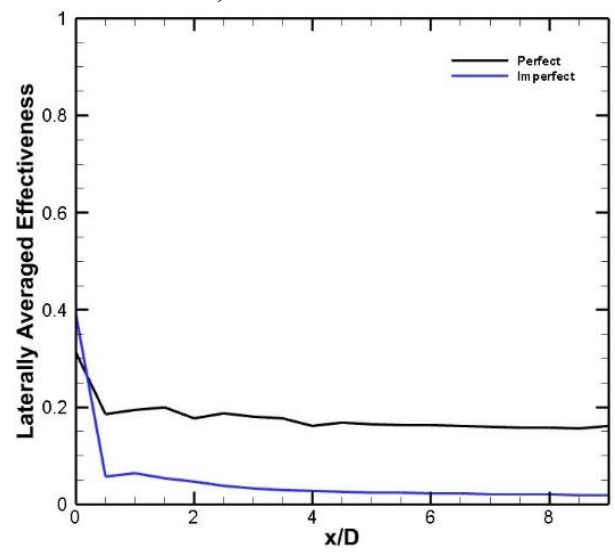

f) $\mathrm{M}=0.9,55^{\circ}$

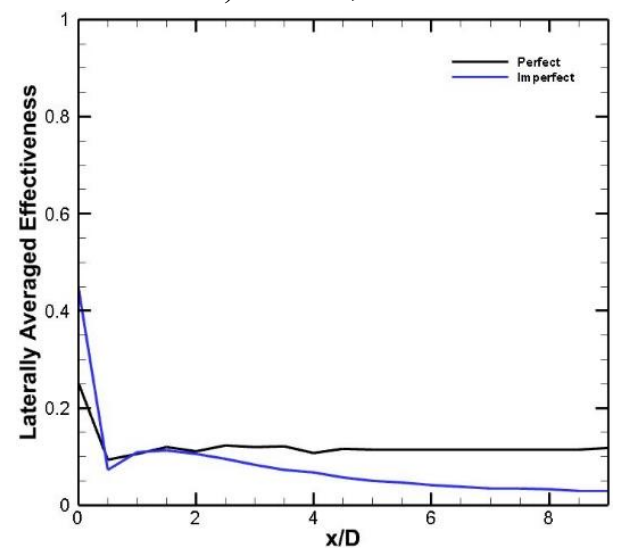

i) $\mathrm{M}=1.25,55^{\circ}$

Figure 23. Laterally Averaged Film Cooling Effectiveness; Perfect (Black) and Imperfect (Blue) Cases 

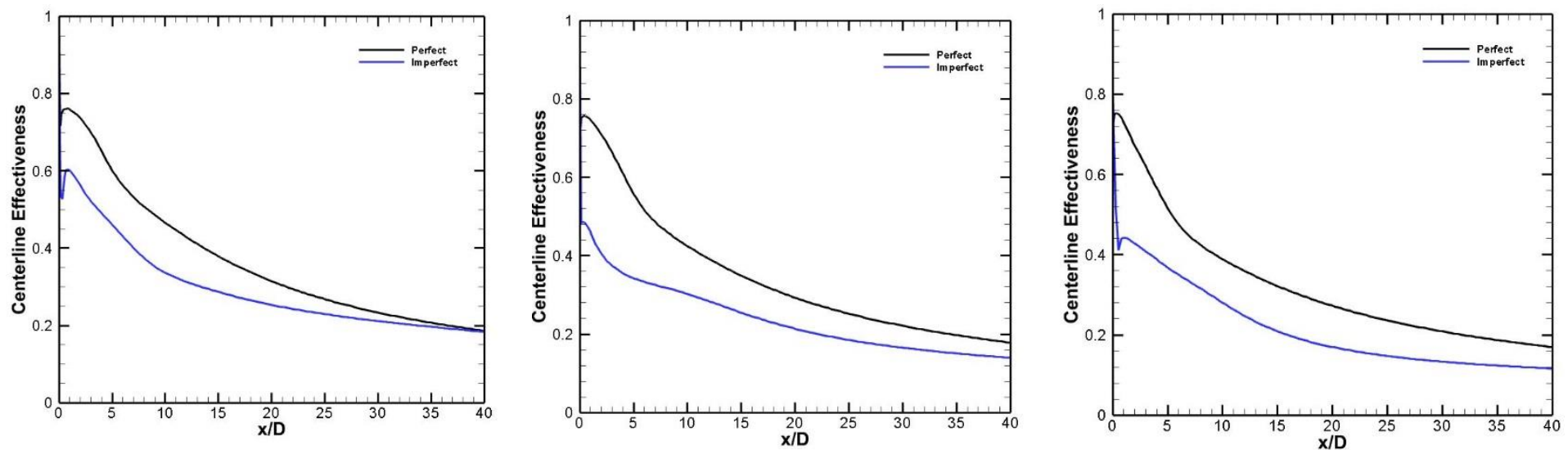

a) $\mathrm{M}=0.45,35^{\circ}$

b) $\mathrm{M}=0.45,45^{\circ}$
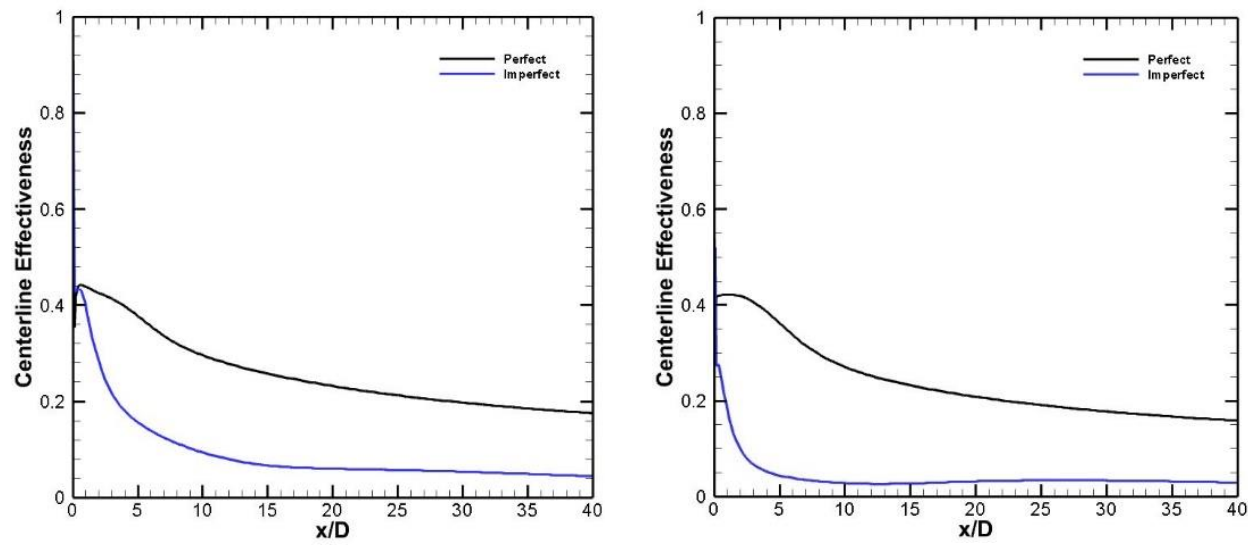

c) $\mathrm{M}=0.45,55^{\circ}$

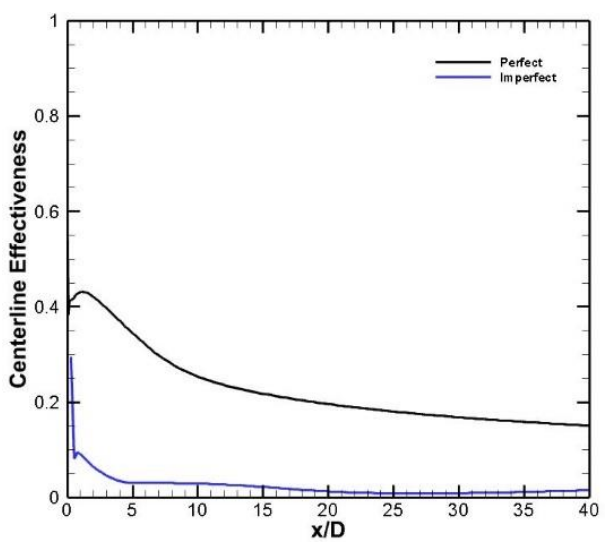

d) $\mathrm{M}=0.9,35^{\circ}$

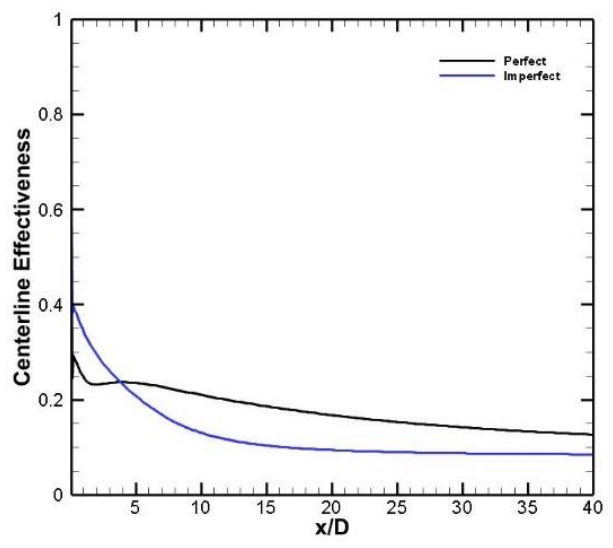

e) $\mathrm{M}=0.9,45^{\circ}$

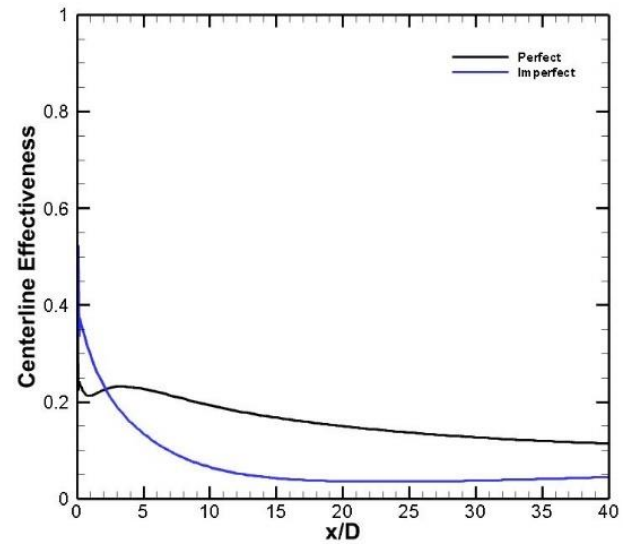

f) $\mathrm{M}=0.9,55^{\circ}$

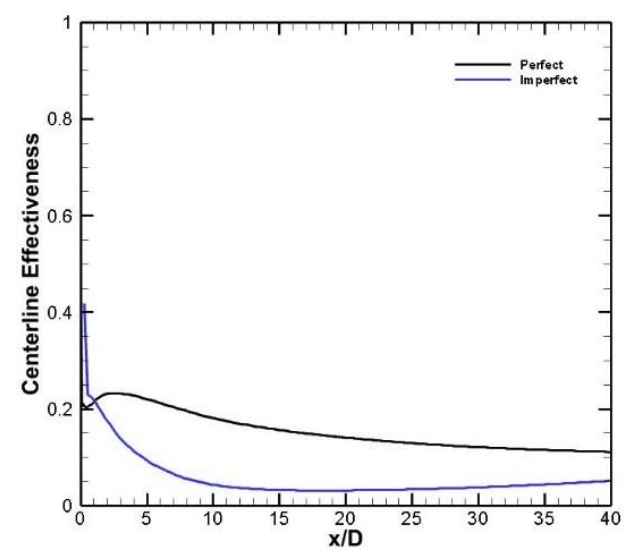

g) $\mathrm{M}=1.25,35^{\circ}$

h) $\mathrm{M}=1.25,45^{\circ}$

i) $\mathrm{M}=1.25,55^{\circ}$

Figure 24. Centerline Film Cooling Effectiveness; Perfect (Black) and Imperfect (Blue) Cases 


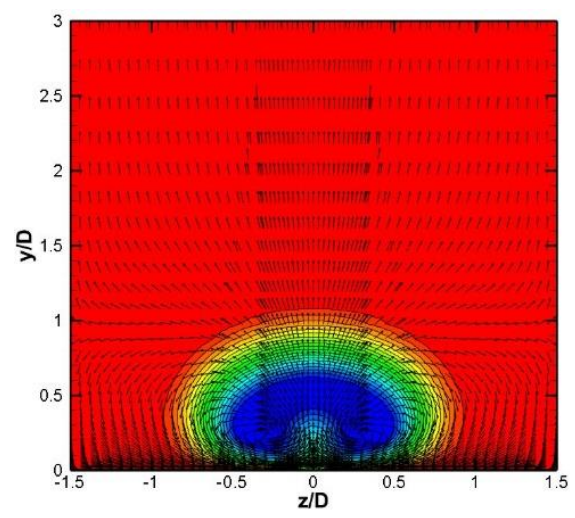

a) Perfect, $35^{\circ}$

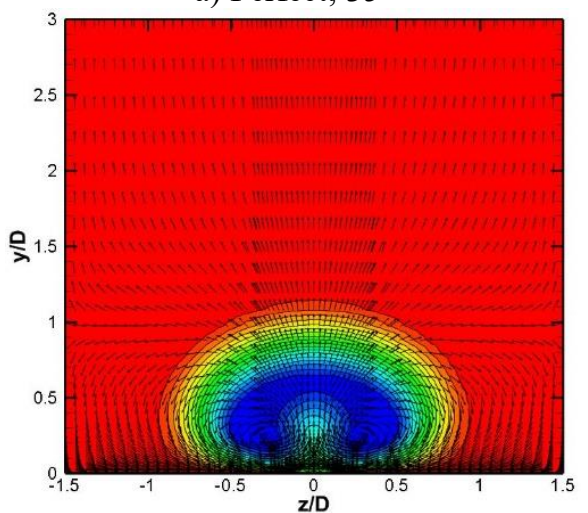

b) Perfect, $45^{\circ}$

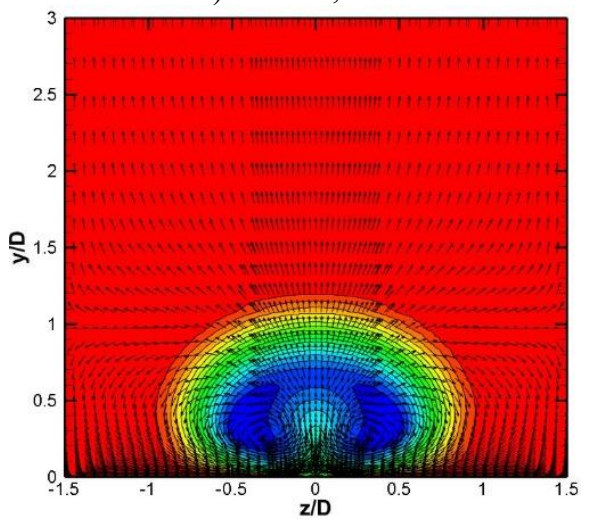

c) Perfect, $55^{\circ}$

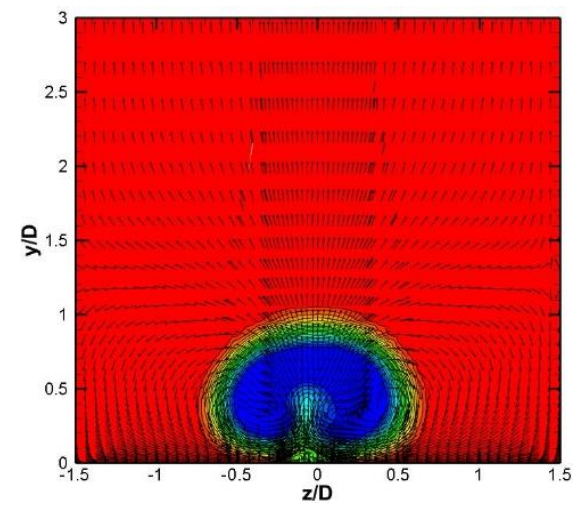

d) Imperfect, $35^{\circ}$

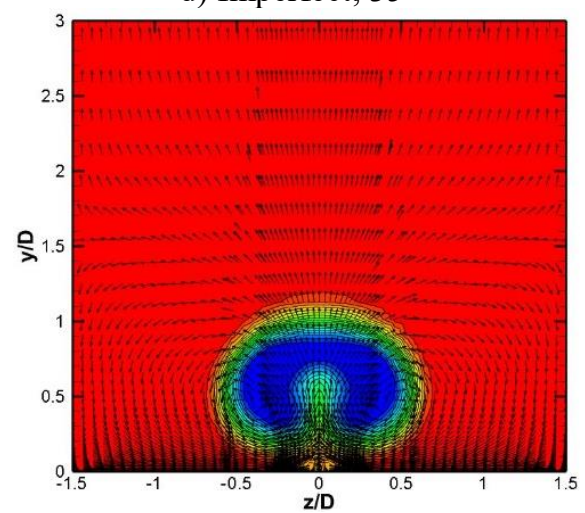

e) Imperfect, $45^{\circ}$

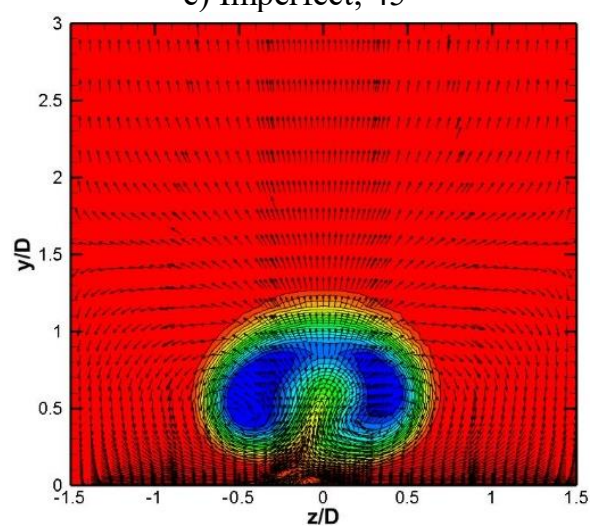

f) Imperfect, $55^{\circ}$

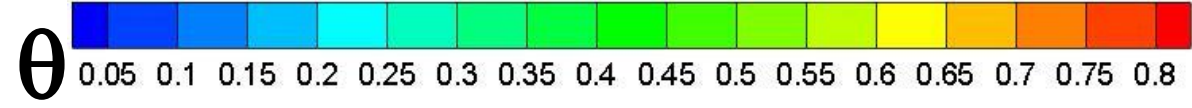

Figure 25. Dimensionless Temperature Contours and Velocity Vectors at $\mathrm{x} / \mathrm{D}=1$ for $\mathrm{M}=0.9$ for Perfect (left) and Imperfect (right) Cases 


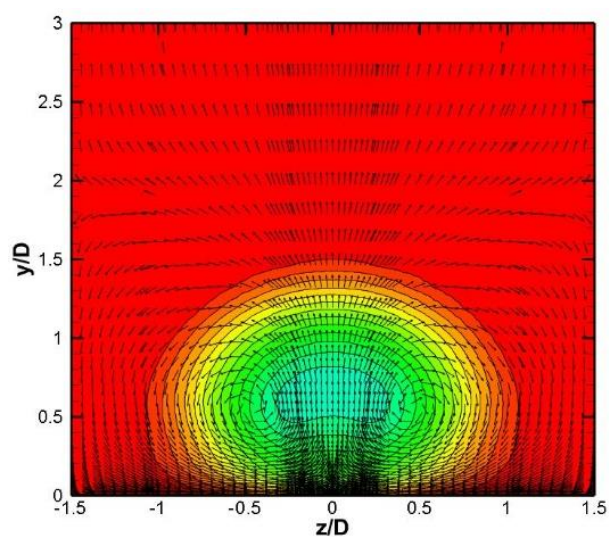

a) Perfect, $35^{\circ}$

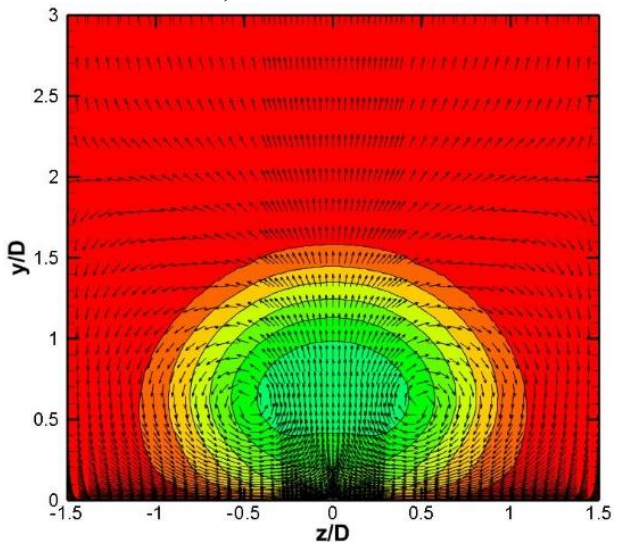

b) Perfect, $45^{\circ}$

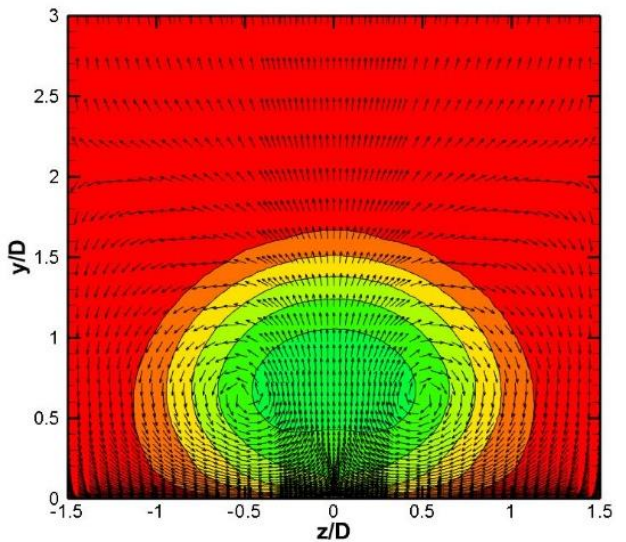

c) Perfect, $55^{\circ}$

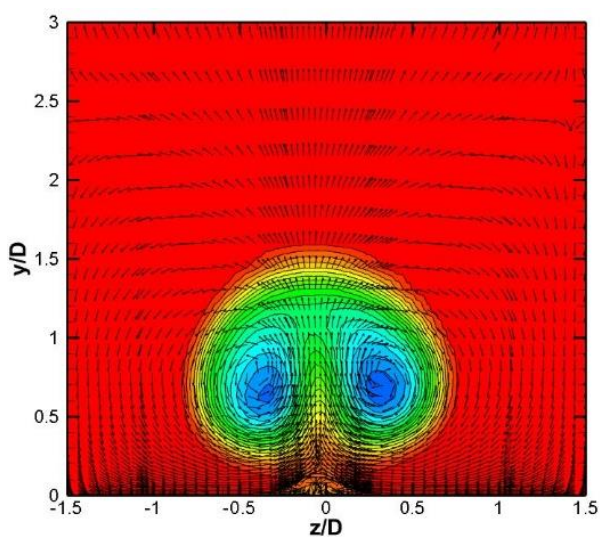

d) Imperfect, $35^{\circ}$

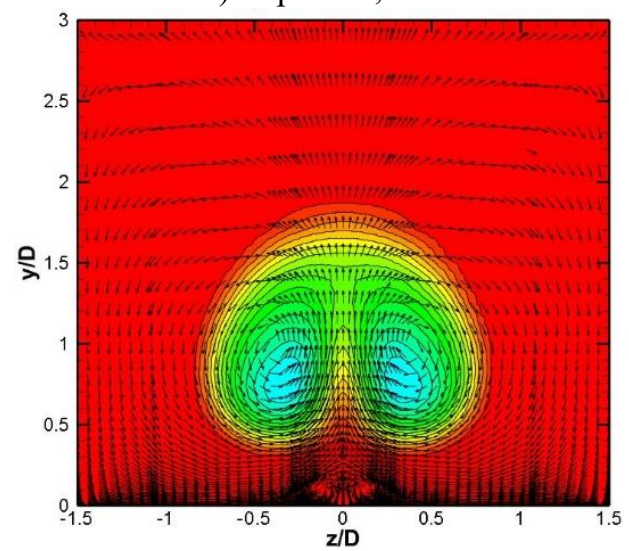

e) Imperfect, $45^{\circ}$

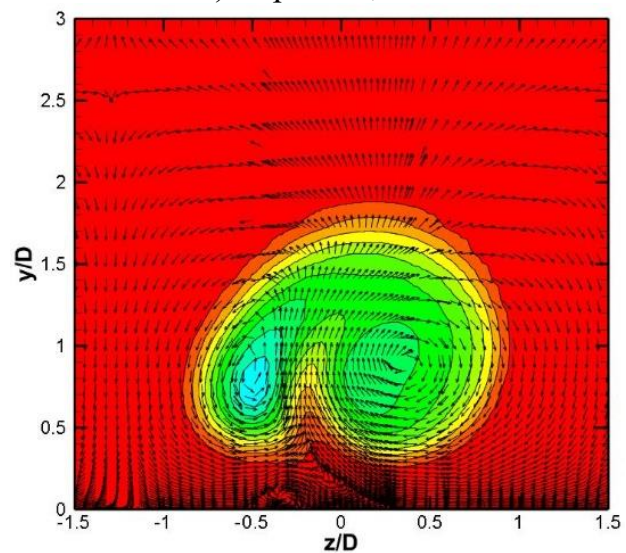

f) Imperfect, $55^{\circ}$

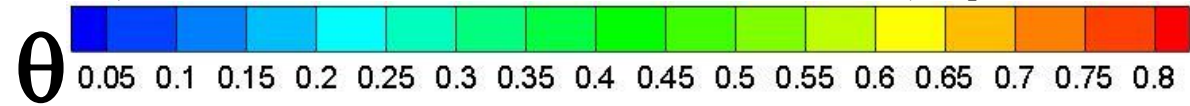

Figure 26. Dimensionless Temperature Contours and Velocity Vectors at $\mathrm{x} / \mathrm{D}=5$ for $\mathrm{M}=0.9$ for Perfect (left) and Imperfect (right) Cases 


\section{Effect of Imperfection Shape and Percent Obstruction}

In this section, the influence of a hole imperfection geometry on film cooling performance is numerically evaluated. Here, the hole imperfection due to laser percussion drilling is modelled as a half torus (circular) and with a rectangular profile. Three percent obstructions were investigated for both imperfection geometries: $26.3 \%, 35 \%$ and 40\%. Furthermore, every case was evaluated at three blowing ratios: 0.45 , 0.90 and 1.25. Each case is compared to a baseline case where the hole imperfection is absent. The effect of the imperfection geometry will be investigated with the use of laterally averaged and centerline film cooling effectiveness plots coupled with flow structure contours and velocity fields.

\section{Laterally averaged film cooling effectiveness}

The predicted laterally averaged film cooling effectiveness results taken along the lateral ( $\mathrm{Z}$ axis) from $0.5 \mathrm{D}$ to $0.5 \mathrm{D}$ from the centerline are displayed in Figure 27 for the perfect case and both imperfection geometries at the blowing ratios of $\mathrm{M}=0.45,0.90$ and 1.25 for the $26.3 \%, 35 \%$ and $40 \%$ obstructions.

Results for a blowing ratio of $M=0.45$ are presented in Figures 27 (a), (b) and (c). It is shown that the both imperfection geometry and imperfection percent obstruction have an influence on film cooling effectiveness. It can be seen that as the imperfection percent obstruction is increased, the deterioration of the laterally averaged effectiveness becomes more pronounced. It can be seen that for percent obstructions of $26.3 \%$ and $40 \%$, the circular and rectangular imperfections have similar effectiveness plots. However, for a percent obstruction of $35 \%$, the rectangular imperfection is observed to be more detrimental to the laterally averaged effectiveness than the circular imperfection. At $\mathrm{x} / \mathrm{D}=9$, a percent obstruction of $40 \%$ shows a maximum laterally averaged effectiveness deterioration of $70 \%$ for both imperfection profiles compared to the perfect case. Furthermore, at $\mathrm{x} / \mathrm{D}=4$, a percent obstruction of $35 \%$ shows a laterally averaged effectiveness deterioration of $17.6 \%$ and $43.9 \%$ for the circular and rectangular profiles compared to the perfect case, respectively.

Figures 27 (d), (e) and (f) display the results for a blowing ratio of $M=0.90$. It can be seen that the same trends for imperfection shape occurs where the laterally averaged effectiveness plots are similar and the circular shaped imperfection outperforms the rectangular shaped imperfection. However, for a percent obstruction of $40 \%$, it can be seen that an increase in effectiveness caused by the circular shaped imperfection where it outperforms the perfect case until $\mathrm{x} / \mathrm{D}=4.8$. For this percent obstruction, the circular shaped imperfection shows a maximum $131.1 \%$ improvement at $\mathrm{x} / \mathrm{D}=0.97$.

Figures 27 (g), (h) and (i) display the results for a blowing ratio of $M=1.25$. At this blowing ratio, it can be observed that neither percent obstruction nor imperfection shape have a significant influence on laterally averaged effectiveness. 


\section{Centerline film cooling effectiveness}

The predicted centerline effectiveness results taken from an $x / D=0$ to $x / D=40$ along the centerline are displayed in Figure 28 for the perfect and both imperfection geometries at the blowing ratios of $\mathrm{M}=0.45$, 0.90 and 1.25 for the $26.3 \%, 35 \%$ and $40 \%$ obstructions.

Results for a blowing ratio of $\mathrm{M}=0.45$ are presented in Figures 28 (a), (b) and (c). Similarly to the laterally averaged effectiveness plots, the centerline effectiveness plots indicate a significant deterioration in effectiveness occurs as the imperfection percent obstruction is increased. However, the shape of the imperfection does not have a significant effect on centerline effectiveness as was the case for the laterally averaged effectiveness. At $\mathrm{x} / \mathrm{D}=10$, it can be seen that a deterioration of $31.5 \%$ (circular shape only), $68.6 \%$ and $171.5 \%$ compared the perfect case at $26.3 \%, 35 \%$ and $40 \%$ obstructions, respectively.

Results for a blowing ratio of $M=0.90$ are presented in Figures 28 (d), (e) and (f). At this blowing ratio, it can be seen that the presence of an imperfection has the most detrimental effects on the centerline effectiveness. However, neither the shape nor the obstruction size have shown a significant influence with the exception $40 \%$ obstruction with a circular shape at the vicinity of the hole exit. At that obstruction size, the circular imperfection is seen to outperform the perfect case from $\mathrm{x} / \mathrm{D}=0.57$ to $\mathrm{x} / \mathrm{D}=2.1$. However, in general the trends are shown to be similar across obstruction sizes and shapes for this blowing ratio. At $\mathrm{x} / \mathrm{D}=10$, it can be seen that a deterioration of $262.5 \%, 533.2 \%$ and $735.7 \%$ compared the perfect case at $26.3 \%, 35 \%$ and $40 \%$ obstructions, respectively.

Results for a blowing ratio of $\mathrm{M}=1.25$ are presented in Figures $28(\mathrm{~g})$, (h) and (i). Similar to the previous blowing ratios, the centerline effectiveness plots indicate that a deterioration in effectiveness is more significant as the obstruction size is increased. All obstruction sizes show an outperformance in centerline effectiveness compared to the perfect case from $\mathrm{x} / \mathrm{D}=0$ to $\mathrm{x} / \mathrm{D}=4$ where a maximum of $48.6 \%$ improvement occurs at $\mathrm{x} / \mathrm{D}=0.3$.

Figures 29 and 30 present the centerline and laterally averaged effectiveness of the circular and rectangular shaped imperfection dealt separately; respectively. It can be seen that for low blowing ratios, the effects of the imperfection presence and size are more pronounced. Furthermore, at a blowing ratio of $\mathrm{M}=0.45$, the rectangular shaped imperfection has more adverse effects on effectiveness compared to the circular shaped imperfection. As the blowing ratio is increased, the differences caused by obstruction size diminishes for both shapes almost collapsing into a single curve. 


\section{Flow structure}

Figure 31 shows the temperature contours and the velocity fields for $\mathrm{M}=0.45$ at hole inclination angles of $35^{\circ}$ at the downstream locations $\mathrm{x} / \mathrm{D}=2$ for the circular and rectangular shaped imperfection with $26.3 \%$, $35 \%$ and $40 \%$ obstruction sizes. The temperature contours extend 1.5D horizontally in the $\mathrm{z}$ direction to both sides from the centerline and 3D vertically in the y direction. It can be seen from Figure 31 that the CRVP much more compact for high obstruction sizes thus enhancing jet lift off further. Moreover, it can be seen from Figures 31 (c) and (d) that even though the jet structures remain intact, core does not mix with the hot gases, the freestream reaches below the sides of the jets. Comparing the jets at an obstruction size of $35 \%$, it can be observed that the coolant for the circular shaped imperfection reaches a height of $y / D=$ 0.85 compared to $\mathrm{y} / \mathrm{D}=0.86$ for the rectangular shaped imperfection and spreads from $\mathrm{z} / \mathrm{D}=-0.65$ to $\mathrm{z} / \mathrm{D}=0.48$ compared to $\mathrm{z} / \mathrm{D}=-0.44$ to $\mathrm{z} / \mathrm{D}=0.39$ respectively. The height of the coolant is $\mathrm{y} / \mathrm{D}=0.85$ and spreads from $\mathrm{z} / \mathrm{D}=-0.7$ to $\mathrm{z} / \mathrm{D}=0.67$. Therefore, the lateral spreading of the coolant is more affected by imperfection shape at this location. 


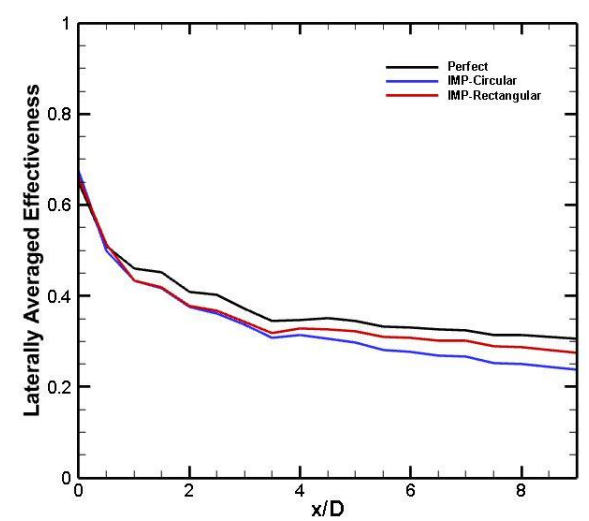

a) $\mathrm{M}=0.45,26.3 \%$

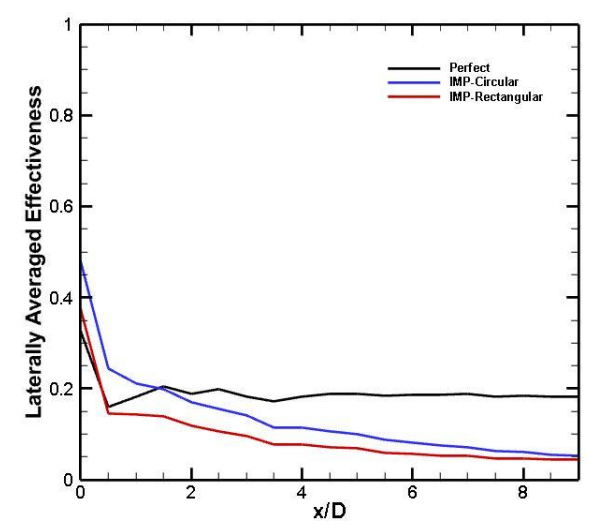

d) $\mathrm{M}=0.9,26.3 \%$

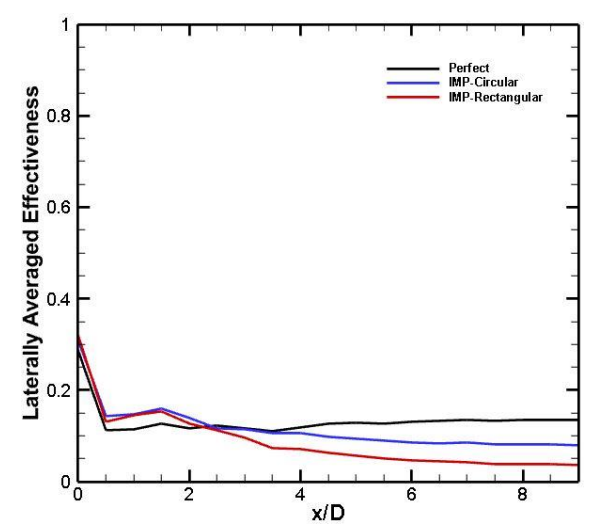

g) $\mathrm{M}=1.25,26.3 \%$

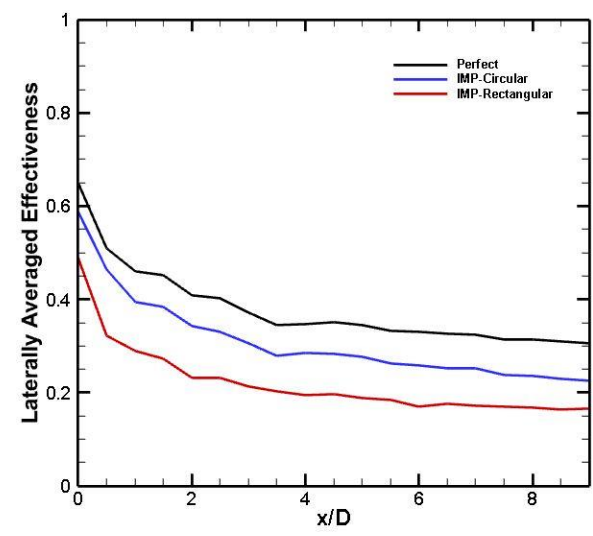

b) $\mathrm{M}=0.45,35 \%$

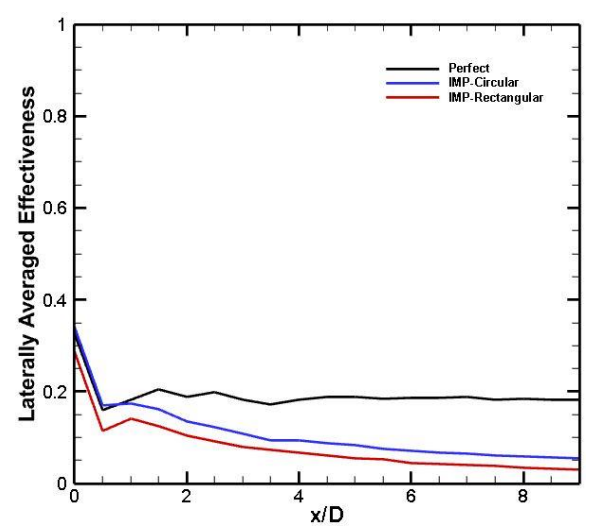

e) $\mathrm{M}=0.9,35 \%$

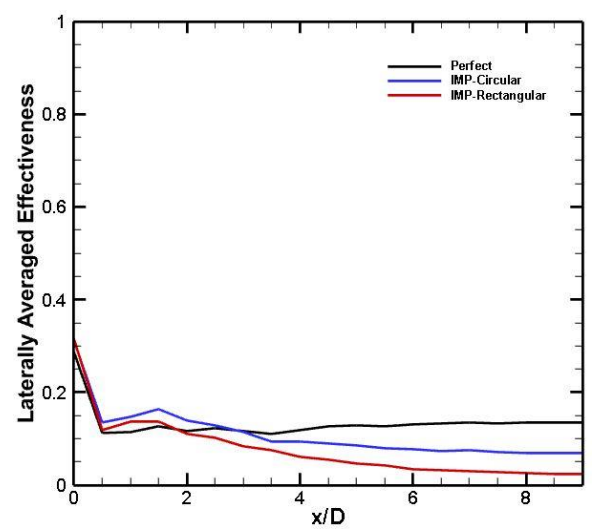

h) $\mathrm{M}=1.25,35 \%$

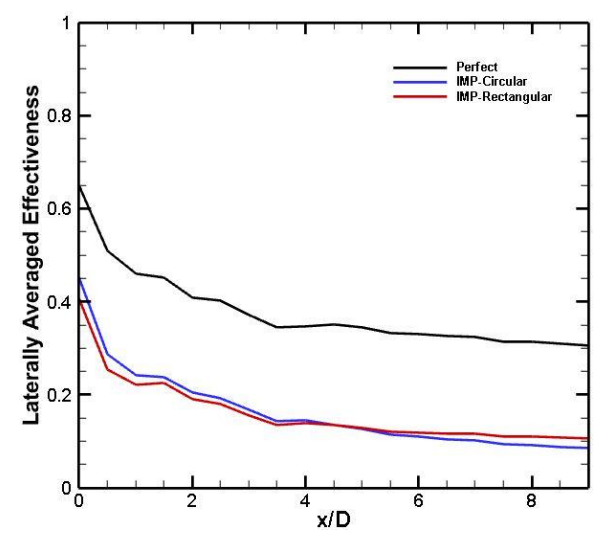

c) $\mathrm{M}=0.45,40 \%$

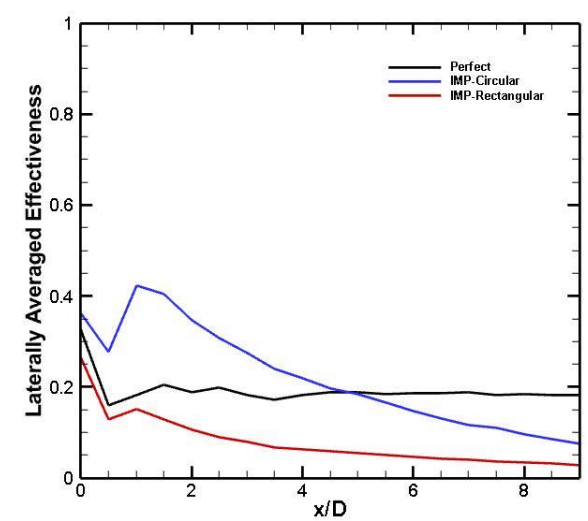

f) $M=0.9,40 \%$

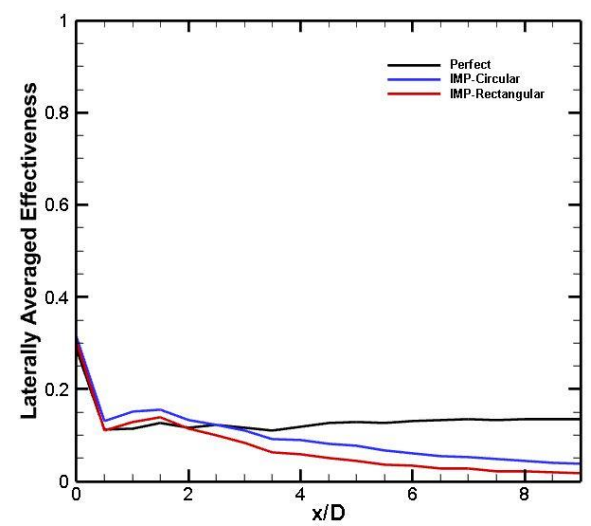

i) $\mathrm{M}=1.25,40 \%$

Figure 27. Laterally Averaged Film Cooling Effectiveness; Perfect (Black), Imperfect Circular (Blue) and Rectangular (Red) Cases 


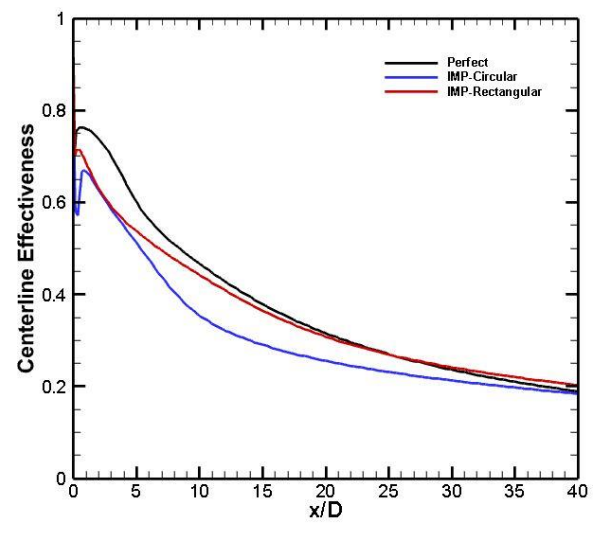

a) $\mathrm{M}=0.45,26.3 \%$

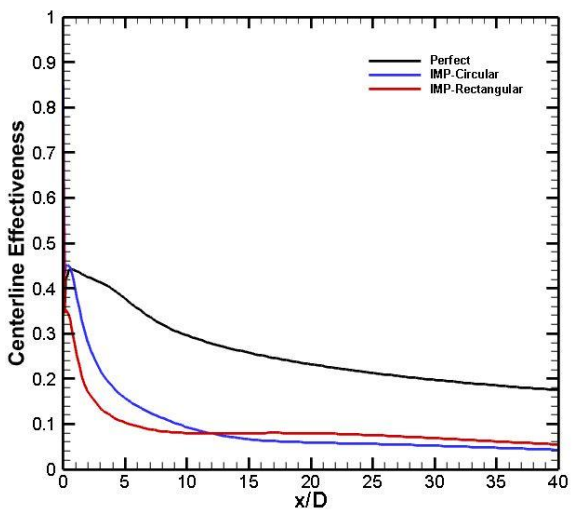

d) $\mathrm{M}=0.9,26.3 \%$

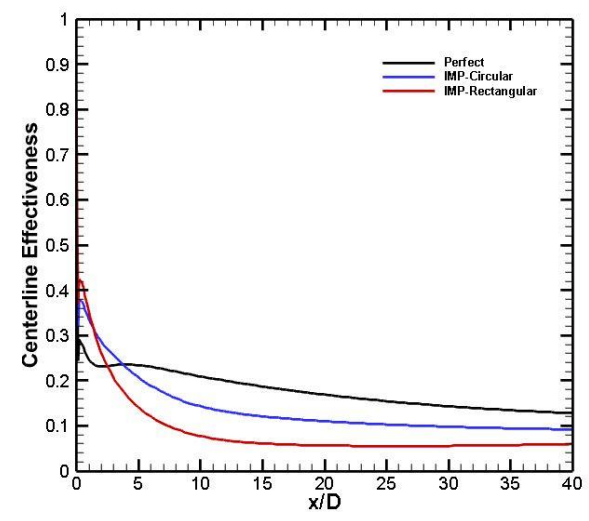

g) $\mathrm{M}=1.25,26.3 \%$

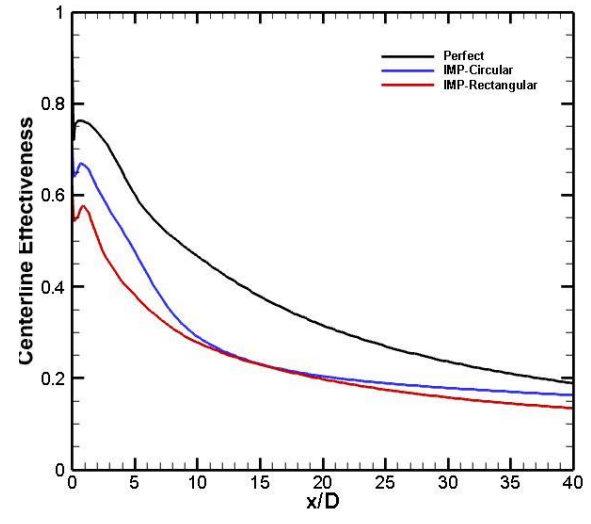

b) $\mathrm{M}=0.45,35 \%$

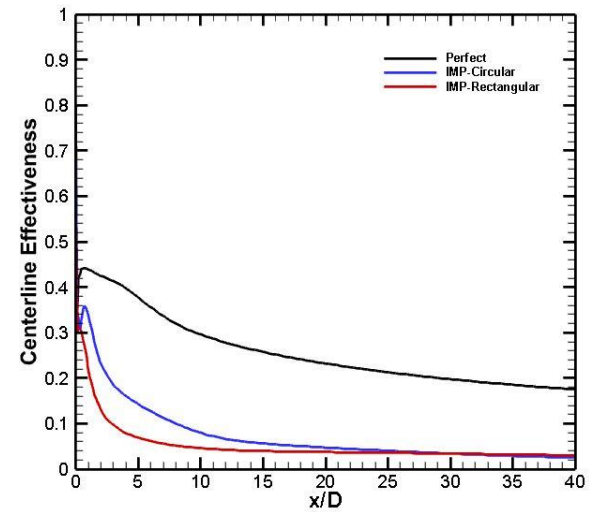

e) $\mathrm{M}=0.9,35 \%$

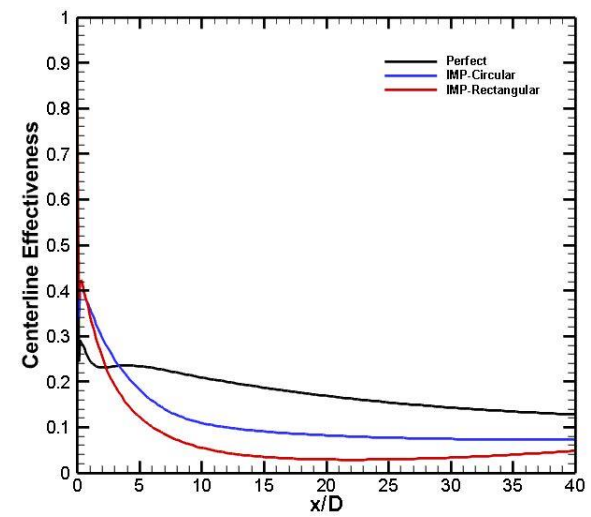

h) $\mathrm{M}=1.25,35 \%$

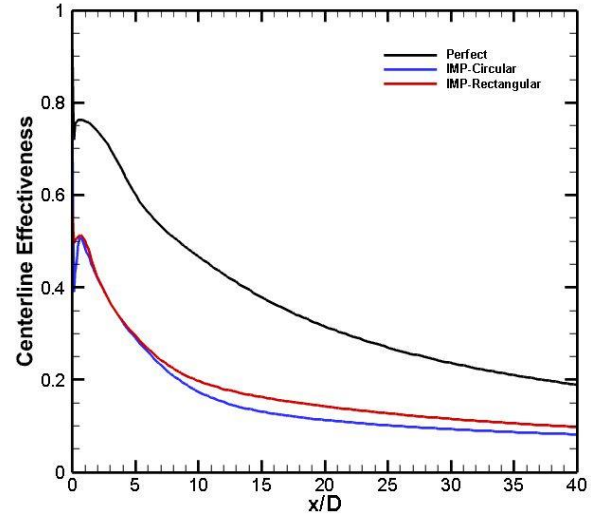

c) $\mathrm{M}=0.45,40 \%$

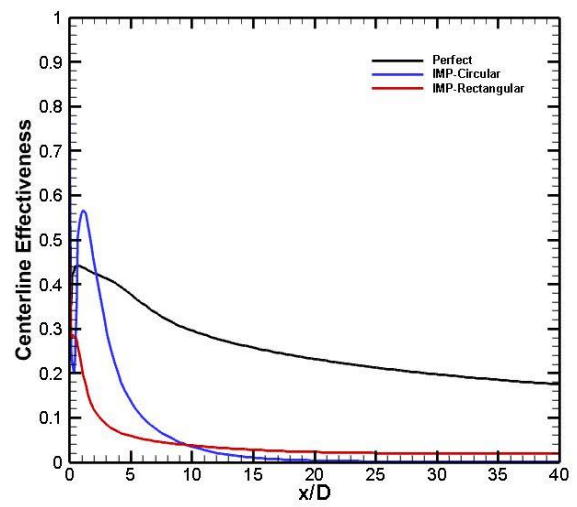

f) $\mathrm{M}=0.9,40 \%$

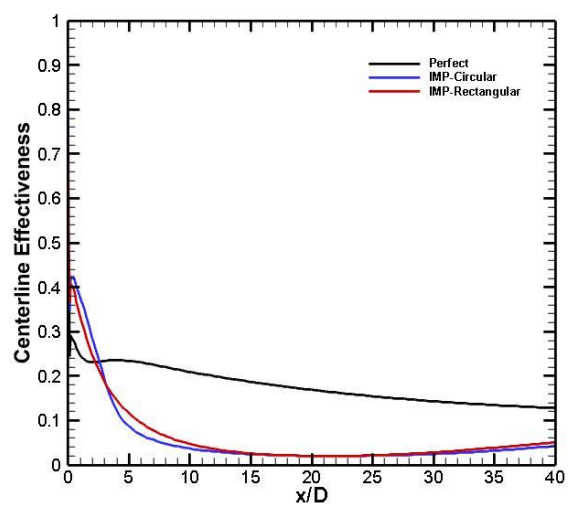

i) $\mathrm{M}=1.25,40 \%$

Figure 28. Centerline Film Cooling Effectiveness; Perfect (Black) and Imperfect Circular (Blue) and Rectangular (Red) Cases 


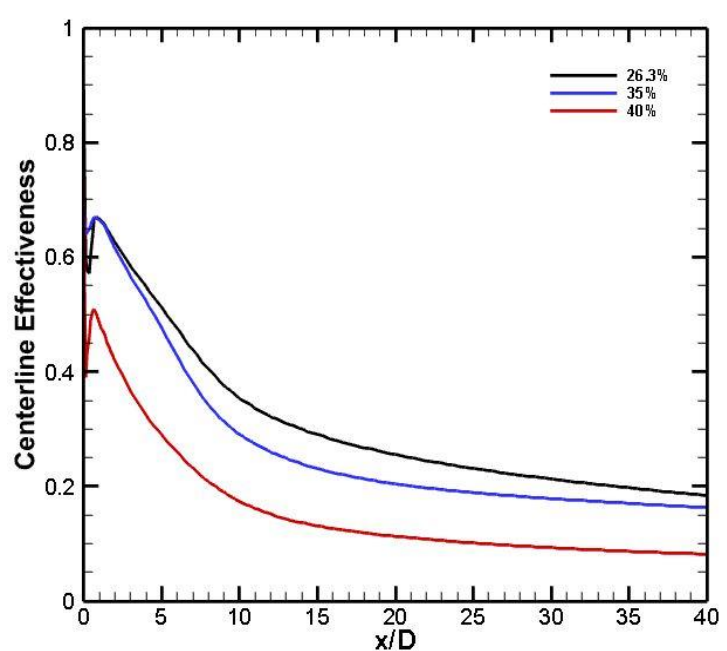

a) Centerline Effectiveness-M=0.45

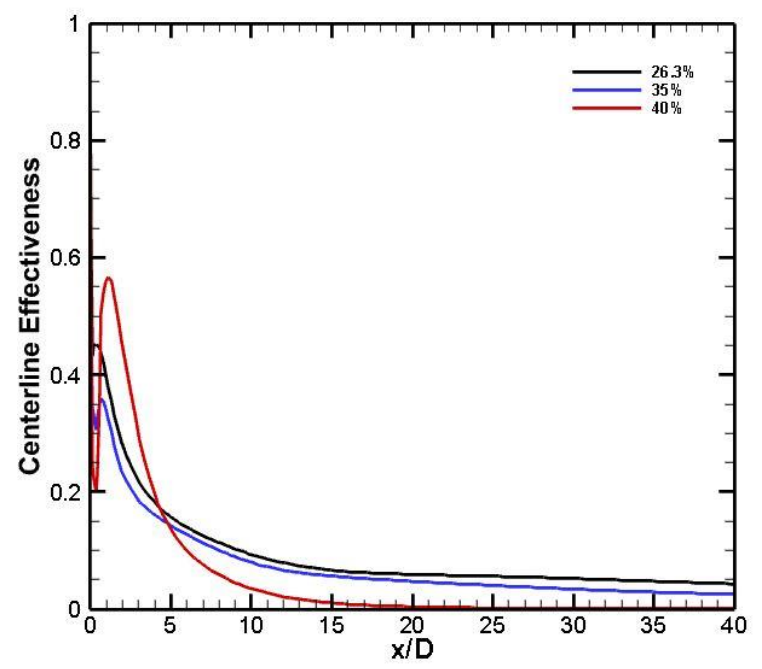

c) Centerline Effectiveness-M=0.90

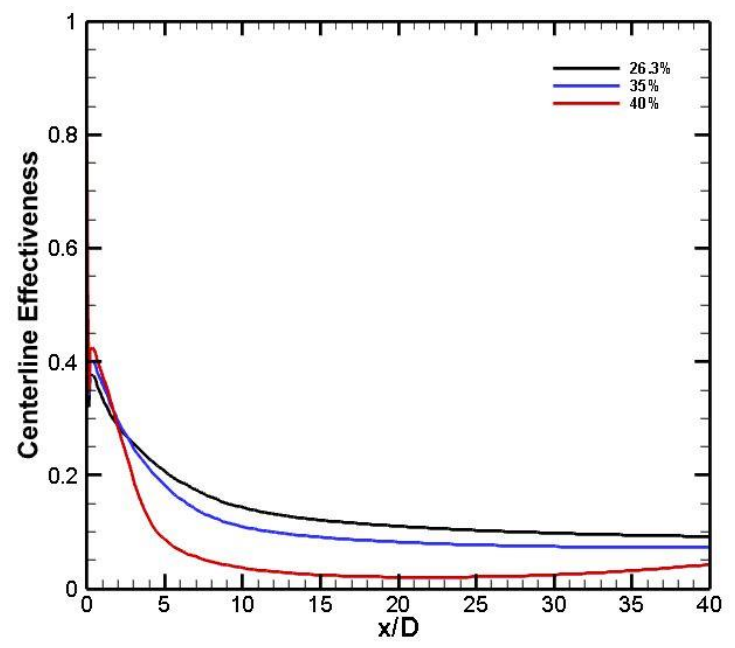

e) Centerline Effectiveness-M=1.25

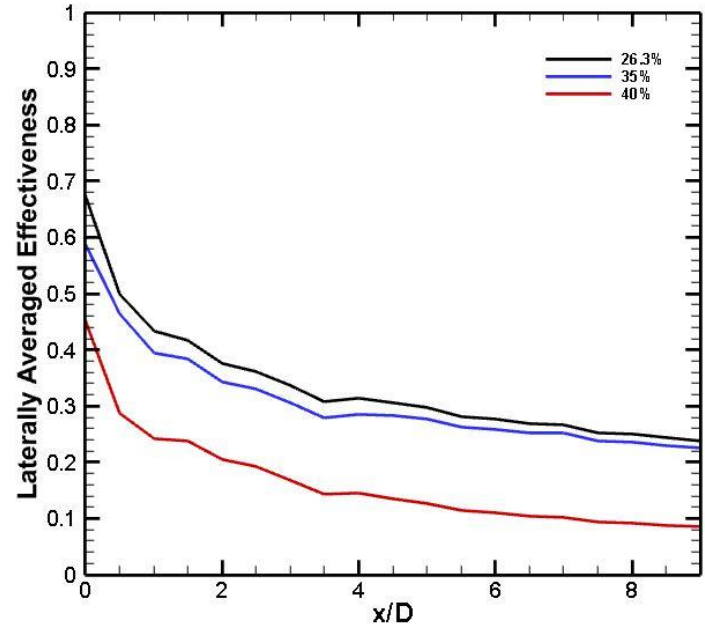

b) Laterally Averaged Effectiveness-M=0.45

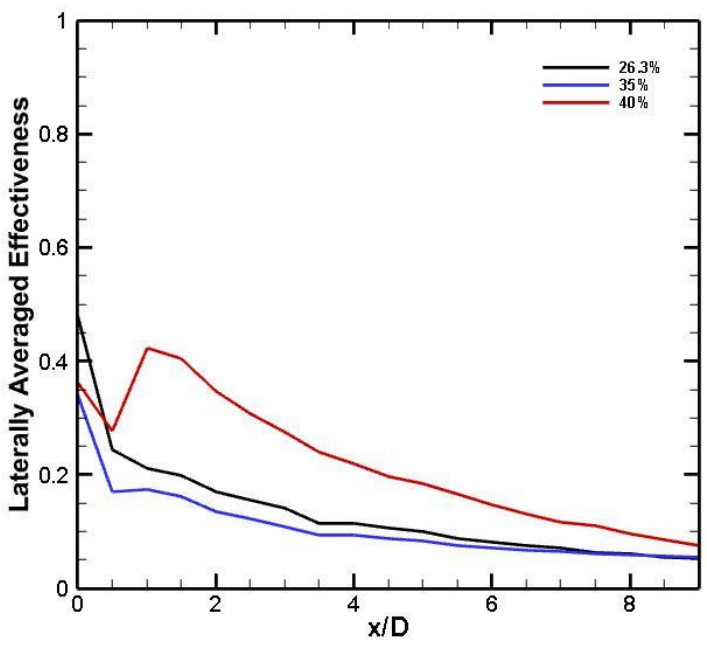

d) Laterally Averaged Effectiveness-M=0.90

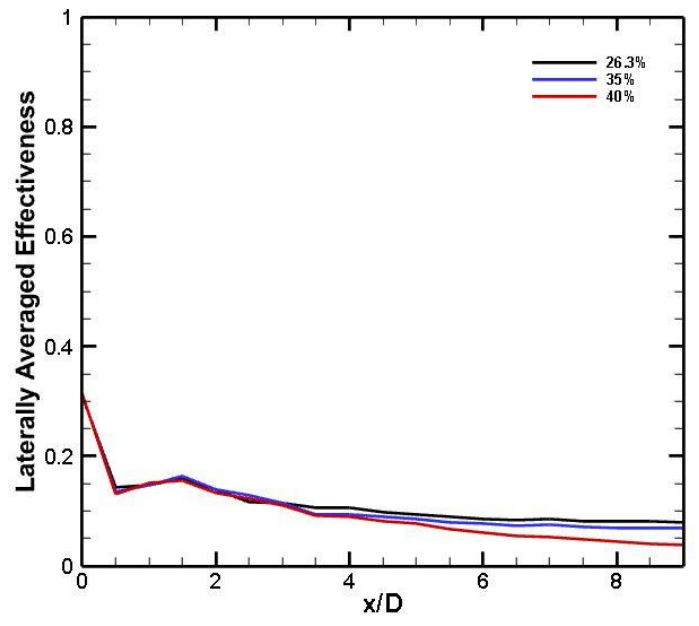

f) Laterally Averaged Effectiveness-M=1.25

Figure 29. Centerline and Laterally Averaged Film Cooling Effectiveness for Circular Imperfection; $26.3 \%$ (Black), 35\% (Blue) and 40\% (Red) 


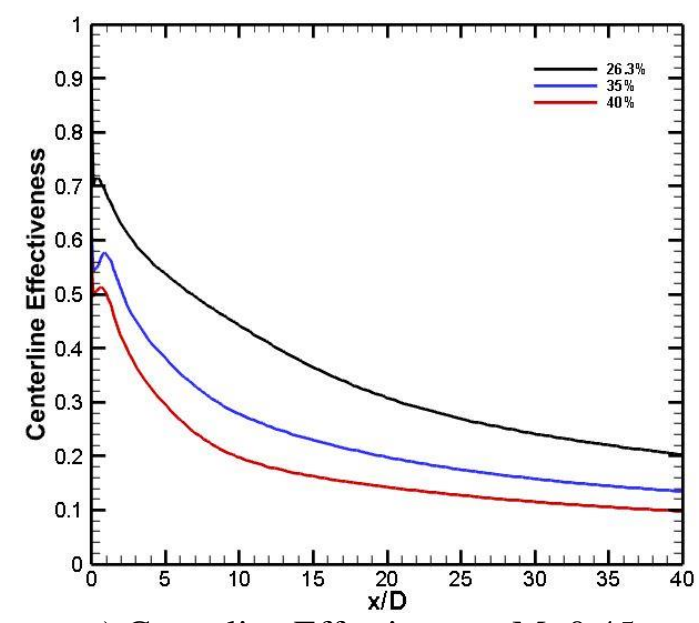

a) Centerline Effectiveness-M=0.45

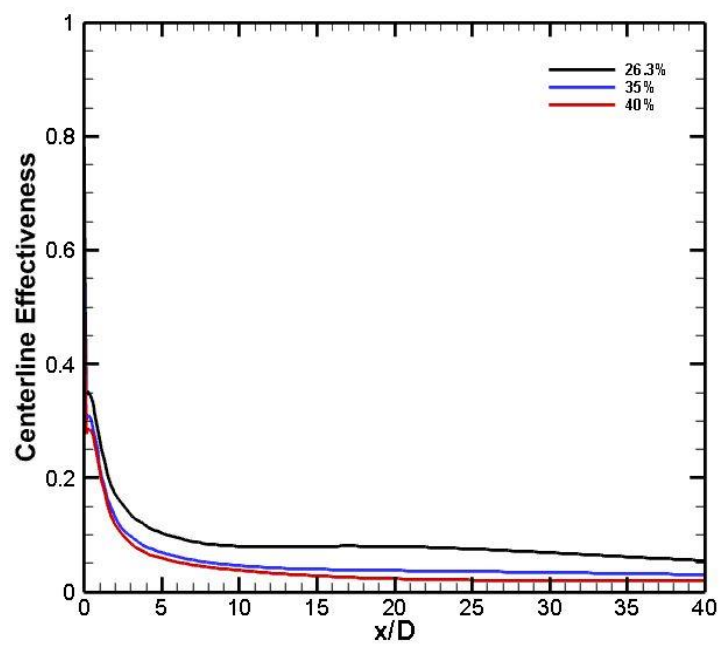

c) Centerline Effectiveness-M=0.90

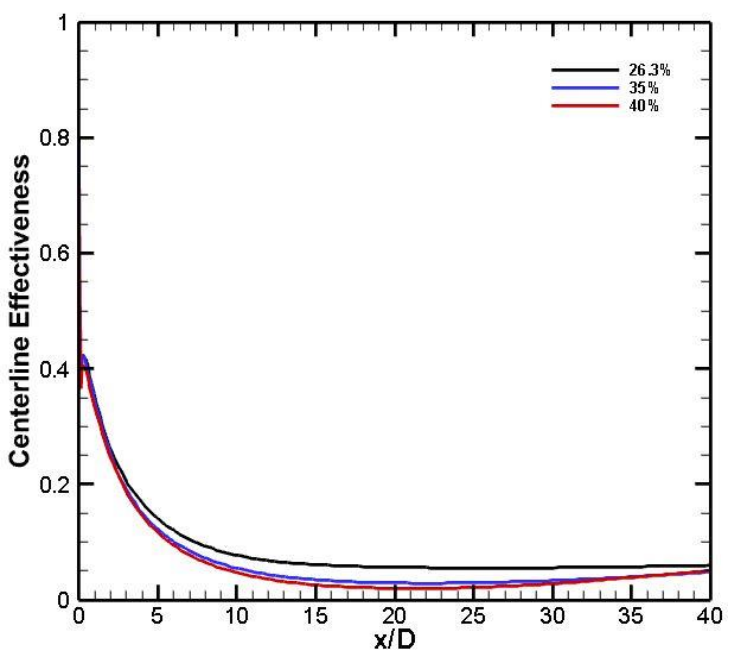

e) Centerline Effectiveness-M=1.25

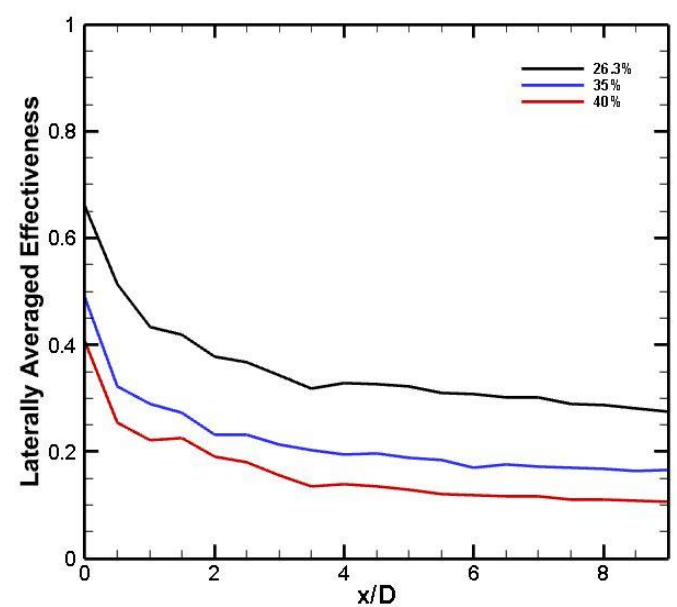

b) Laterally Averaged Effectiveness-M=0.45

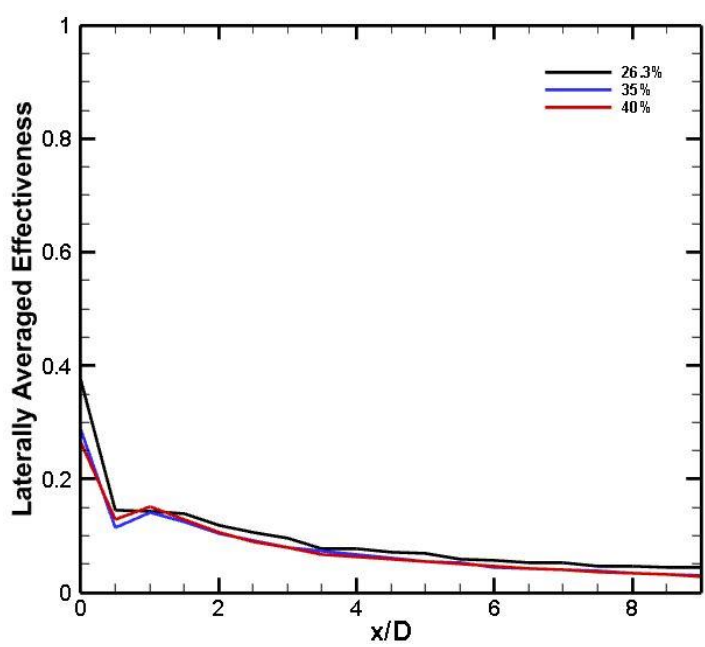

d) Laterally Averaged Effectiveness-M=0.90

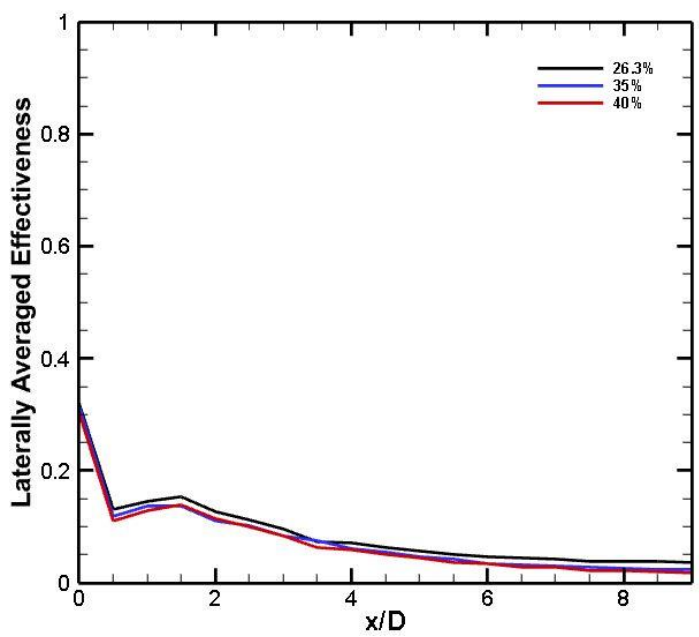

f) Laterally Averaged Effectiveness-M=1.25

Figure 30. Centerline and Laterally Averaged Film Cooling Effectiveness for Rectangular Imperfection; $26.3 \%$ (Black), 35\% (Blue) and 40\% (Red) 


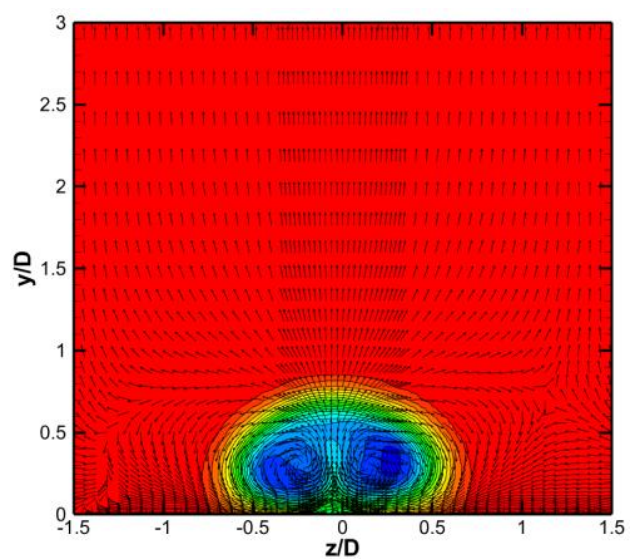

a) Circular- $26.3 \%$

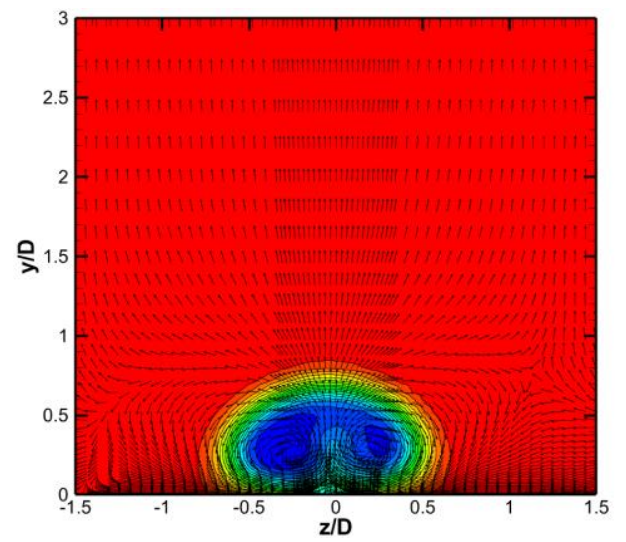

c) Circular-35\%

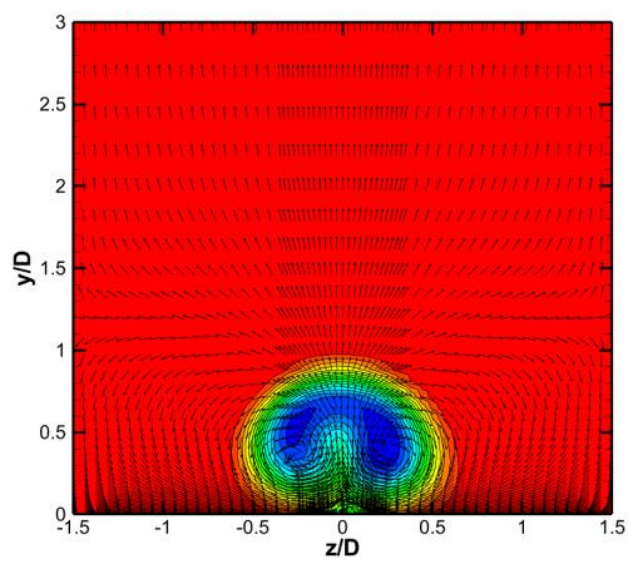

e) Circular- $40 \%$

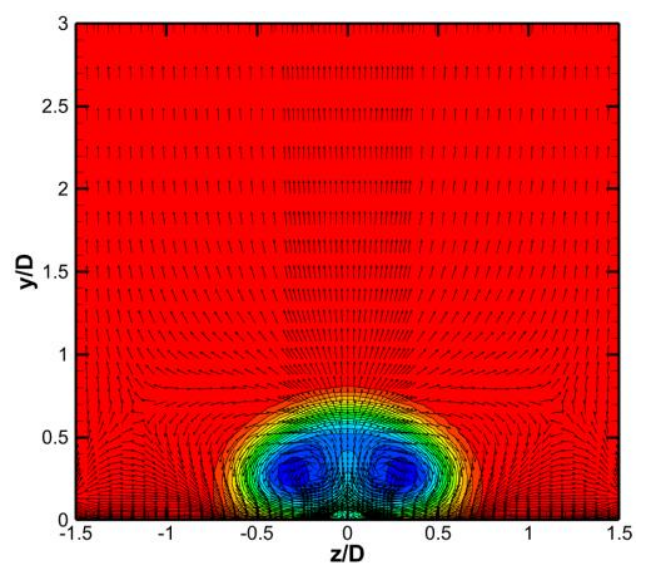

b) Rectangular- $26.3 \%$

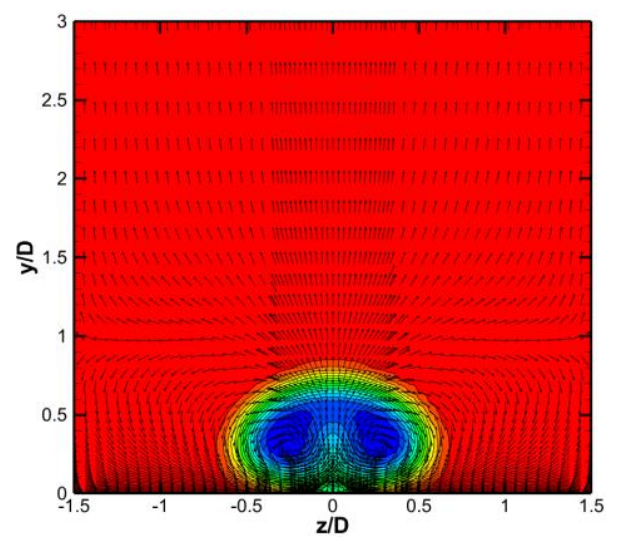

d) Rectangular-35\%

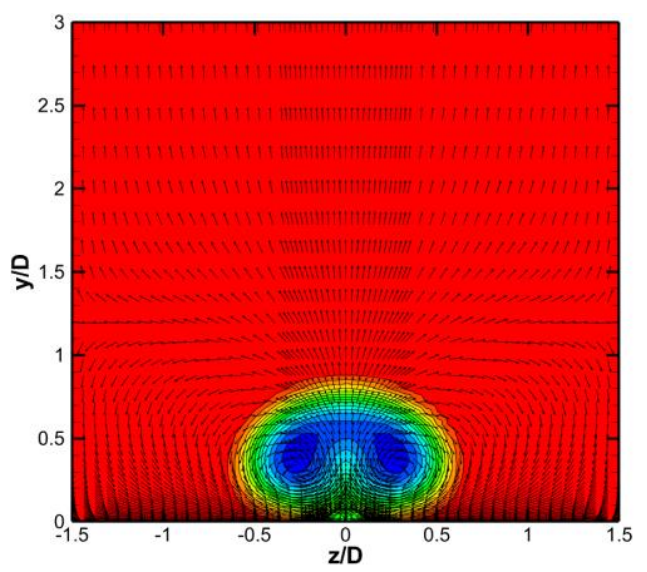

f) Rectangular- $40 \%$

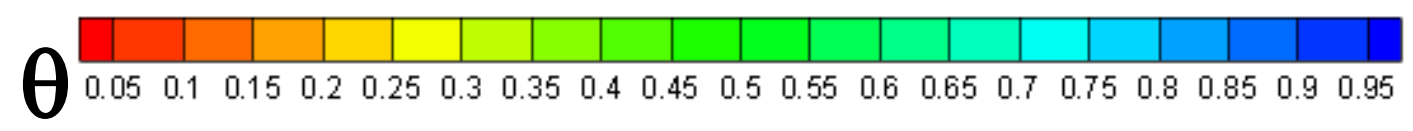

Figure 31. Dimensionless Temperature Contours and Velocity Vectors at $\mathrm{x} / \mathrm{D}=2$ for $\mathrm{M}=0.45$ for Circular (left) and Rectangular (right) Shaped Imperfections at Various Sizes 


\section{CONCLUSIONS AND FUTURE RECOMMENDATIONS}

The effects of hole imperfections, due to laser percussion drilling, on film cooling performance over a flat plate were numerically investigated. Various turbulence models combined with wall functions and wall treatments were studied in order to find the appropriate combination capable of providing the best prediction of both laterally averaged and centerline film cooling effectiveness for the imperfection. It was found that the realizable k-epsilon turbulence model with the enhanced wall treatment gave results that agreed well for all blowing ratios with experimental data for the perfect case. Moreover, the SST k-omega turbulence model agreed well with experimental data for blowing ratios of $\mathrm{M}=0.45$ and $\mathrm{M}=0.90$, whereas the realizable k-epsilon model with the enhanced wall treatment was chosen for a blowing ratio of $M=1.25$ for the imperfect case.

Results showed that the position of the imperfection inside the hole has an effect on the jet structure and flow field thus affecting film cooling effectiveness. Laterally averaged and centerline effectiveness plots indicate that as the imperfection is placed farther inside the hole, a significant improvement in effectiveness is achieved. For instance, when the imperfection is located 4D from the hole exit for a blowing ratio of $\mathrm{M}=0.45$, a $46.5 \%$ improvement in laterally averaged effectiveness at $\mathrm{x} / \mathrm{D}=1$ is achieved. Dimensionless temperature contour reveal that jet emerging from hole with imperfections farther from the hole exit stay much closer to the surface of the plate.

Results showed that hole inclination angle with the presence of an imperfection located 1D inside influences the flow field in the vicinity of the hole exit which affects the film cooling performance. Analysis of the centerline effectiveness plots revealed that the hole imperfection caused deterioration in centerline effectiveness for all blowing ratios and hole inclination angles compared to the perfect case. Furthermore, it was found that hole inclination angle had more influence on the imperfection case than the baseline case. The centerline effectiveness was found to decrease considerably as the hole inclination angle is increased. It was observed that a maximum centerline effectiveness deterioration of $89 \%$ occurred at $\mathrm{x} / \mathrm{D}=1$ at a blowing ratio of 0.90 with a hole inclination angle of $55^{\circ}$. The dimensionless temperature contours showed that coolant jets formed through the hole imperfection were much more compact than those from the baseline case. This causes the counter rotating vortex pairs to be closer to each other enhancing jet lift off. Finally, results showed that the shape and size of the imperfection inside the hole has an effect on the jet structure and flow field thus affecting film cooling effectiveness. Laterally averaged and centerline effectiveness plots indicate that as the imperfection size increases, the deterioration in film cooling performance decreases significantly. For instance, it can be seen at $\mathrm{x} / \mathrm{D}=10$ for a blowing ratio of $\mathrm{M}=0.9$ that a deterioration of $262.5 \%, 533.2 \%$ and $735.7 \%$ in centerline effectiveness compared the perfect case at $26.3 \%, 35 \%$ and $40 \%$ obstructions respectively. Dimensionless temperature contour reveal that the lateral 
spreading of the coolant is more affected by imperfection shape at the location of $x / D=2$ where the circular shaped imperfection provides better laterally averaged effectiveness than the rectangular shaped imperfection especially of the $35 \%$ obstruction size.

Investigations on the effects of hole imperfections, due to laser percussion drilling, on film cooling performance are far from being completed and this work offers a platform where future studies can be built upon. Here are a few suggestions:

- Effect of pitch to diameter ratio

- Effect of freestream turbulence intensity

- Effect of imperfections in shaped holes

- Effect of imperfections with hole compound angles

- Effect of imperfections inside sister holes and anti-vortex holes

- Effect of hole imperfections inside trenches

- Effect of hole imperfections on turbine blade such as the AGBT 


\section{APPENDIX- TURBULENCE MODELING RESULTS}

\section{Perfect Case}

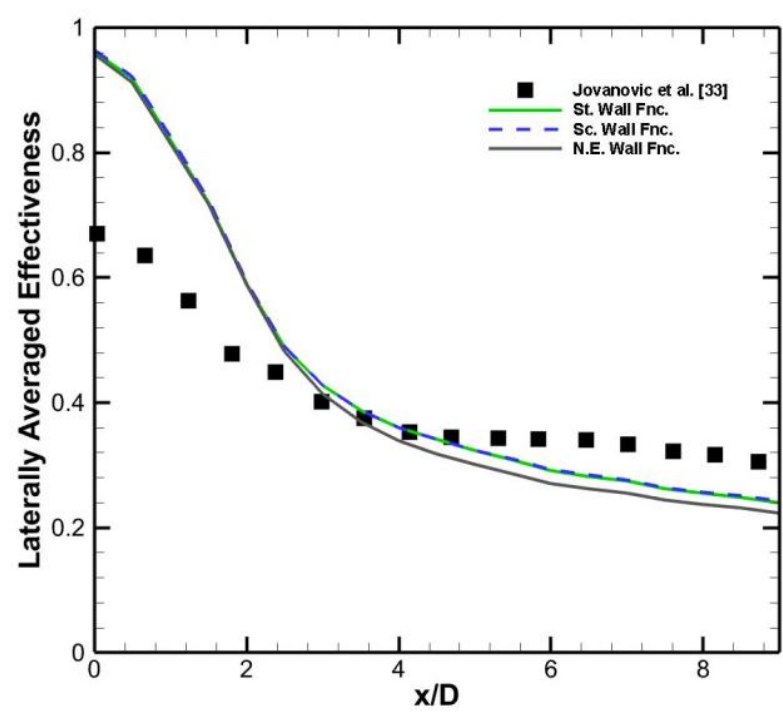

a) St. k-epsilon

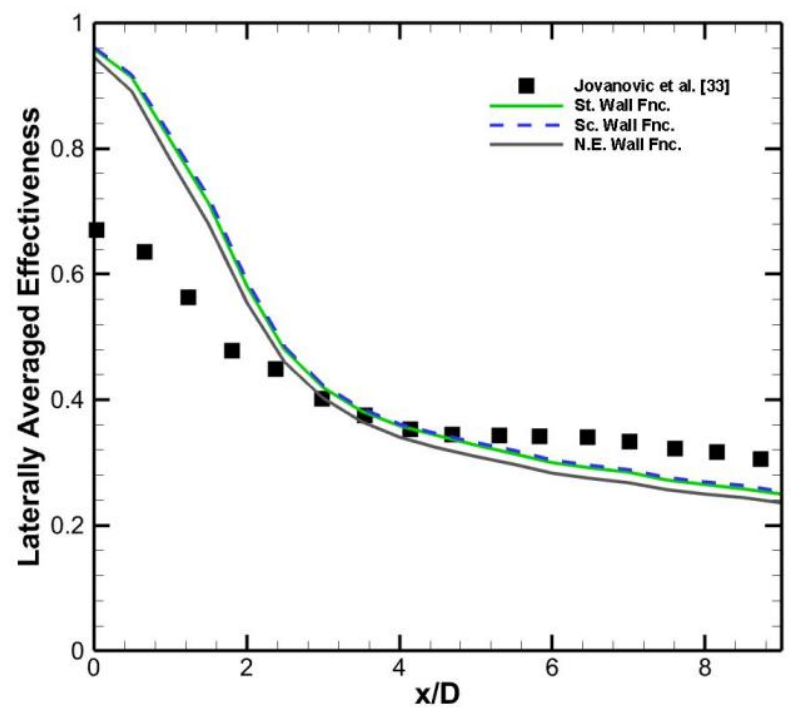

c) Realizable k-epsilon

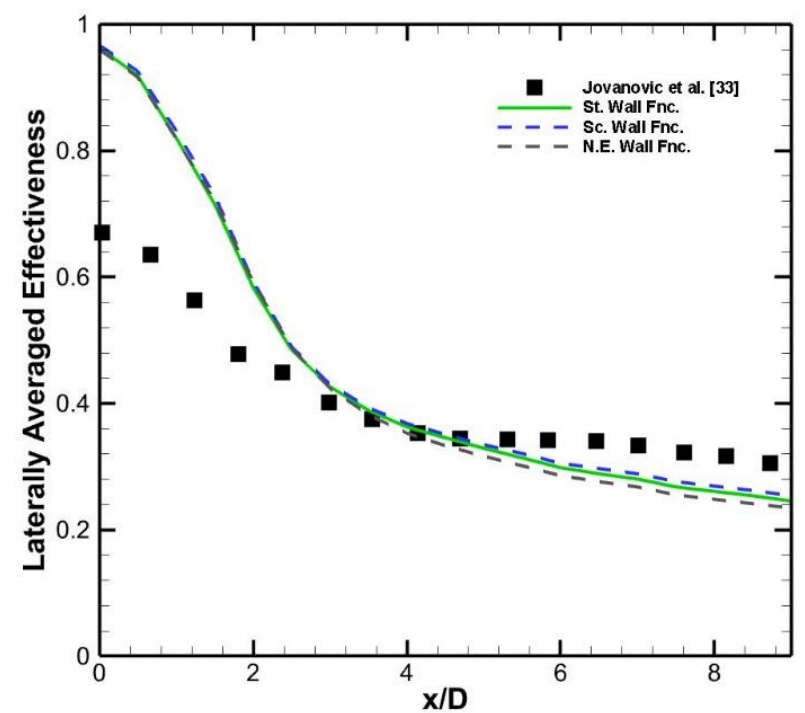

b) RNG k-epsilon

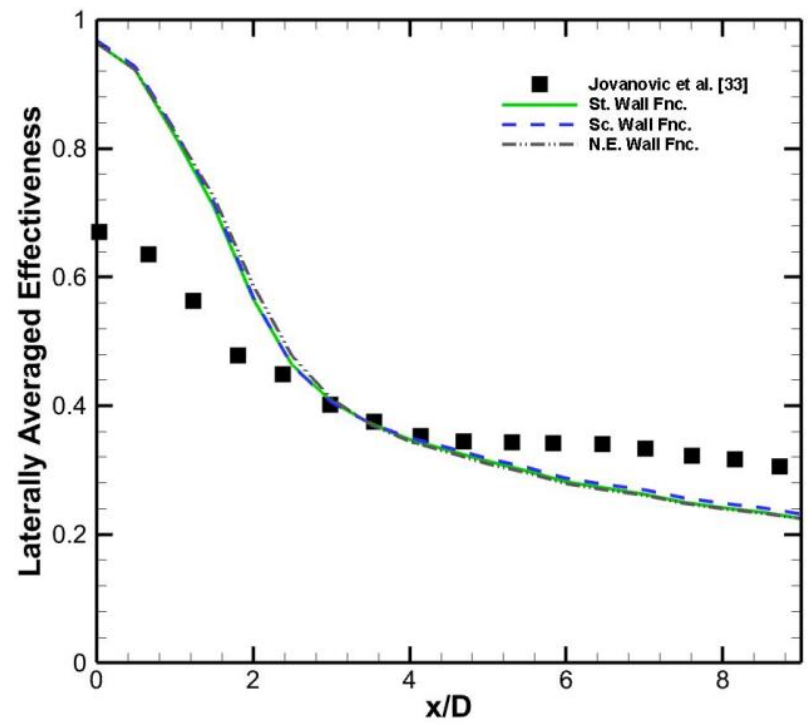

d) Reynolds Stress

Figure 32. Laterally Averaged Film Cooling Effectiveness at a Blowing Ratio of 0.45 and Density Ratio of 1 at $\mathrm{Y}+$ of 30 for the Perfect Case 


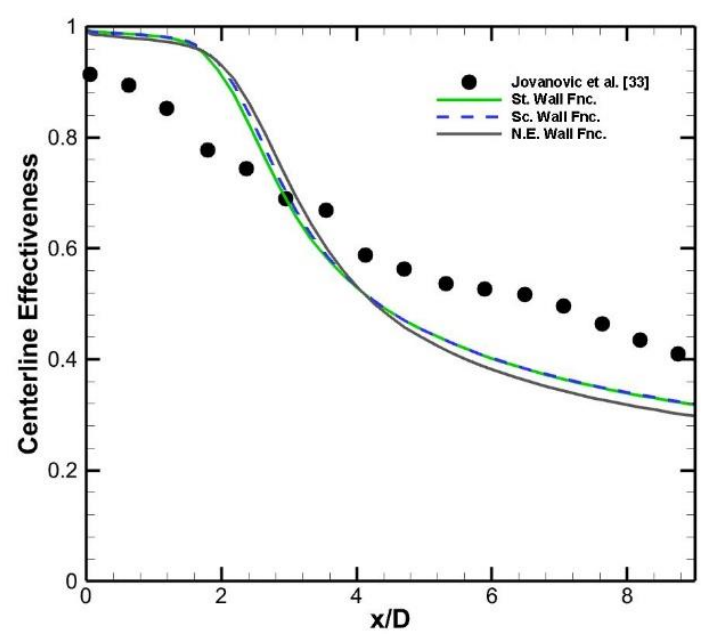

a) St. k-epsilon

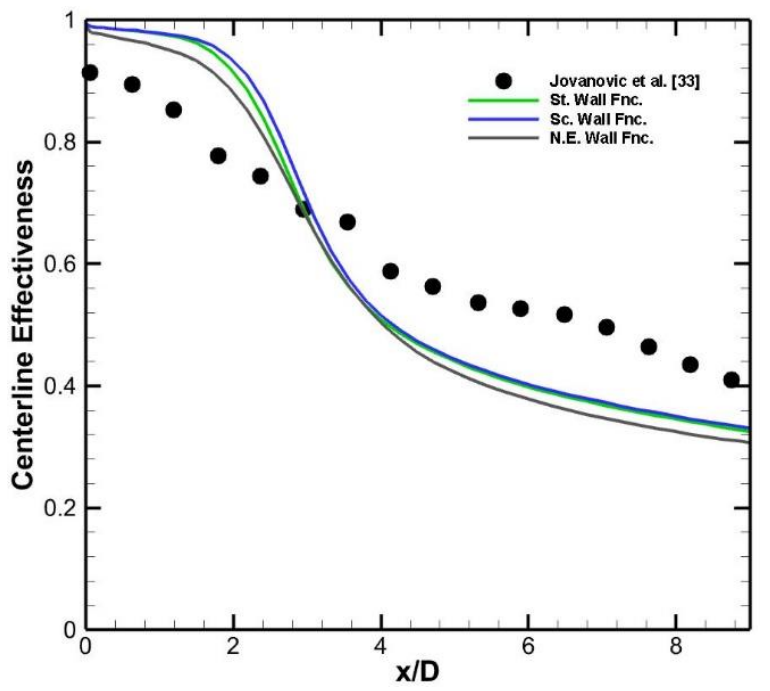

c) Realizable k-epsilon

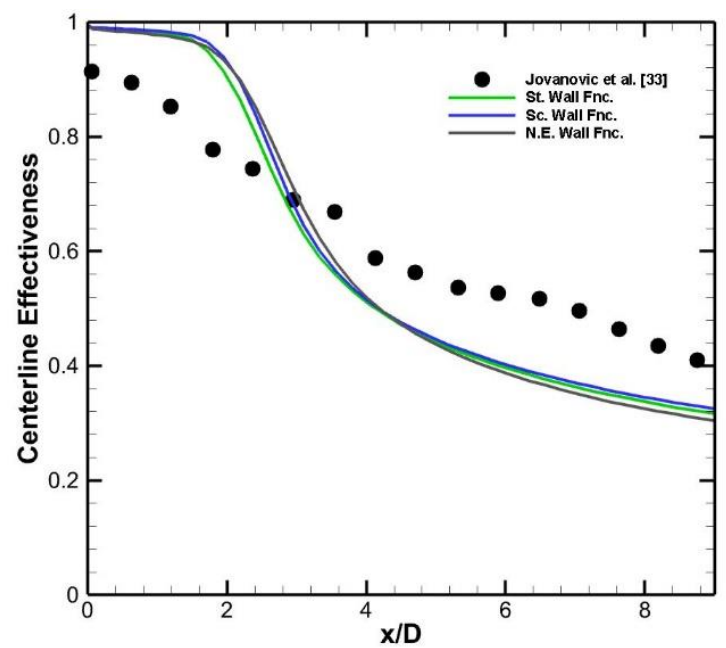

b) RNG k-epsilon

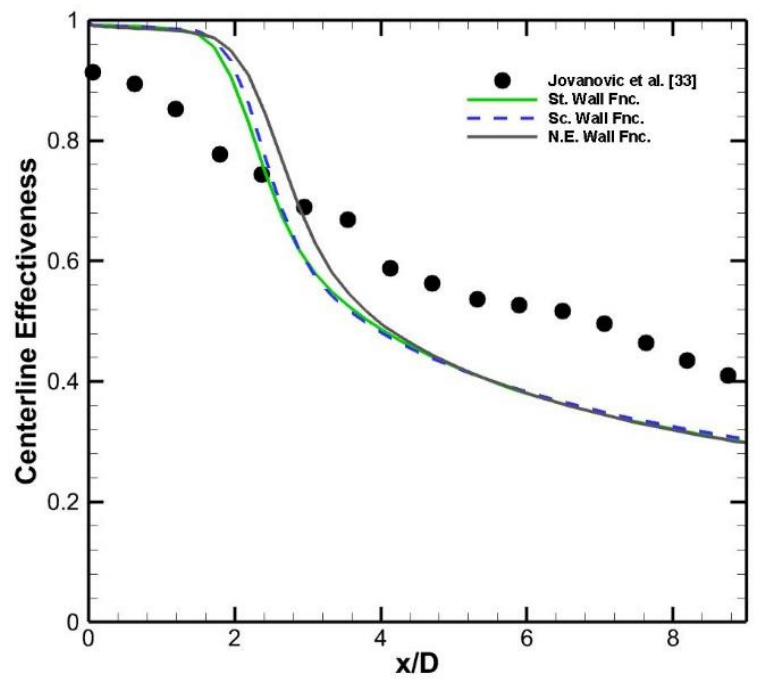

d) Reynolds Stress

Figure 33. Centerline Film Cooling Effectiveness at a Blowing Ratio of 0.45 and Density Ratio of 1 at $\mathrm{Y}+$ of 30 for the Perfect Case 


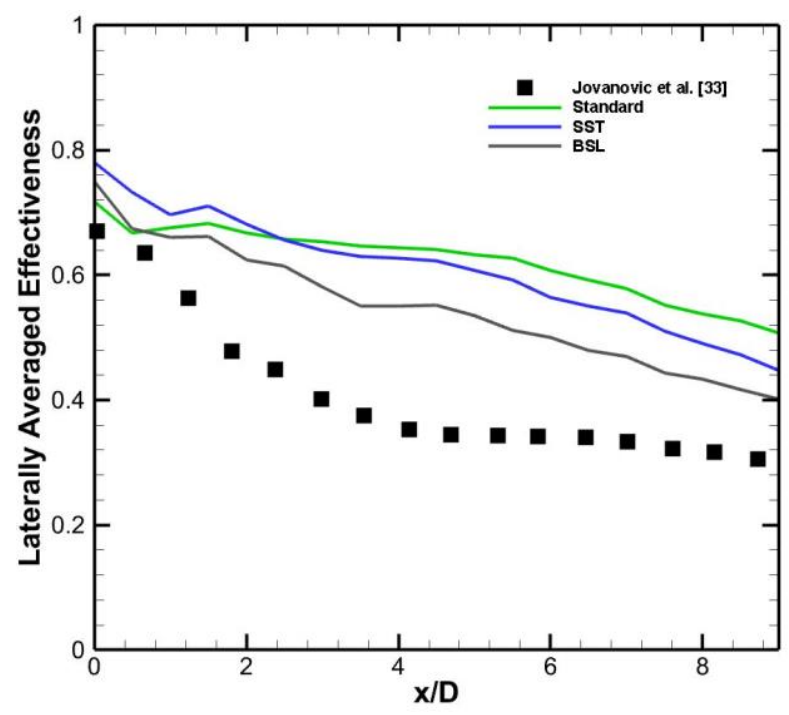

a) k-omega, laterally averaged effectiveness

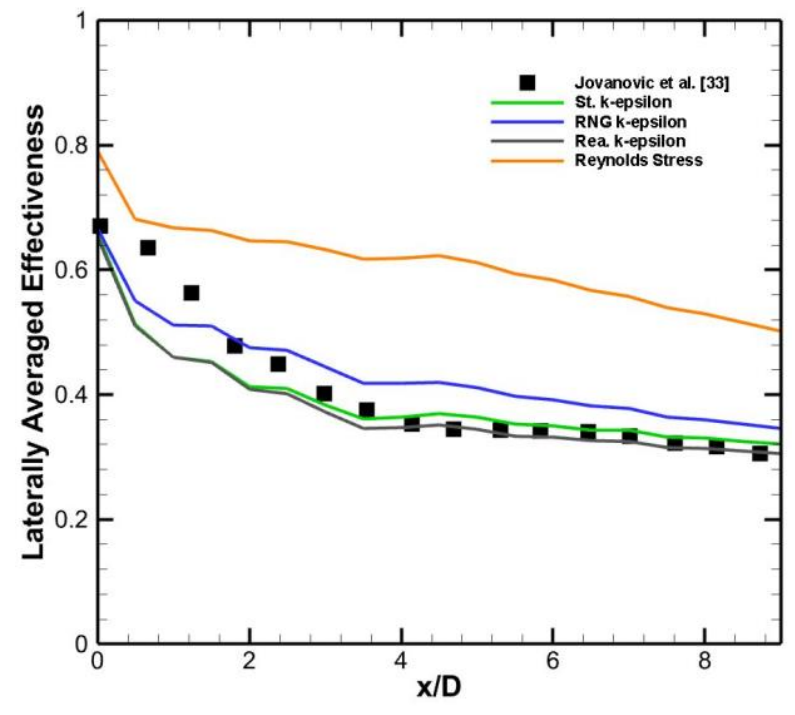

c) k-epsilon, laterally averaged effectiveness

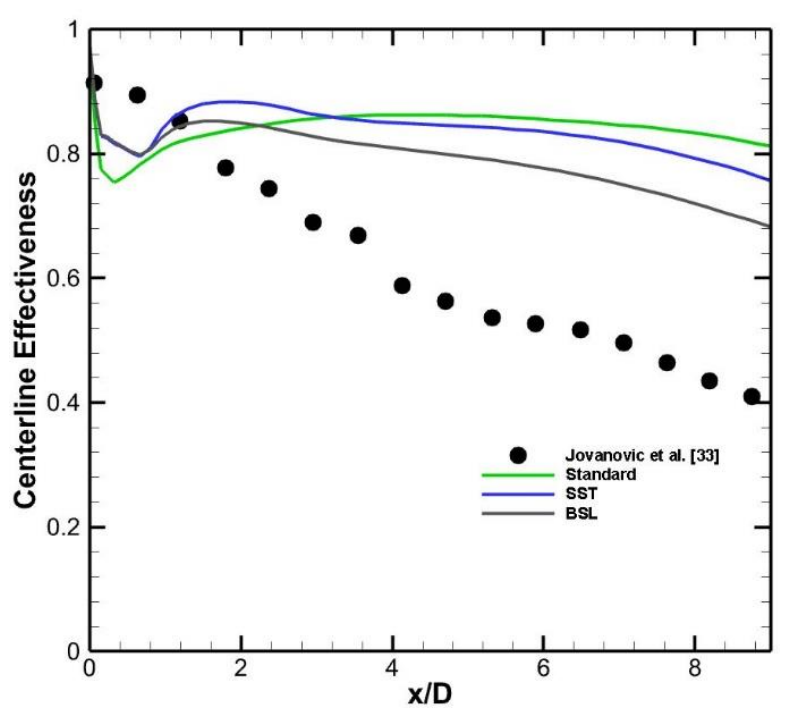

b) k-omega, centerline effectiveness

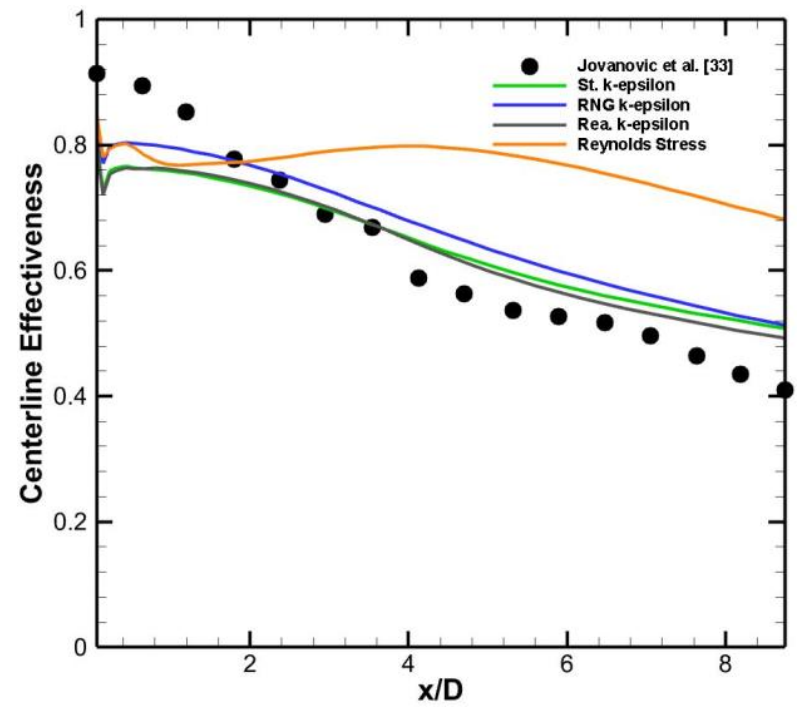

d) k-epsilon, centerline effectiveness

Figure 34. Film Cooling Effectiveness at a Blowing Ratio of 0.45 and Density Ratio of 1 at $\mathrm{Y}+$ of 1 for the Perfect Case 


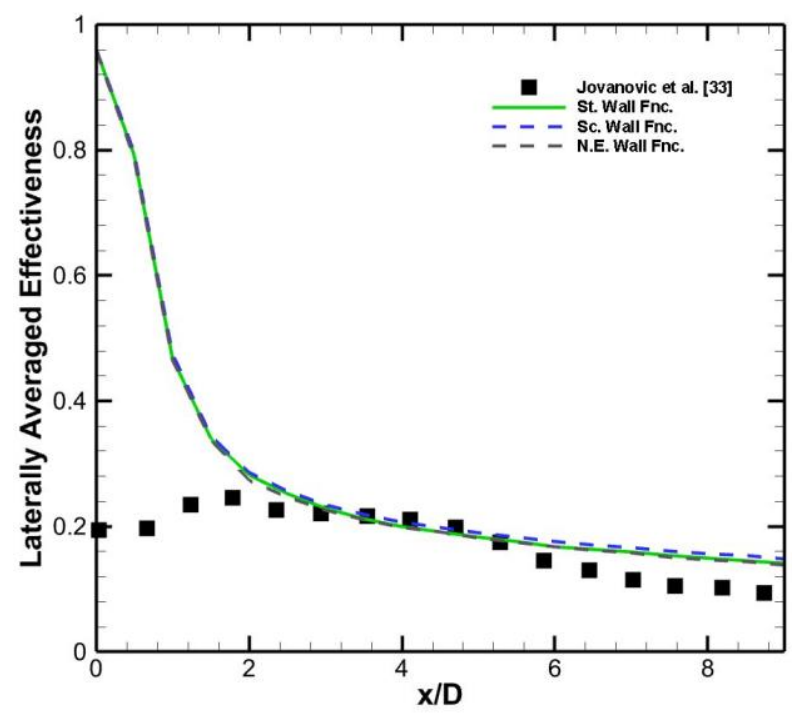

a) St. k-epsilon

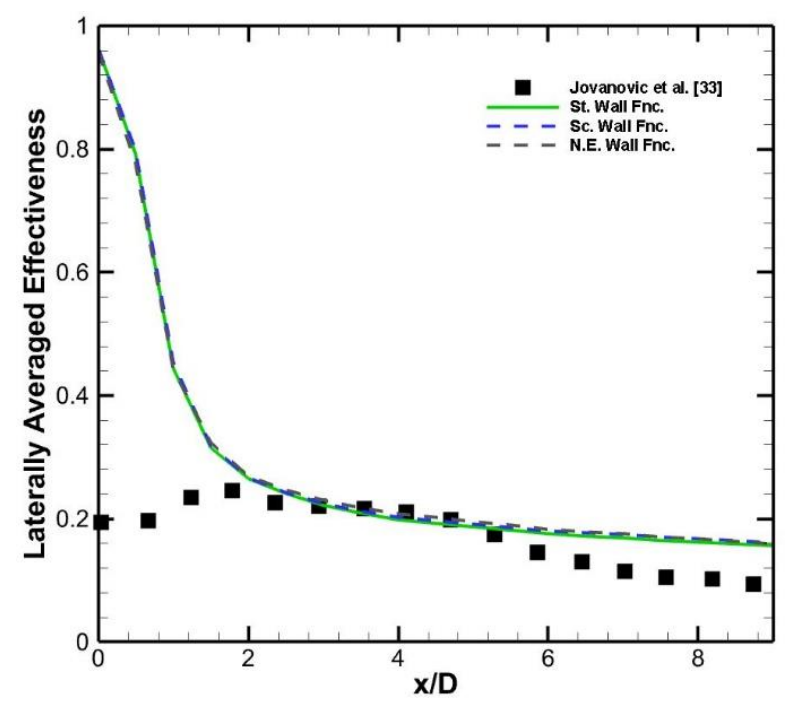

c) Realizable k-epsilon

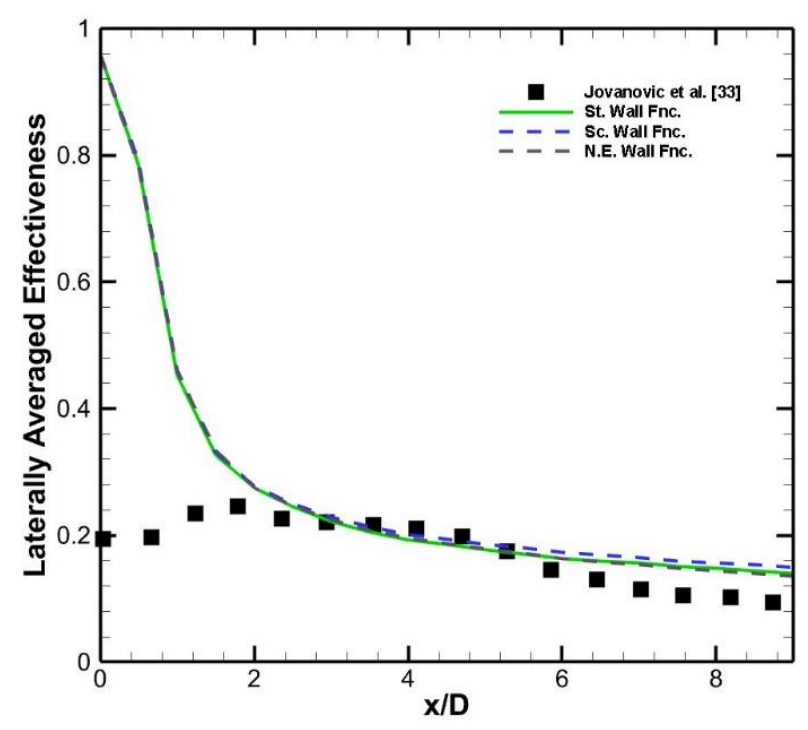

b) RNG k-epsilon

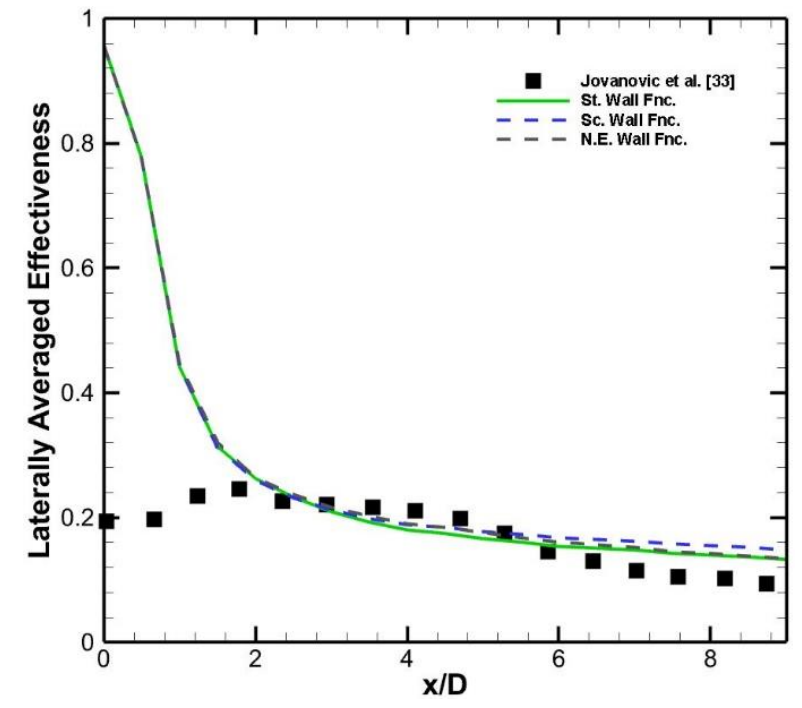

d) Reynolds Stress

Figure 35. Laterally Averaged Film Cooling Effectiveness at a Blowing Ratio of 0.90 and Density Ratio of 1 at $\mathrm{Y}+$ of 30 for the Perfect Case 


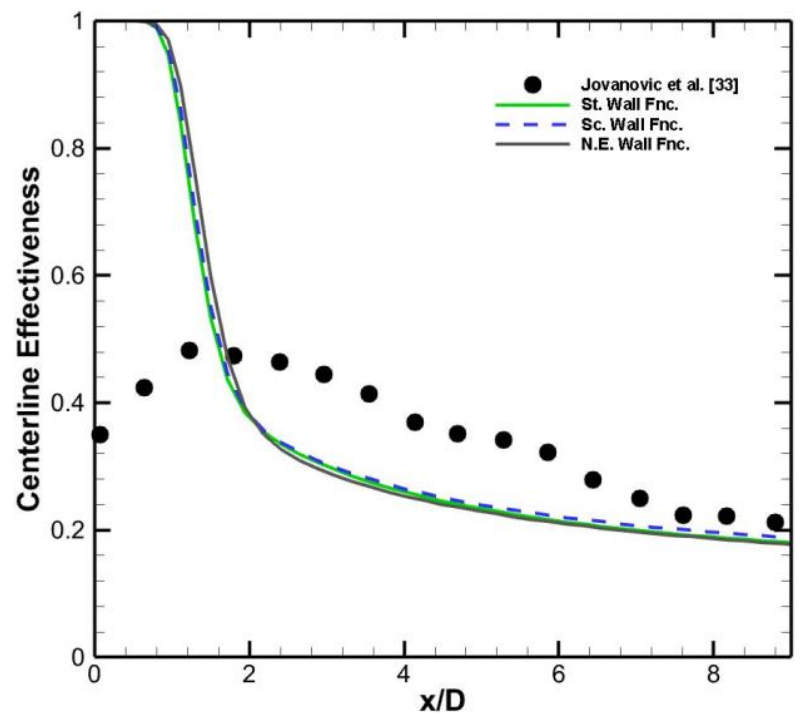

a) St. k-epsilon

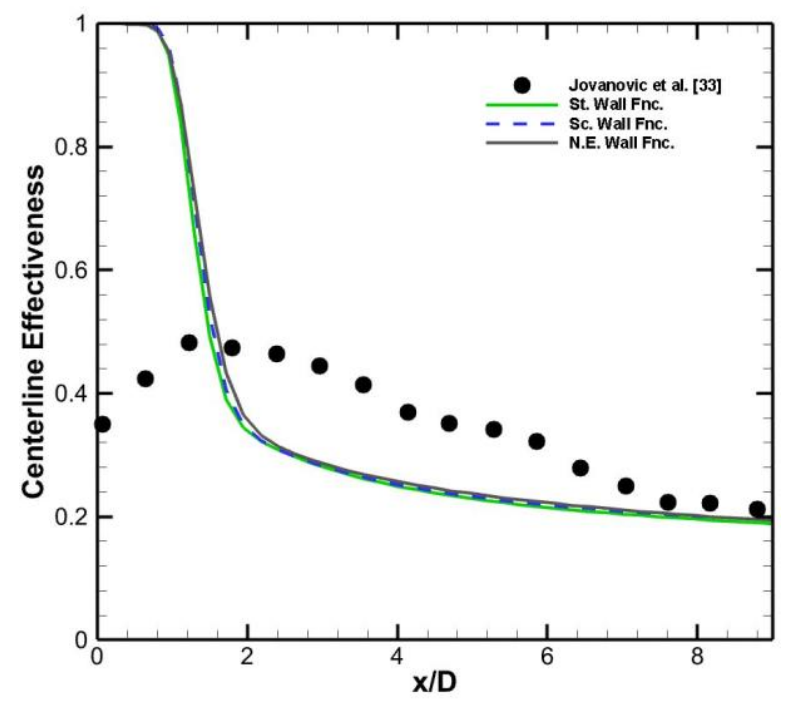

c) Realizable k-epsilon

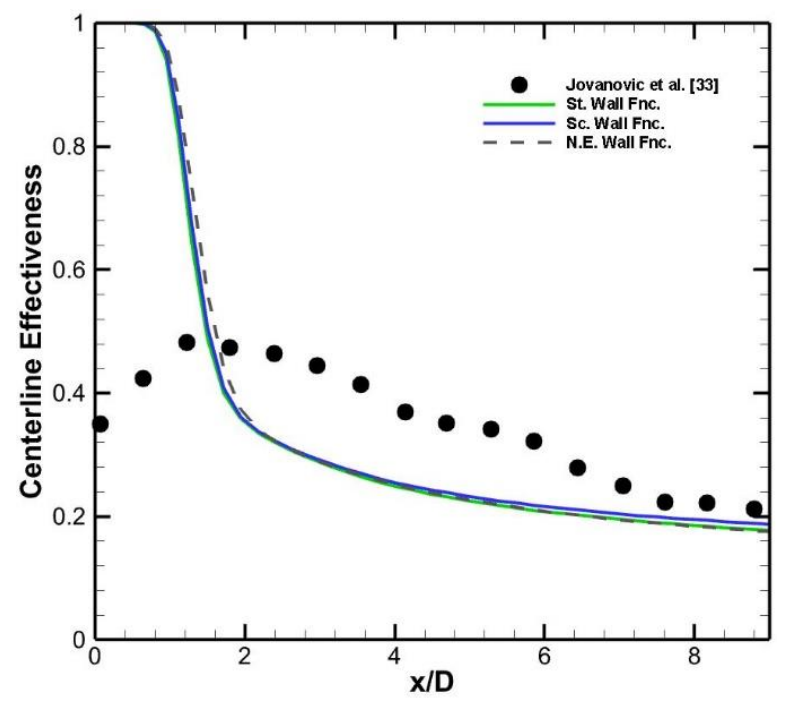

b) RNG k-epsilon

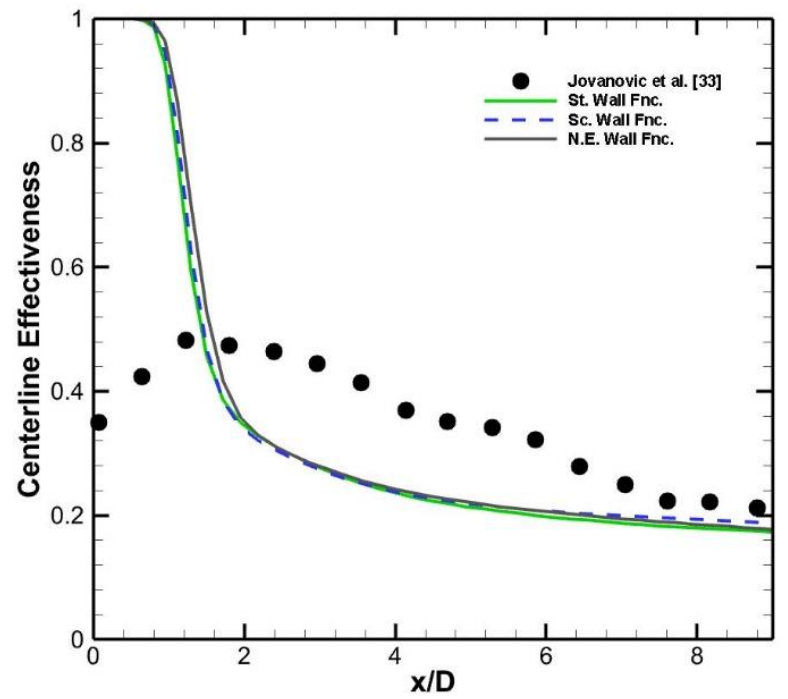

d) Reynolds Stress

Figure 36. Centerline Film Cooling Effectiveness at a Blowing Ratio of 0.90 and Density Ratio of 1 at $\mathrm{Y}+$ of 30 for the Perfect Case 


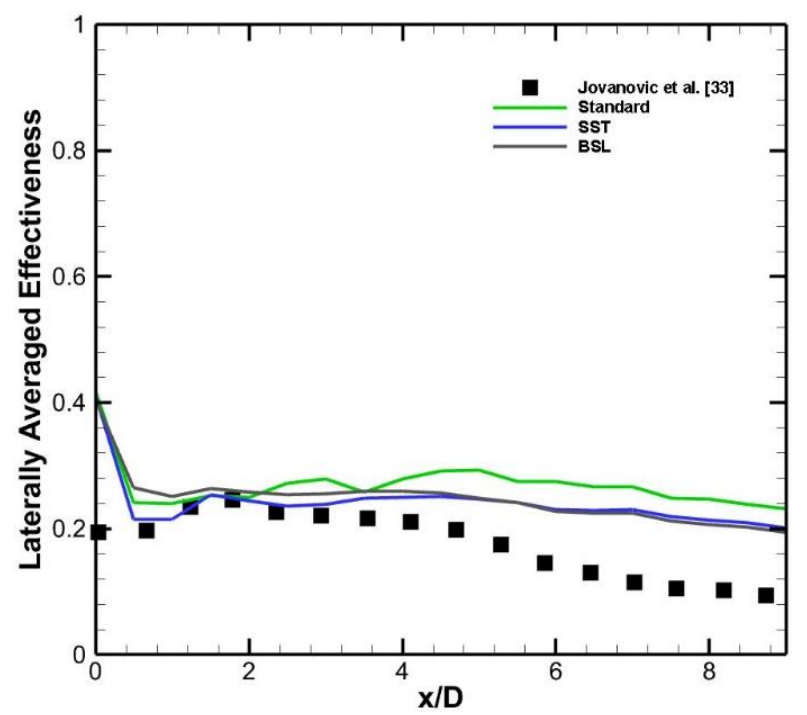

a) k-omega, laterally averaged effectiveness

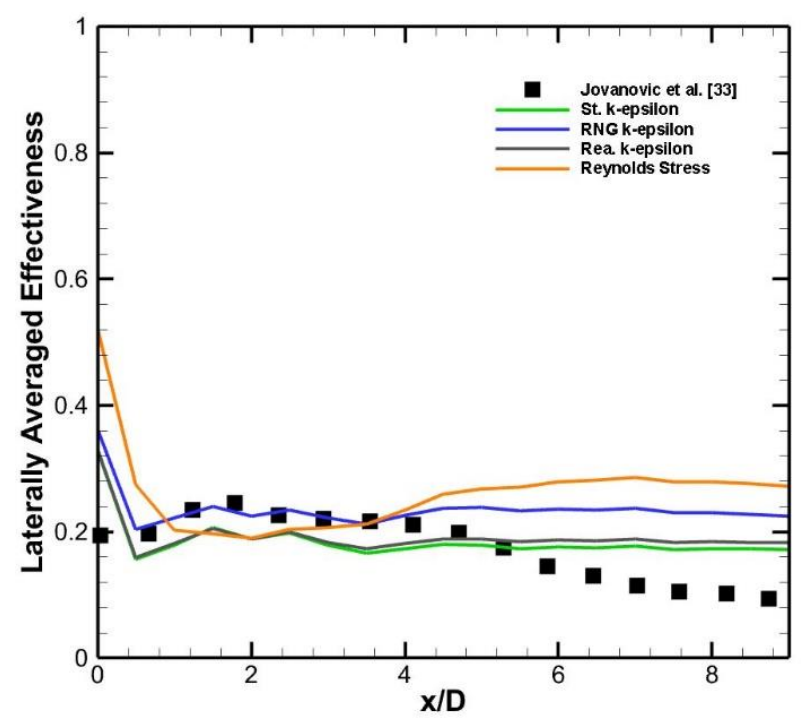

c) k-epsilon, laterally averaged effectiveness

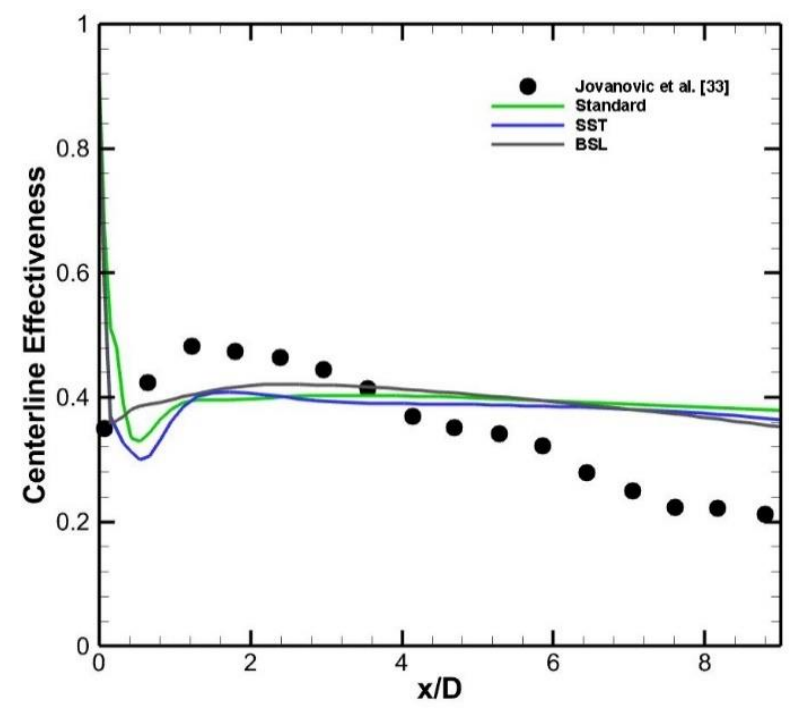

b) k-omega, centerline effectiveness

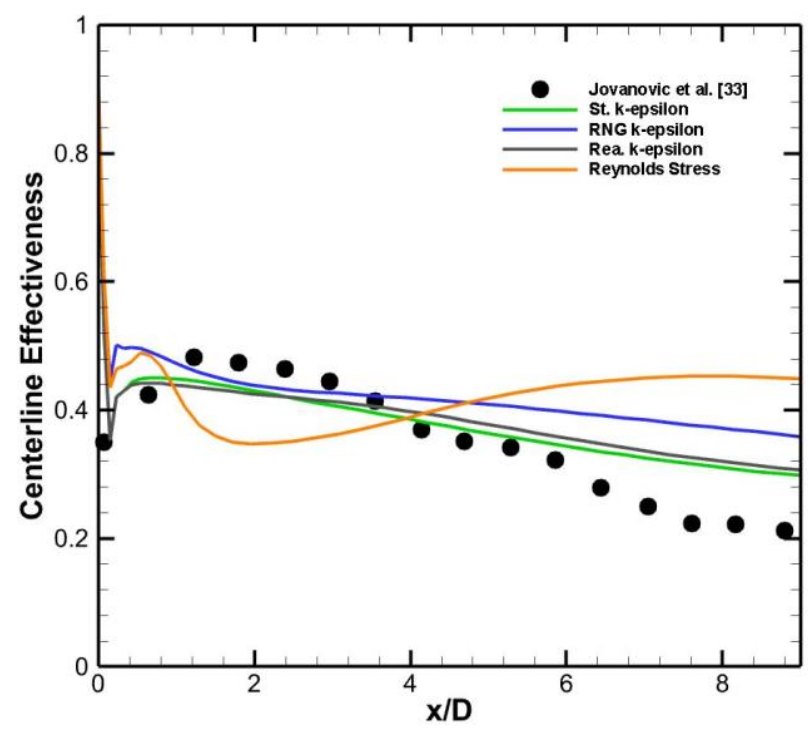

d) k-epsilon, centerline effectiveness

Figure 37. Film Cooling Effectiveness at a Blowing Ratio of 0.90 and Density Ratio of 1 at $\mathrm{Y}+$ of 1 for the Perfect Case 


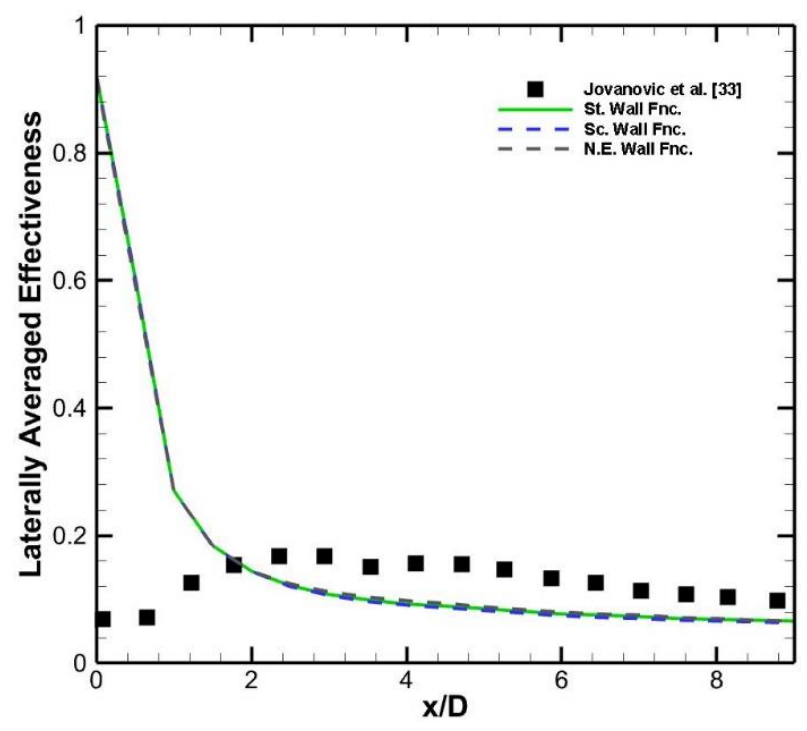

a) St. k-epsilon

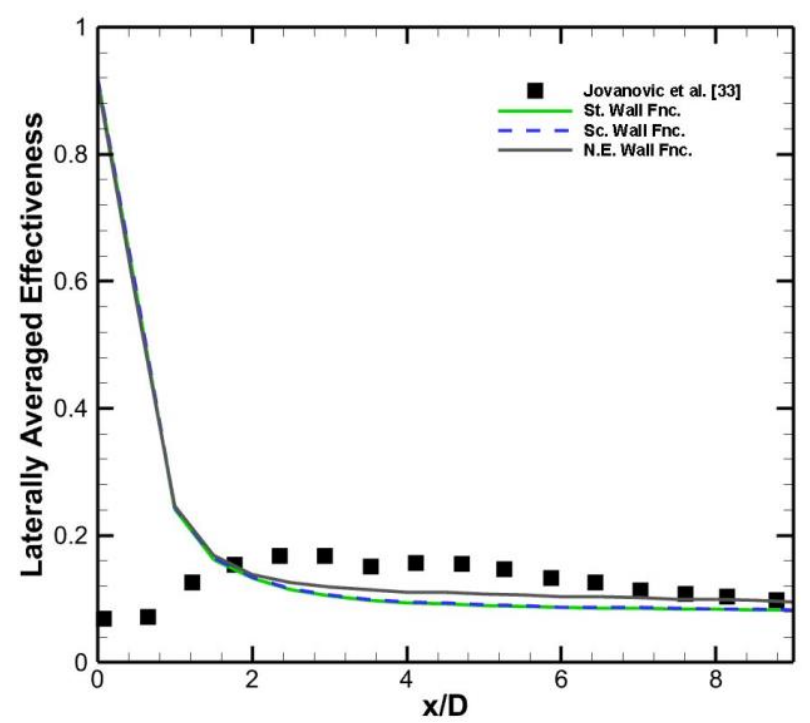

c) Realizable k-epsilon

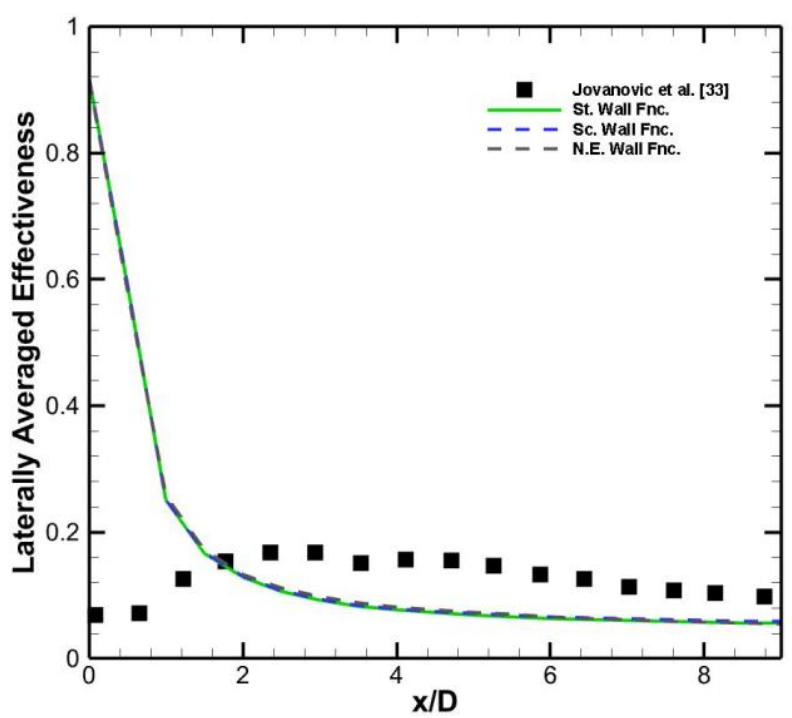

b) RNG k-epsilon

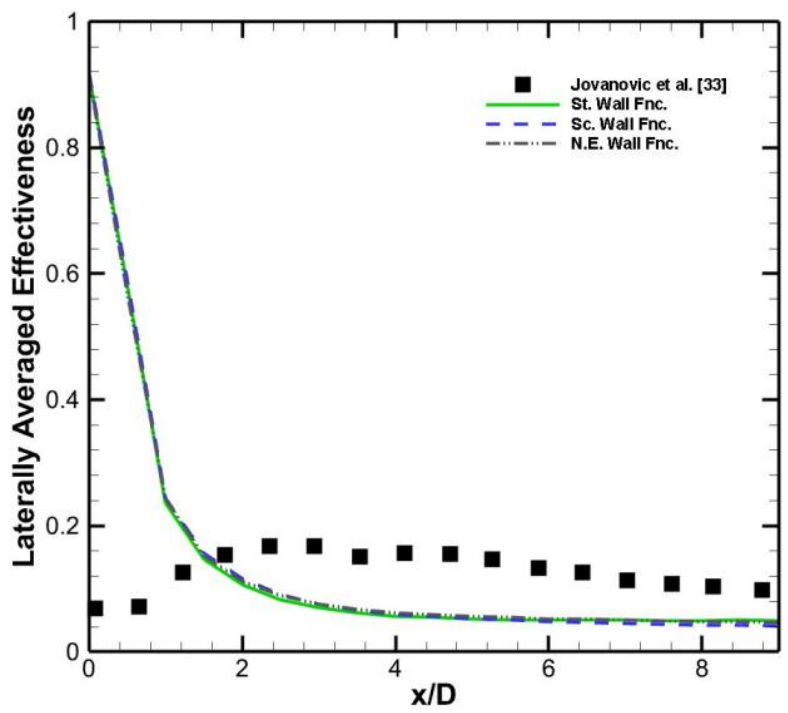

d) Reynolds Stress

Figure 38. Laterally Averaged Film Cooling Effectiveness at a Blowing Ratio of 1.25 and Density Ratio of 1 at $\mathrm{Y}+$ of 30 for the Perfect Case 


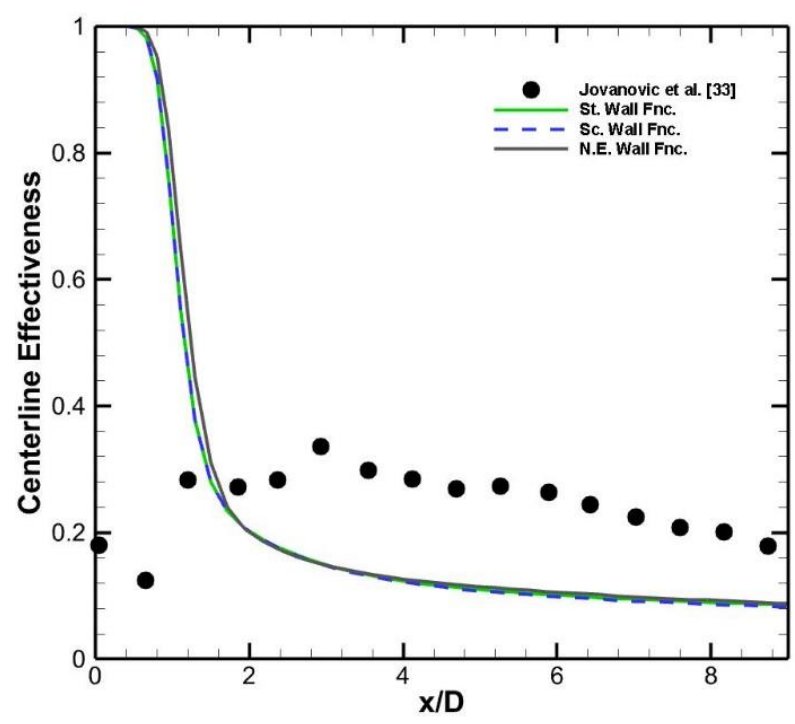

a) St. k-epsilon

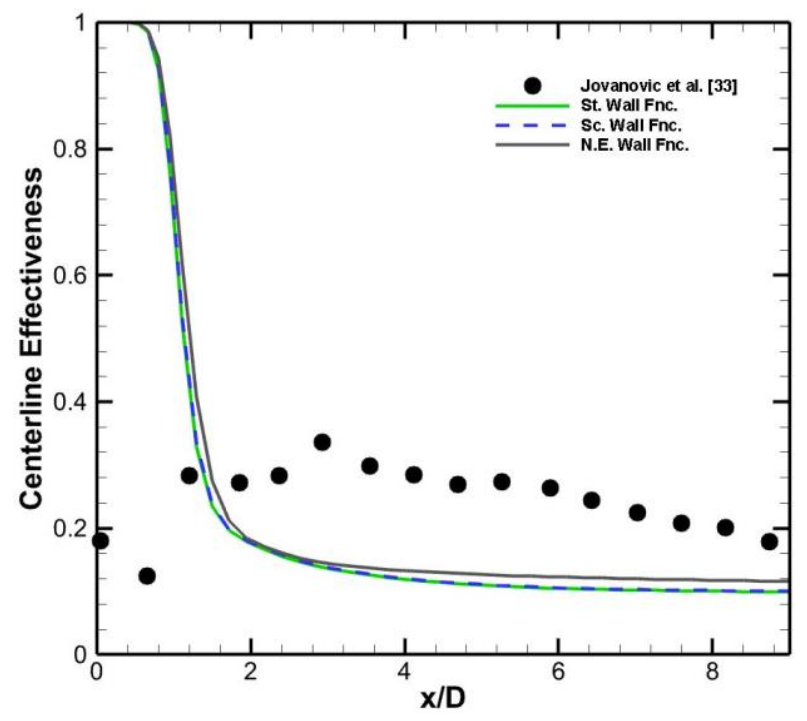

c) Realizable k-epsilon

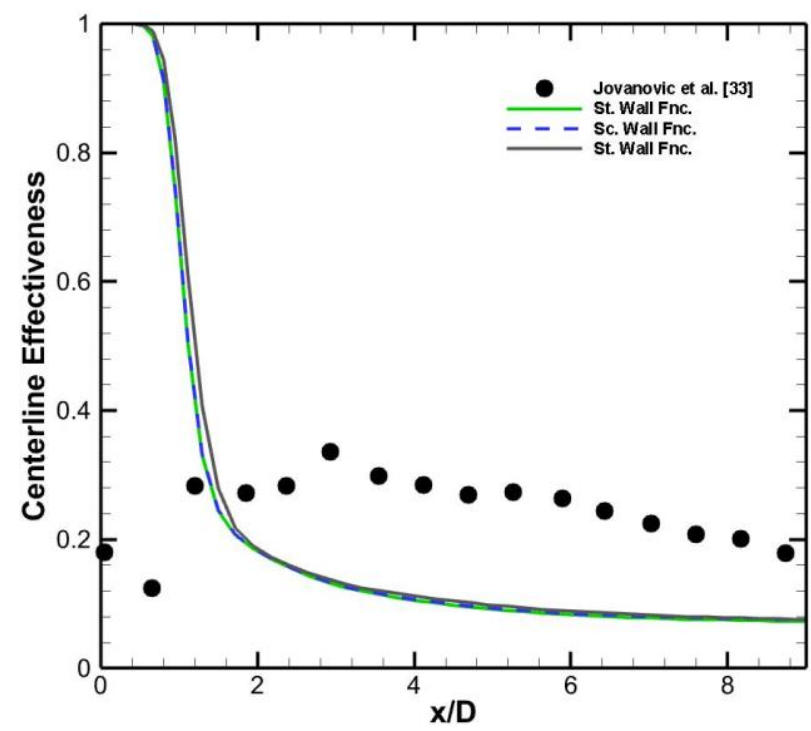

b) RNG k-epsilon

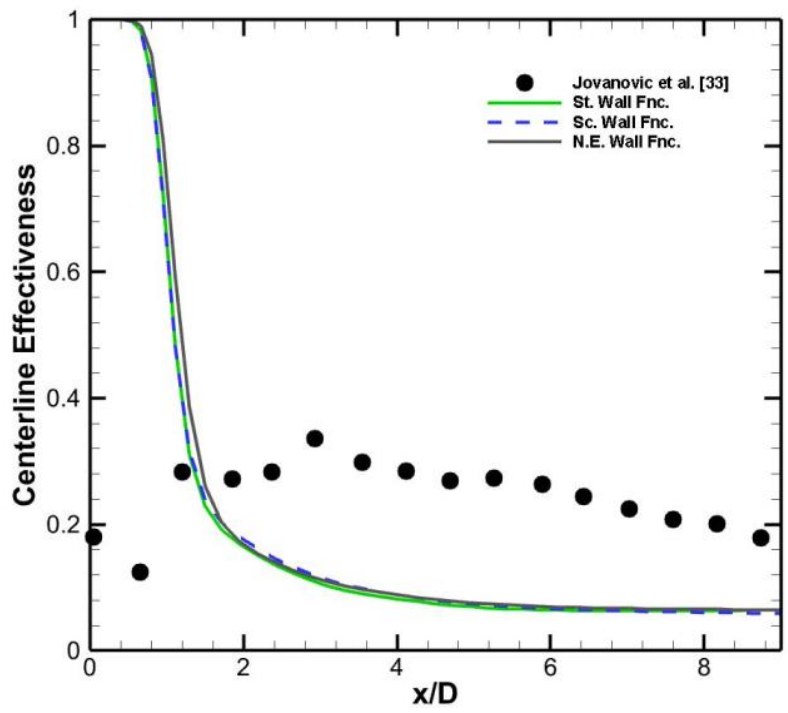

d) Reynolds Stress

Figure 39. Centerline Film Cooling Effectiveness at a Blowing Ratio of 1.25 and Density Ratio of 1 at $\mathrm{Y}+$ of 30 for the Perfect Case 


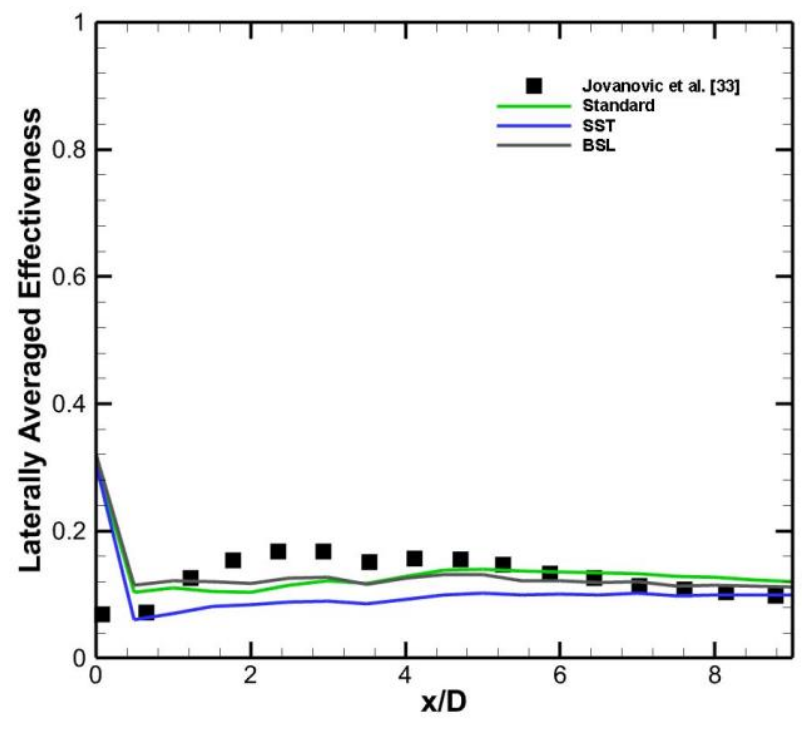

a) k-omega, laterally averaged effectiveness

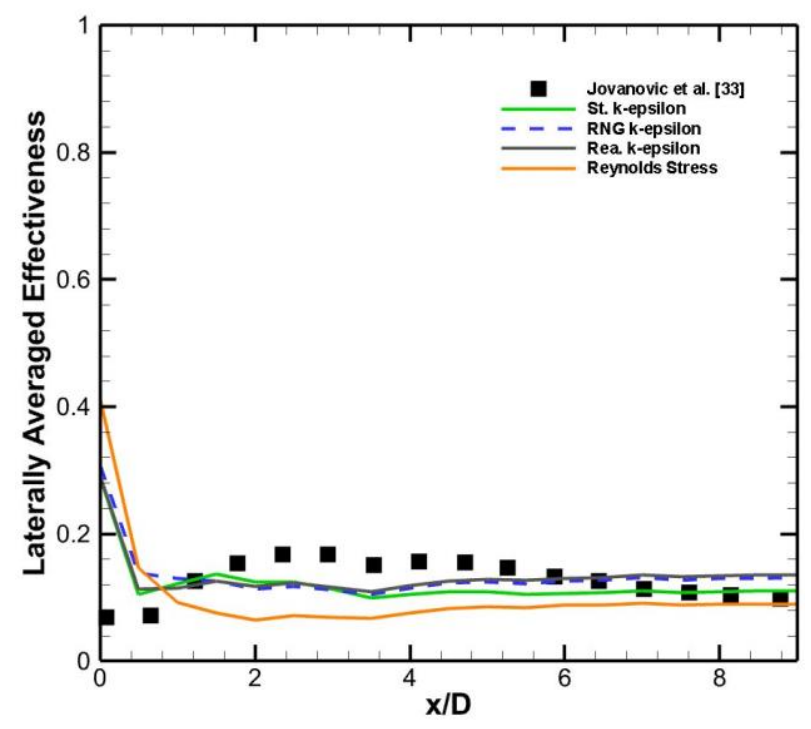

c) k-epsilon, laterally averaged effectiveness

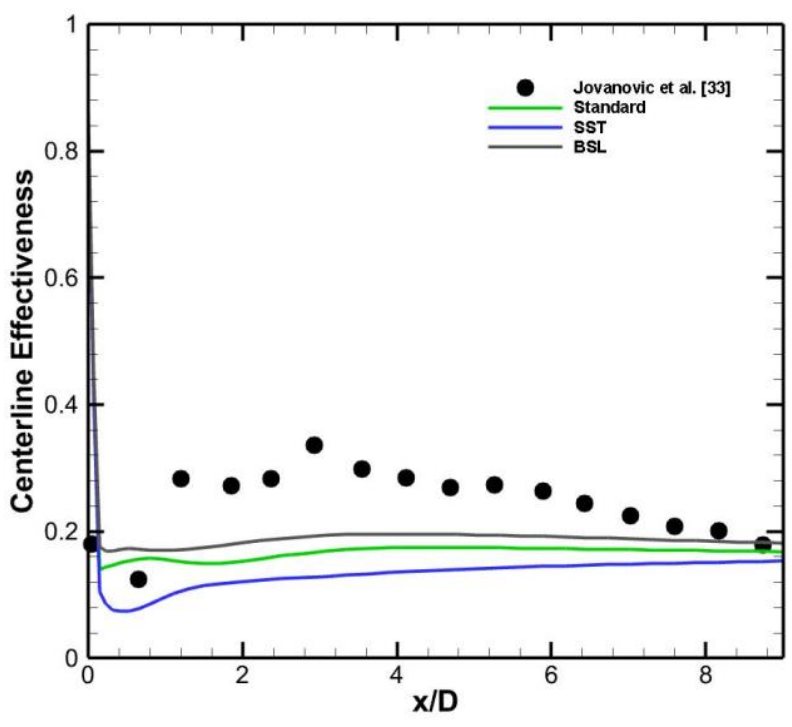

b) k-omega, centerline effectiveness

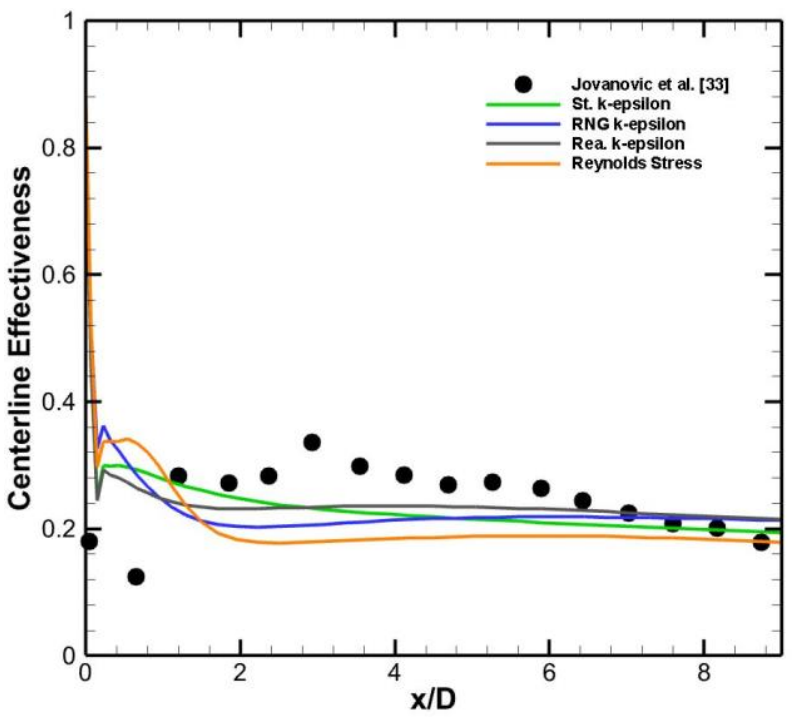

d) k-epsilon, centerline effectiveness

Figure 40. Film Cooling Effectiveness at a Blowing Ratio of 1.25 and Density Ratio of 1 at $Y+$ of 1 for the Perfect Case 


\section{Imperfect Case}

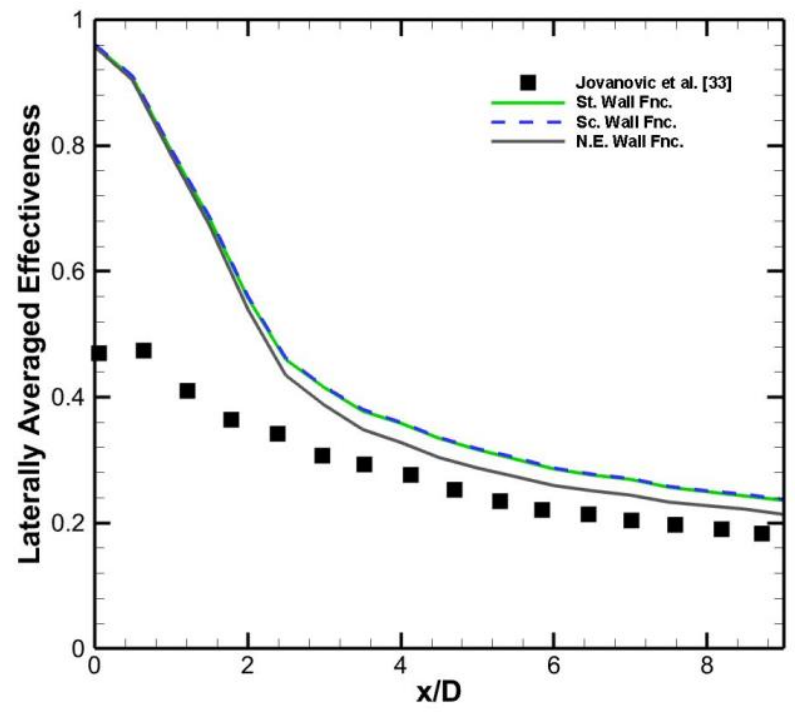

a) St. k-epsilon

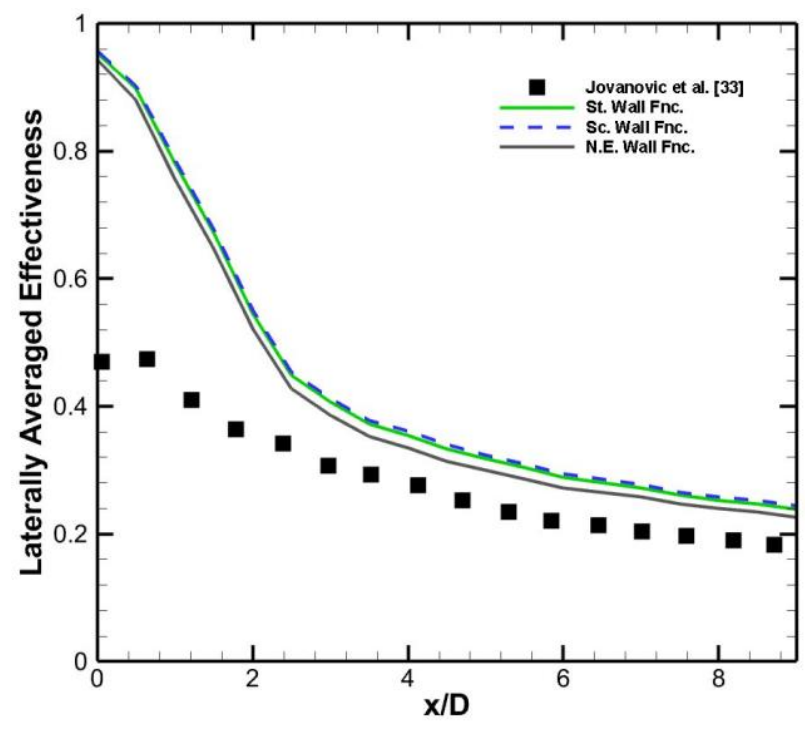

c) Realizable k-epsilon

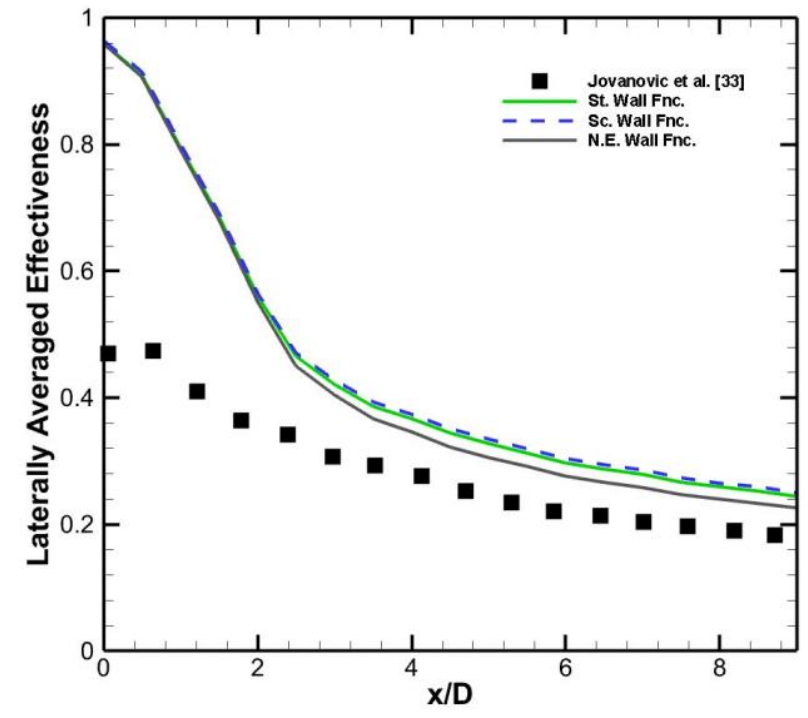

b) RNG k-epsilon

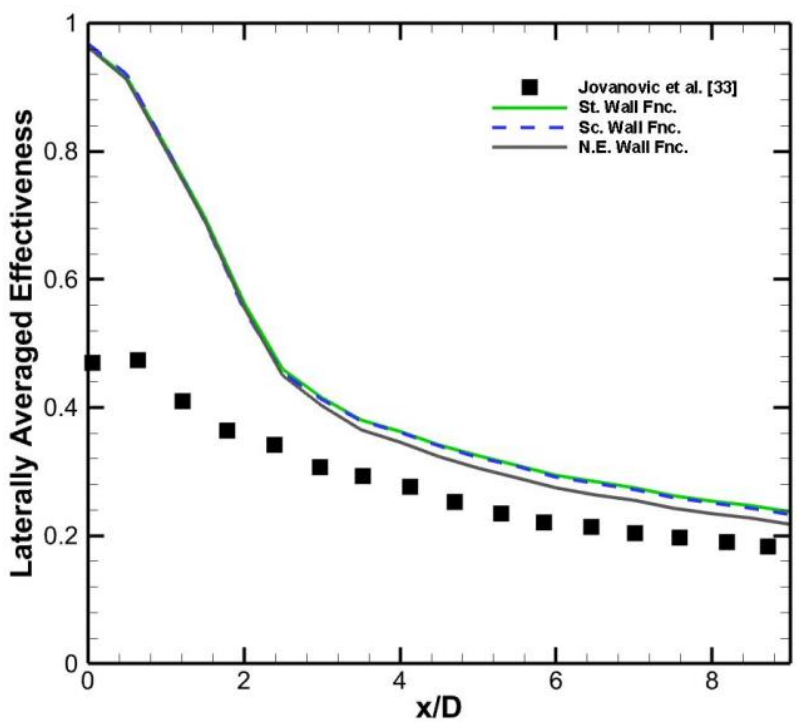

d) Reynolds Stress

Figure 41. Laterally Averaged Film Cooling Effectiveness at a Blowing Ratio of 0.45 and Density Ratio of 1 at $\mathrm{Y}+$ of 30 for the Imperfect Case 


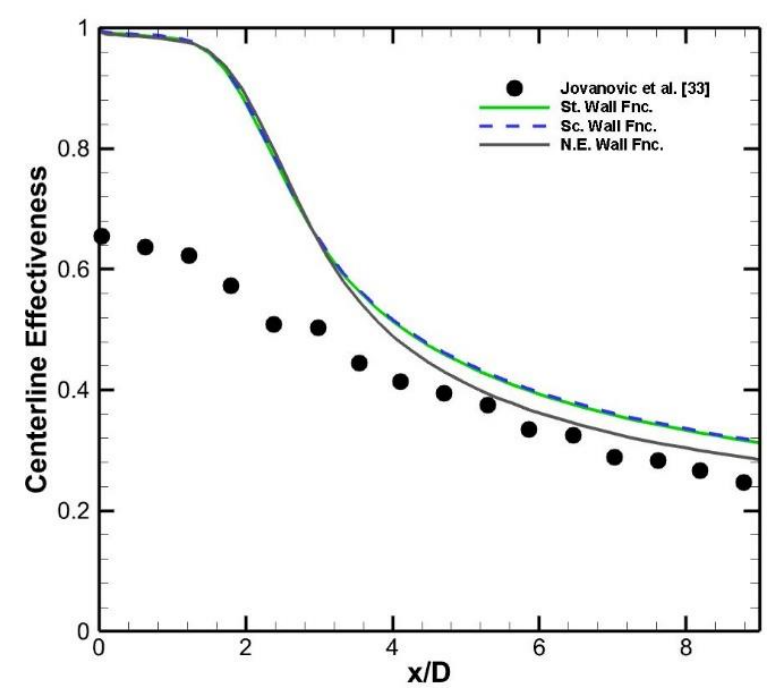

a) St. k-epsilon

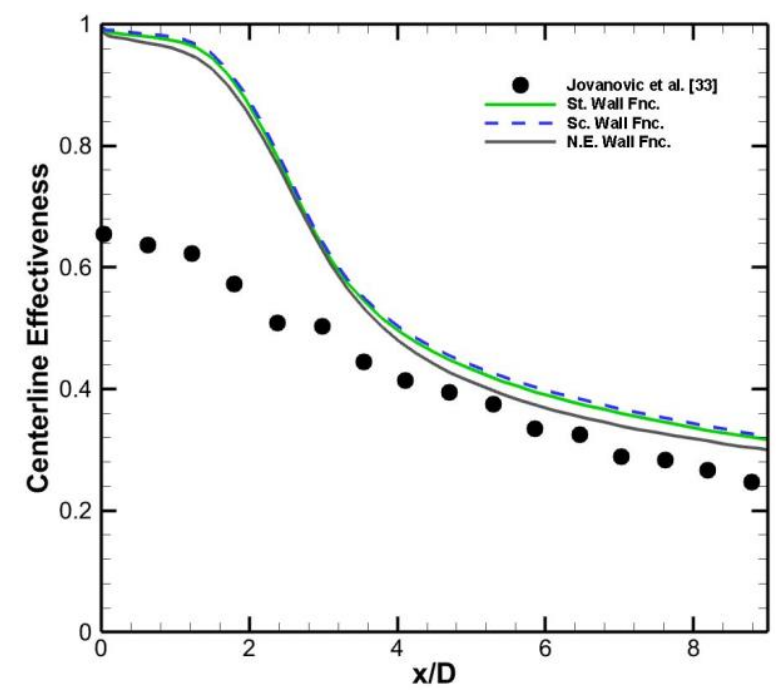

c) Realizable k-epsilon

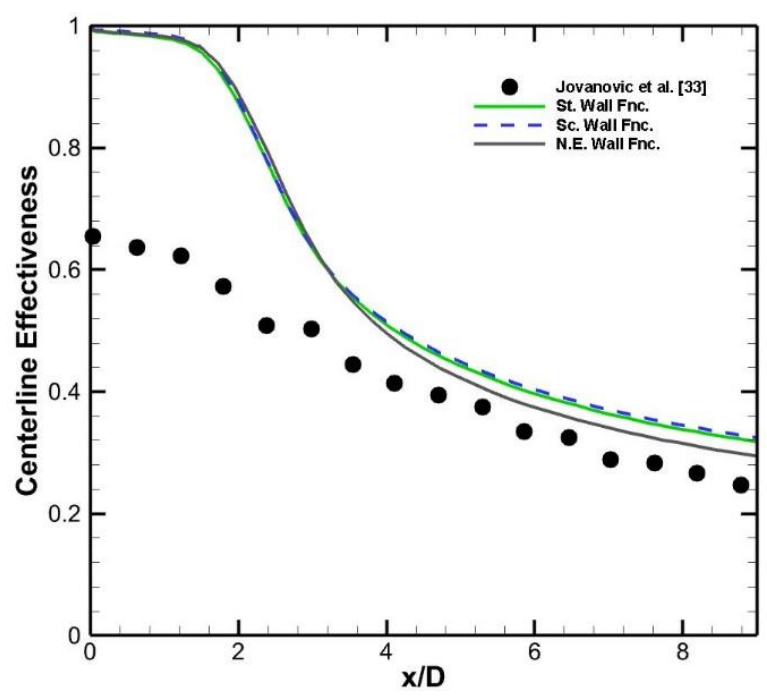

b) RNG k-epsilon

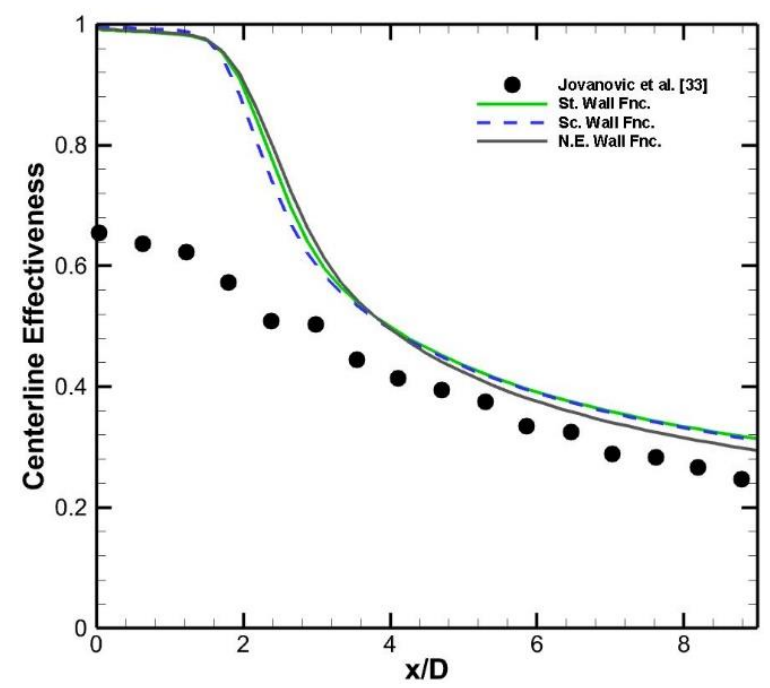

d) Reynolds Stress

Figure 42. Centerline Film Cooling Effectiveness at a Blowing Ratio of 0.45 and Density Ratio of 1 at $\mathrm{Y}+$ of 30 for the Imperfect Case 


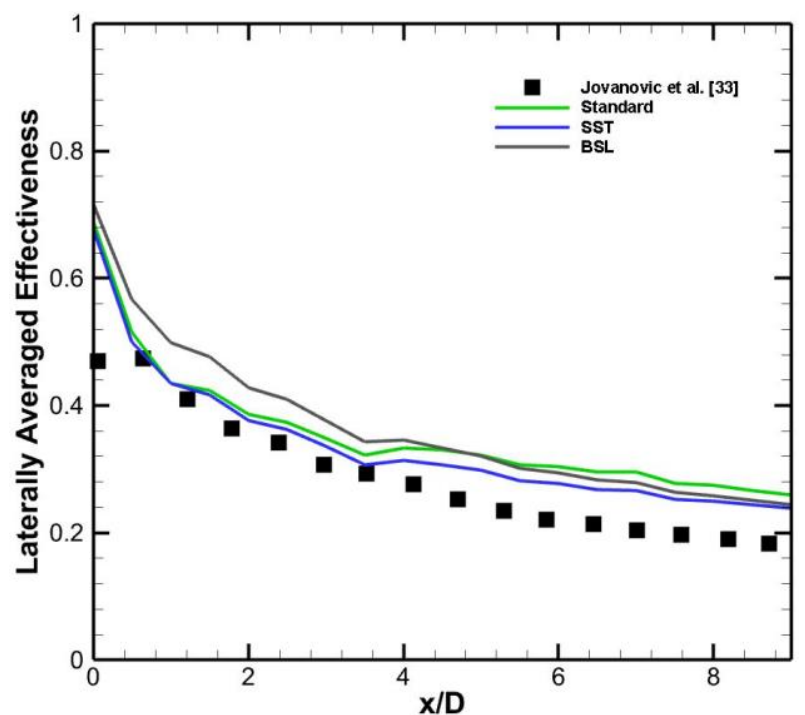

a) k-omega, laterally averaged effectiveness

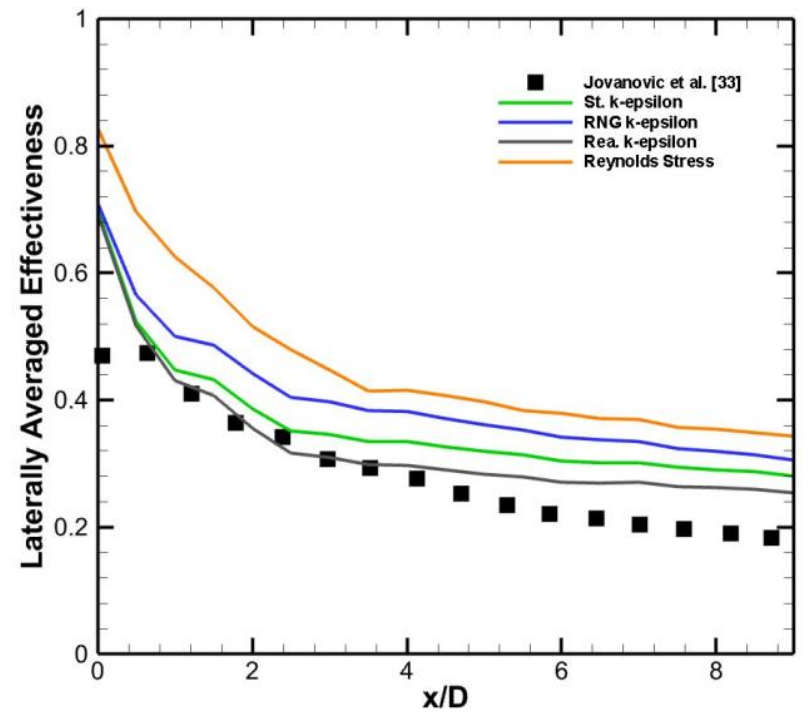

c) k-epsilon, laterally averaged effectiveness

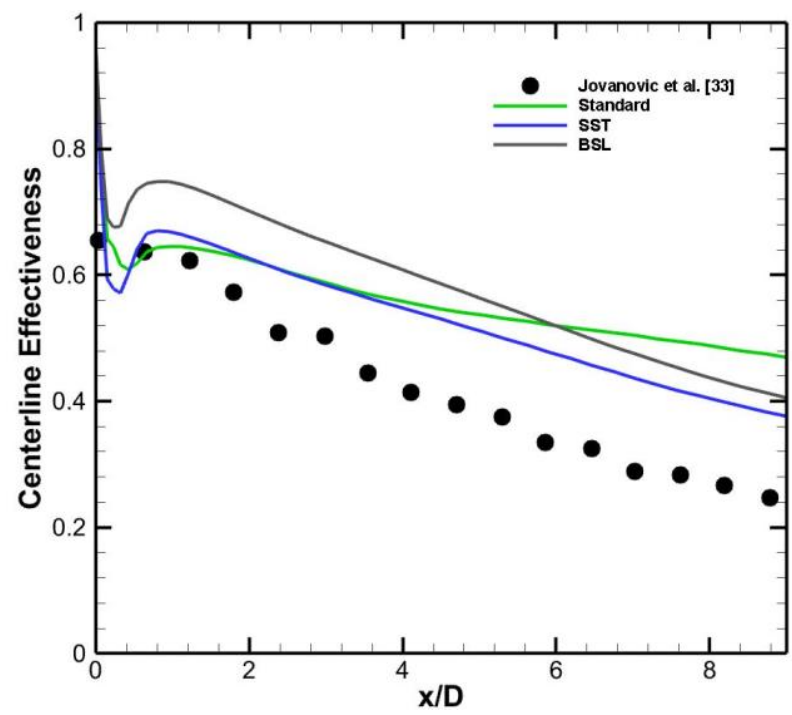

b) k-omega, centerline effectiveness

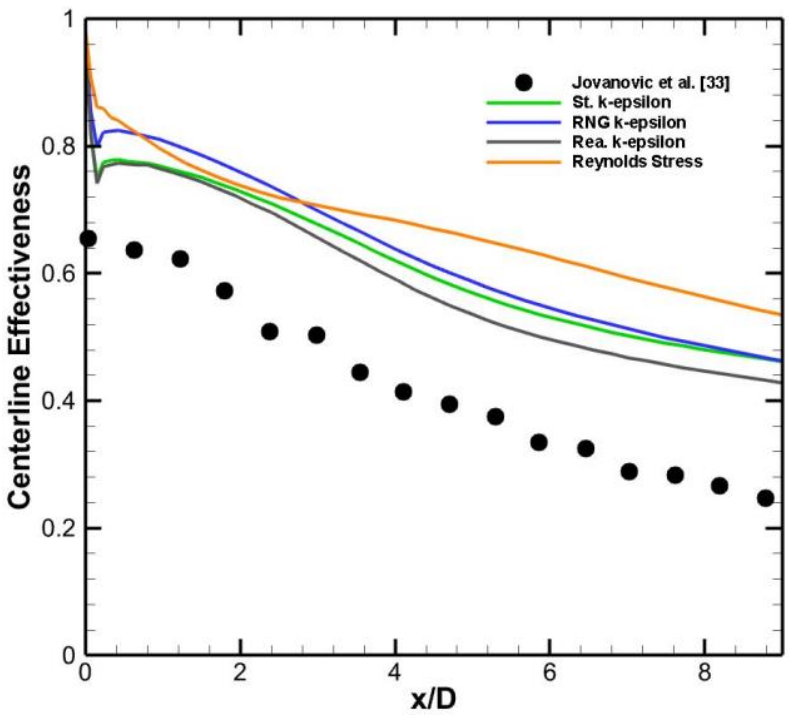

d) k-epsilon, centerline effectiveness

Figure 43. Film Cooling Effectiveness at a Blowing Ratio of 0.45 and Density Ratio of 1 at $Y+$ of 1 for the Imperfect Case 


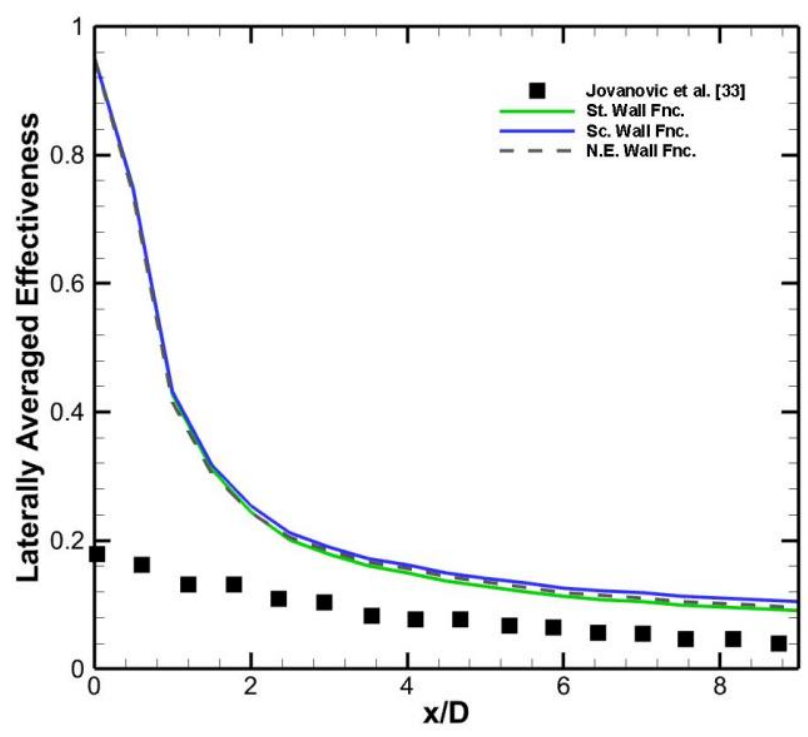

a) St. k-epsilon

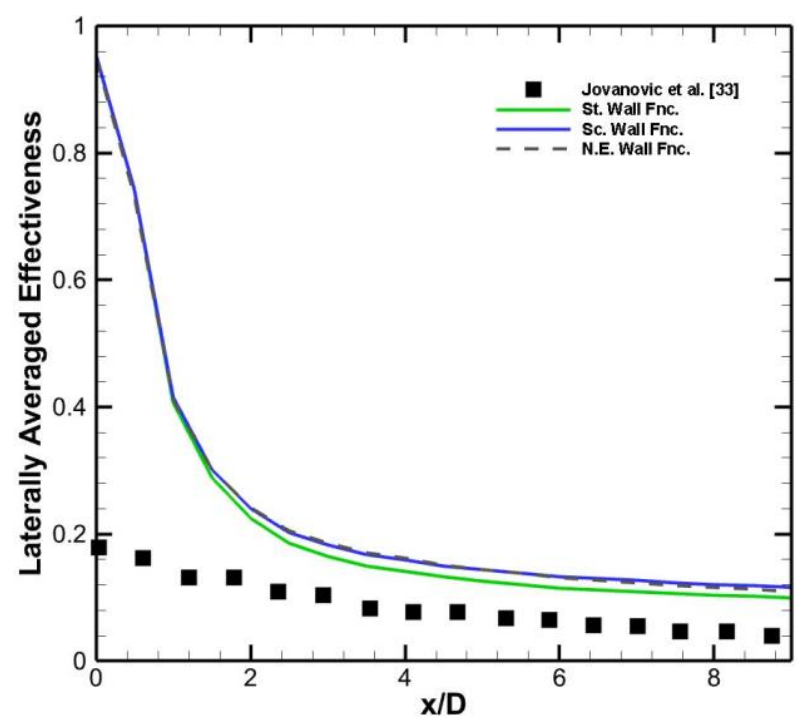

c) Realizable k-epsilon

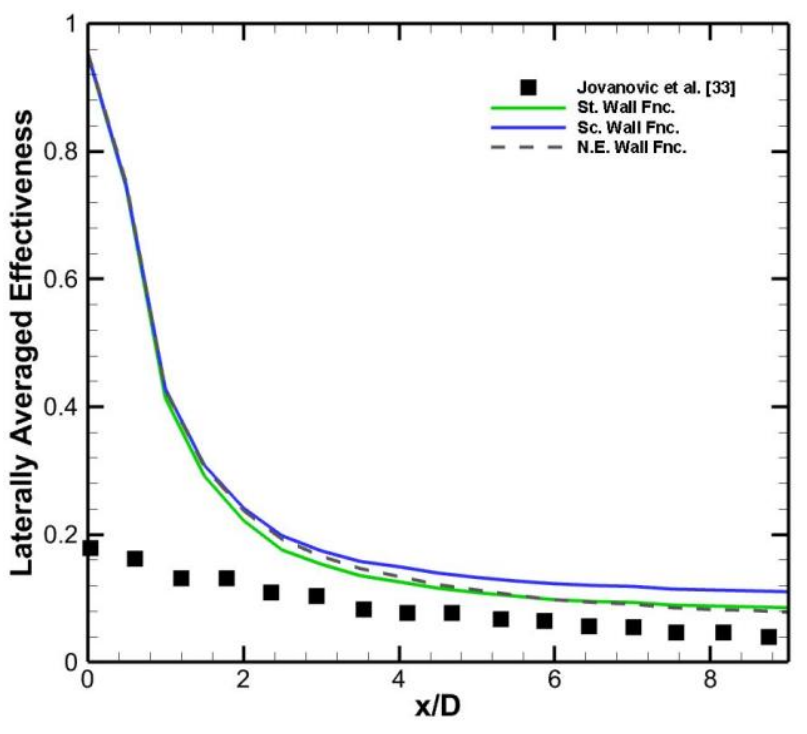

b) RNG k-epsilon

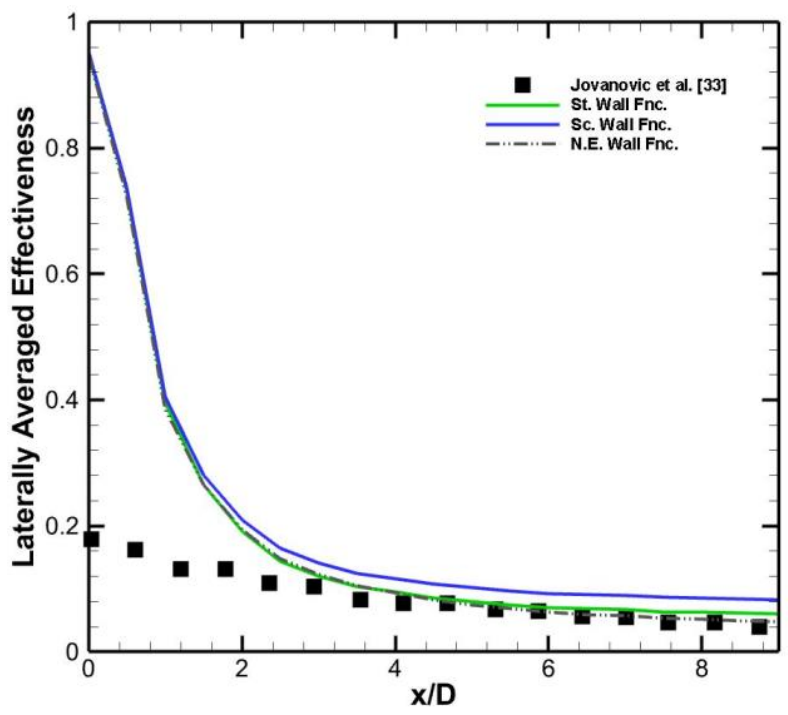

d) Reynolds Stress

Figure 44. Laterally Averaged Film Cooling Effectiveness at a Blowing Ratio of 0.90 and Density Ratio of 1 at $\mathrm{Y}+$ of 30 for the Imperfect Case 


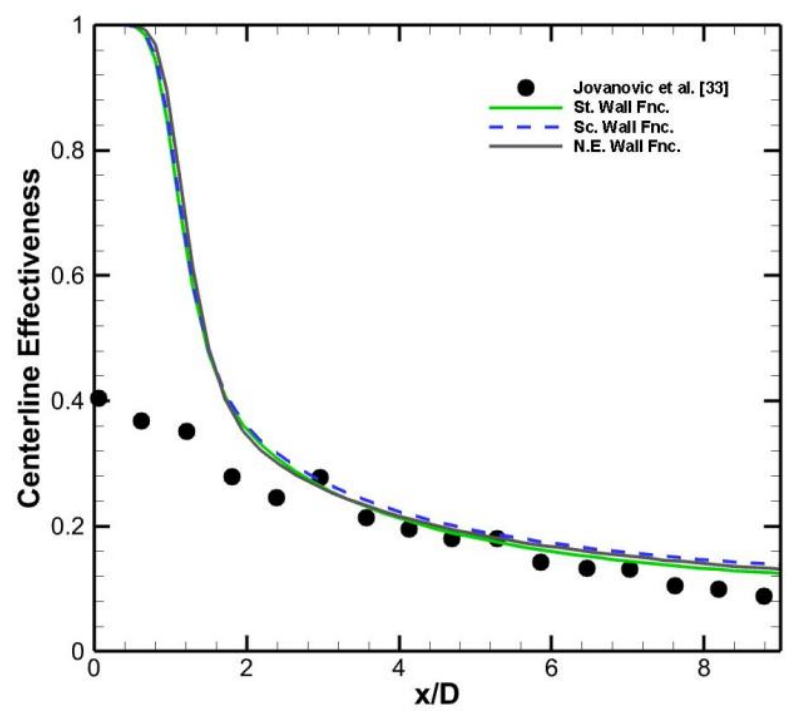

a) St. k-epsilon

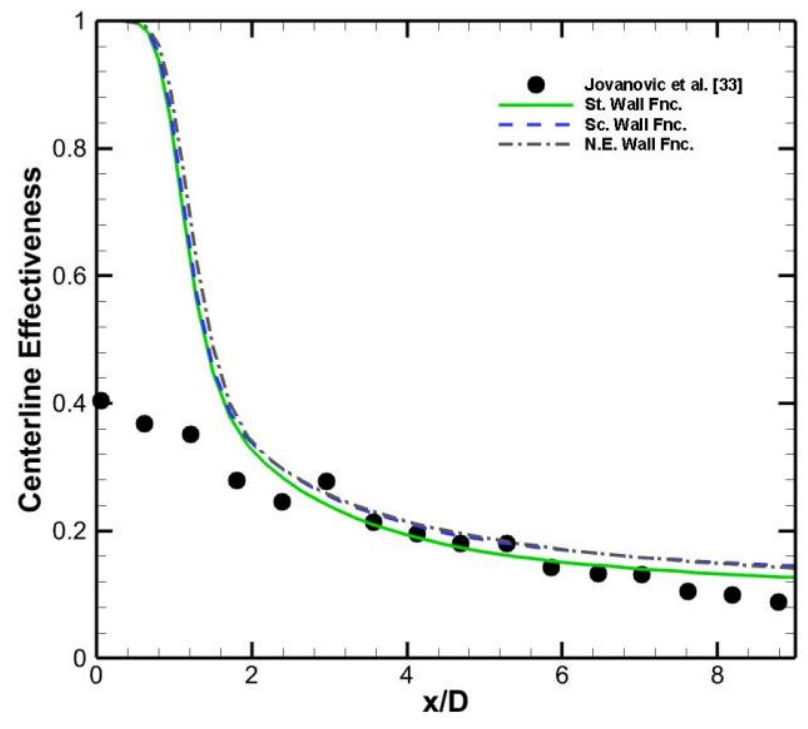

c) Realizable k-epsilon

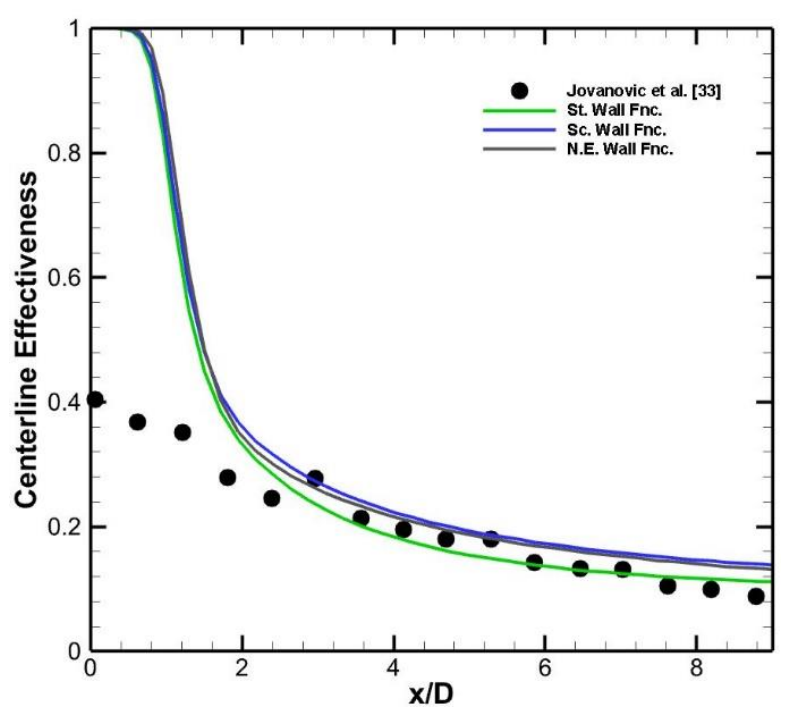

b) RNG k-epsilon

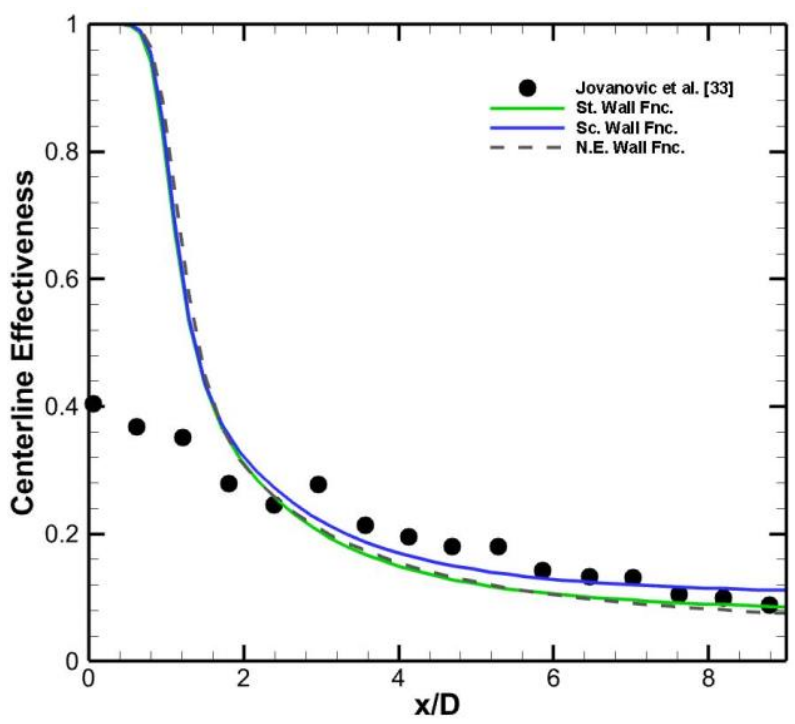

d) Reynolds Stress

Figure 45. Centerline Film Cooling Effectiveness at a Blowing Ratio of 0.90 and Density Ratio of 1 at $\mathrm{Y}+$ of 30 for the Imperfect Case 


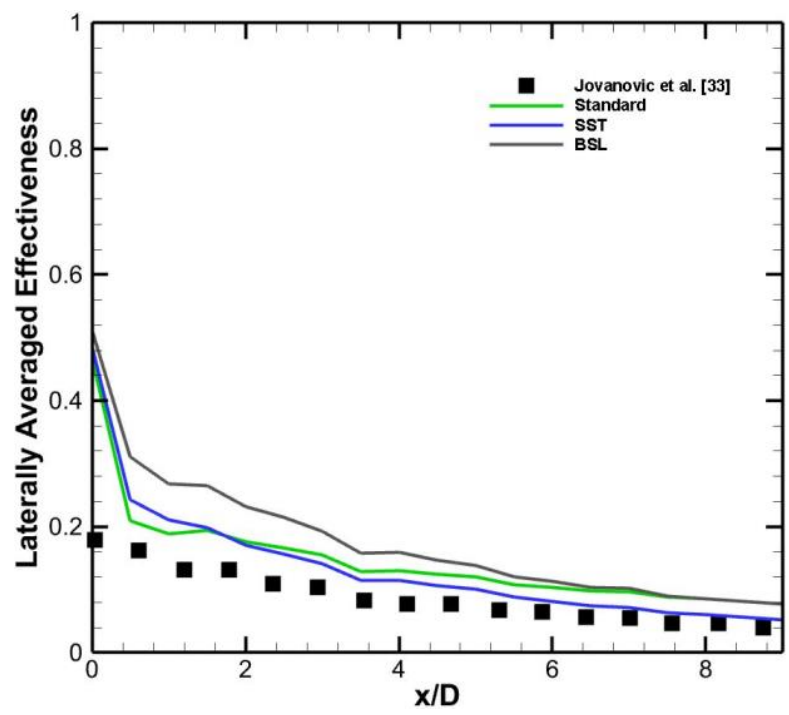

a) k-omega, laterally averaged effectiveness

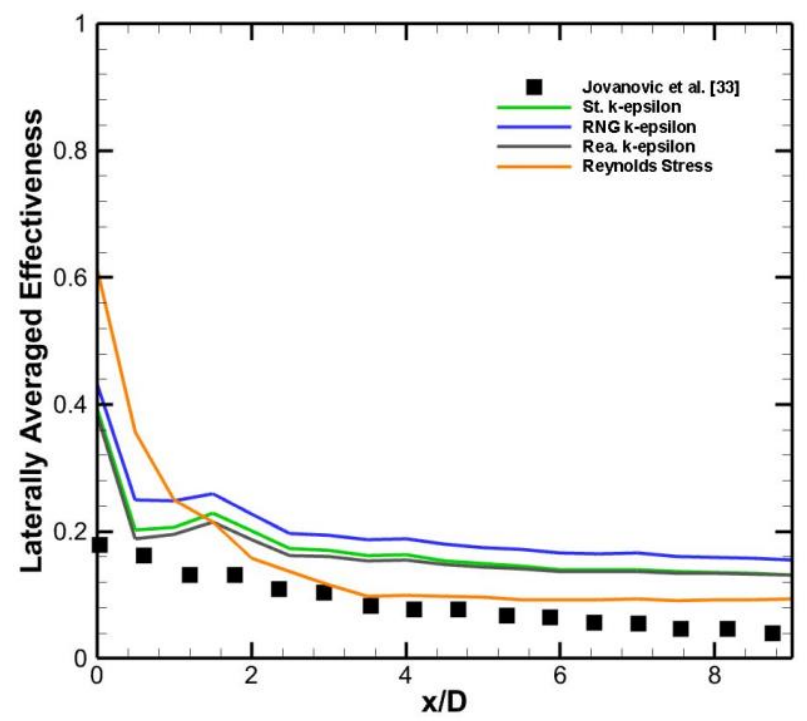

c) k-epsilon, laterally averaged effectiveness

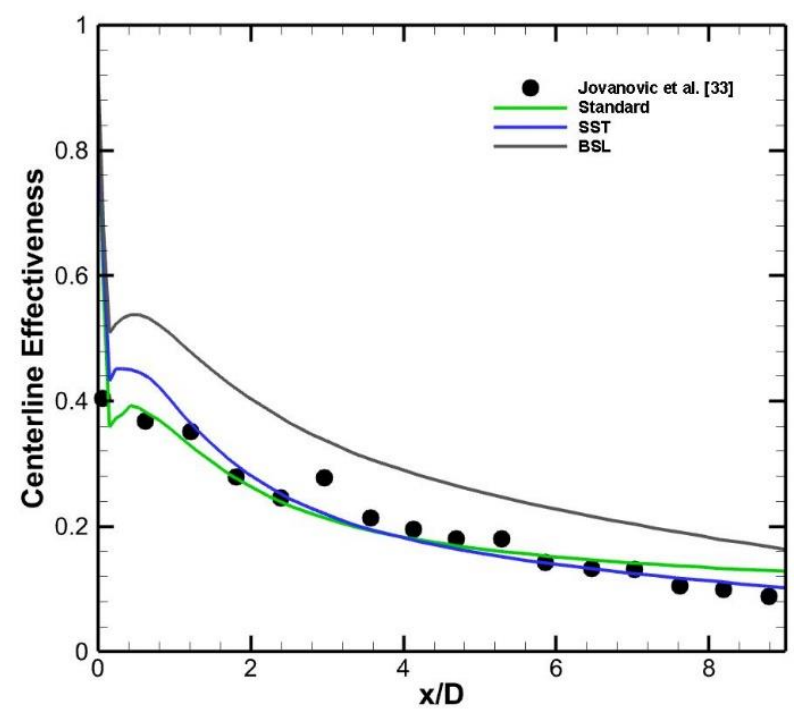

b) k-omega, centerline effectiveness

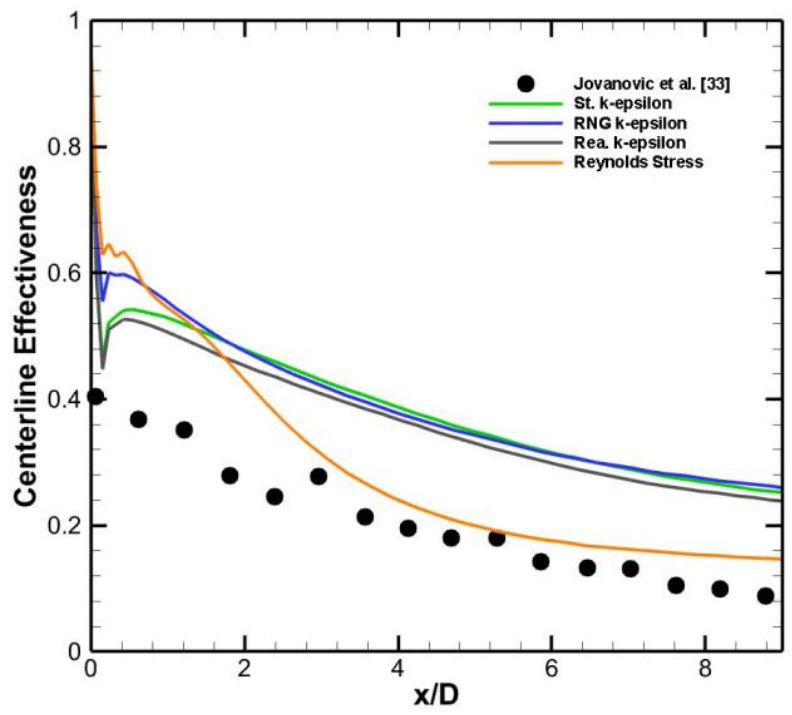

d) k-epsilon, centerline effectiveness

Figure 46. Film Cooling Effectiveness at a Blowing Ratio of 0.90 and Density Ratio of 1 at $Y+$ of 1 for the Imperfect Case 


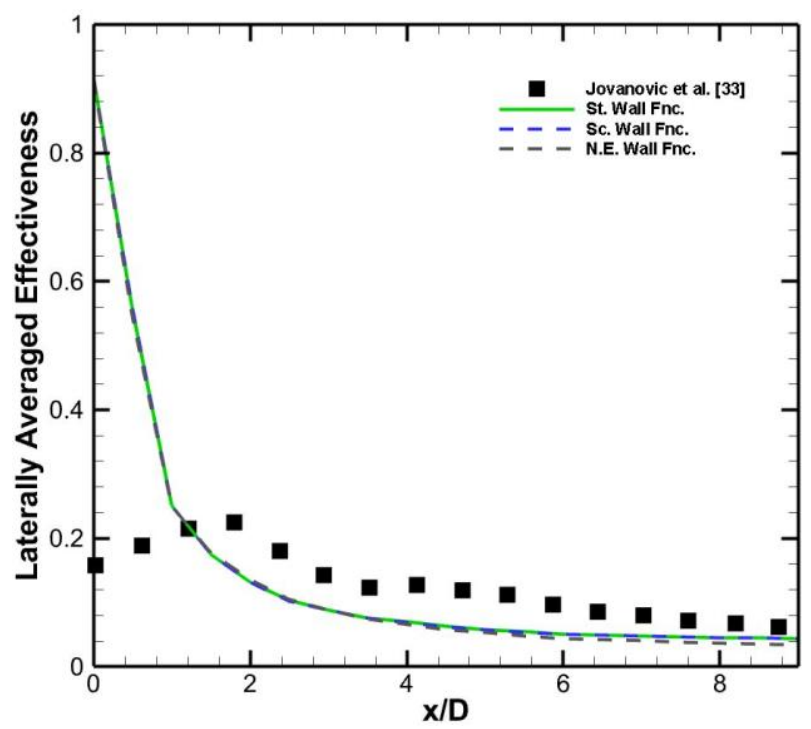

a) St. k-epsilon

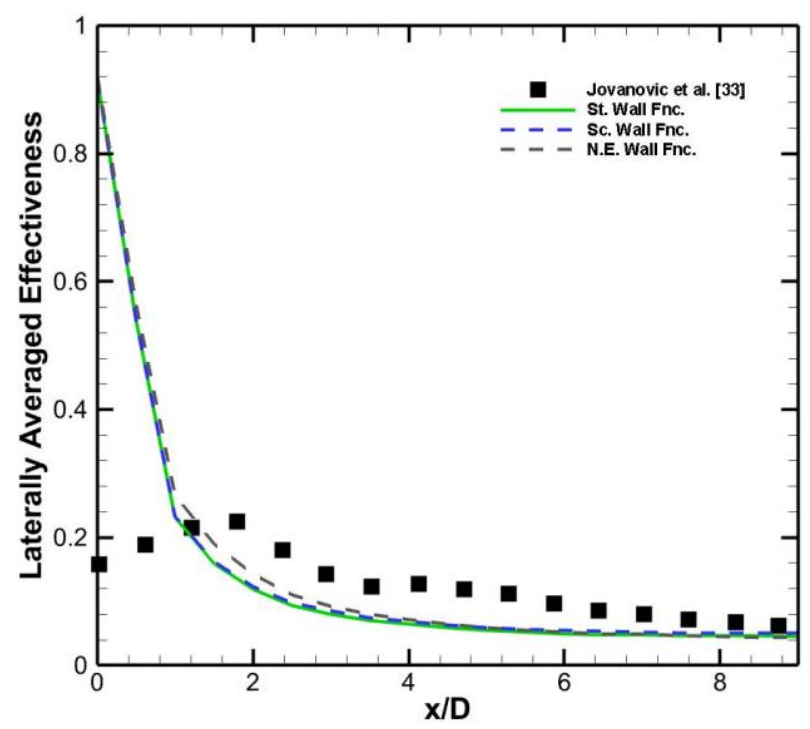

c) Realizable k-epsilon

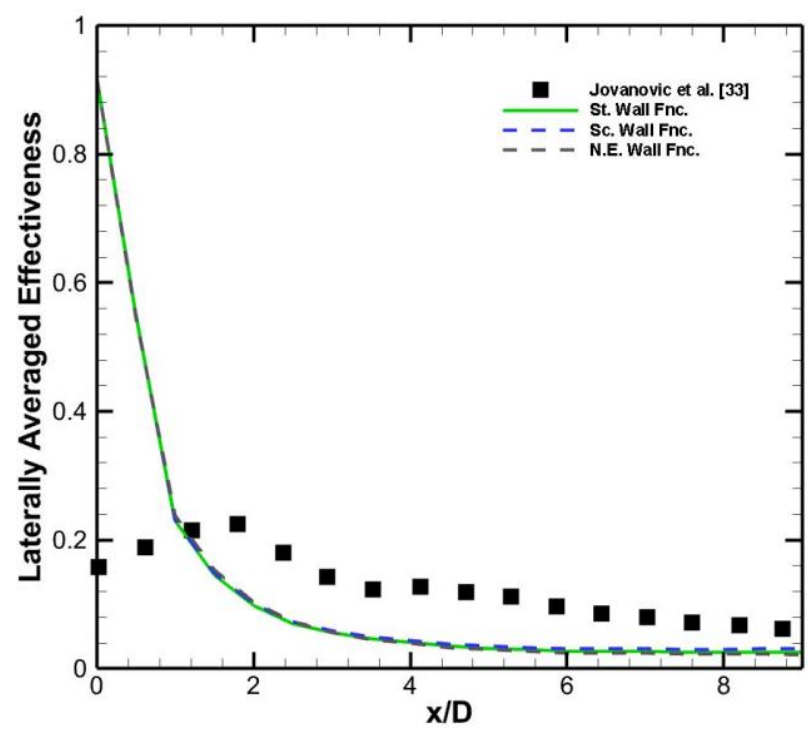

b) RNG k-epsilon

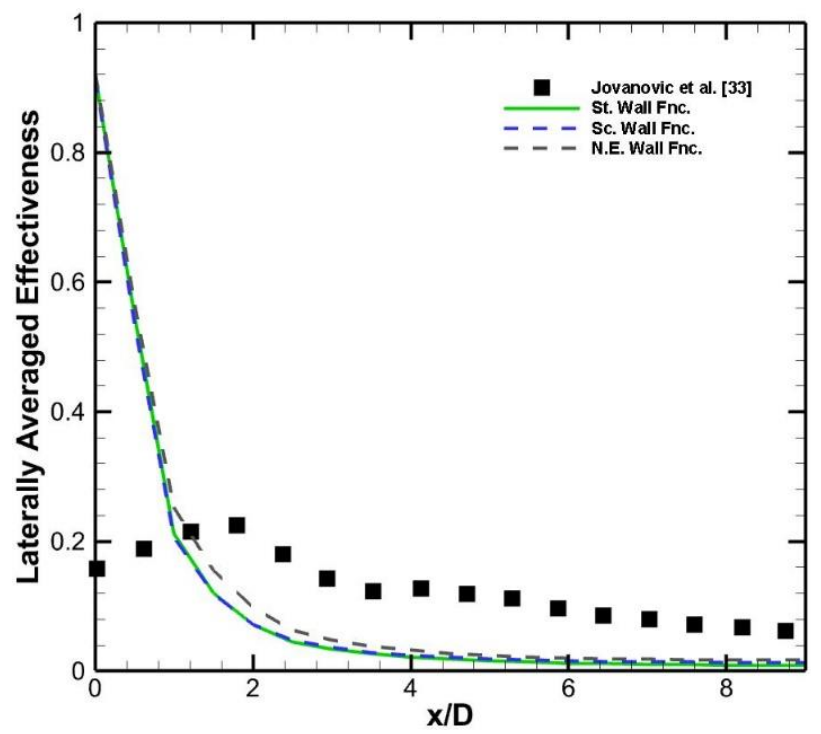

d) Reynolds Stress

Figure 47. Laterally Averaged Film Cooling Effectiveness at a Blowing Ratio of 1.25 and Density Ratio of 1 at $\mathrm{Y}+$ of 30 for the Imperfect Case 


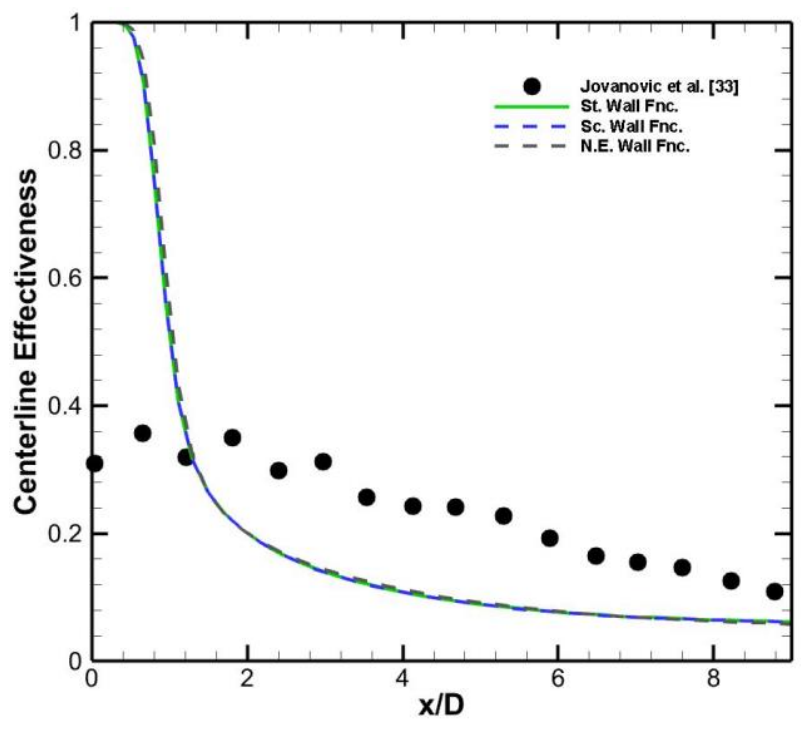

a) St. k-epsilon

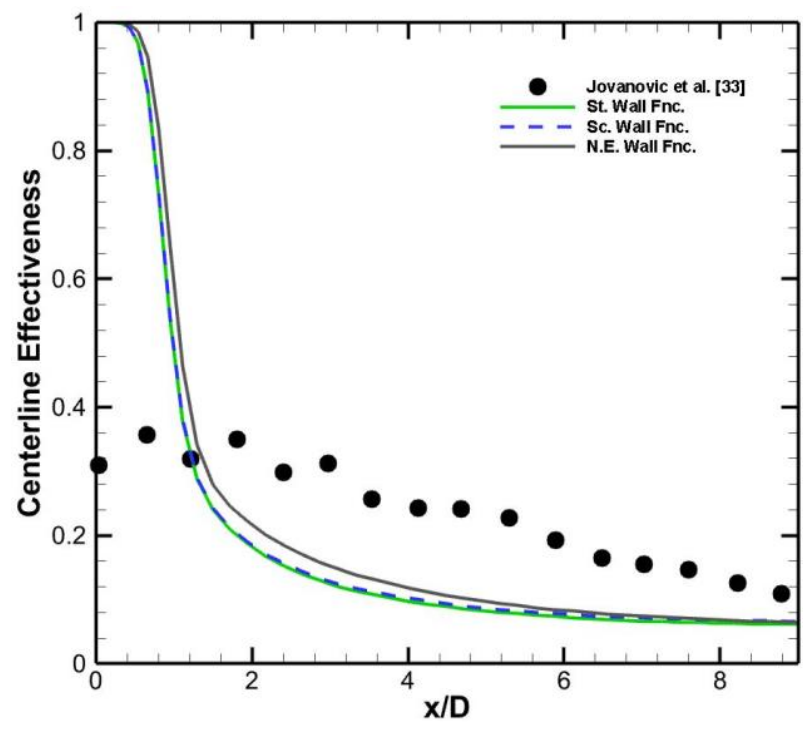

c) Realizable k-epsilon

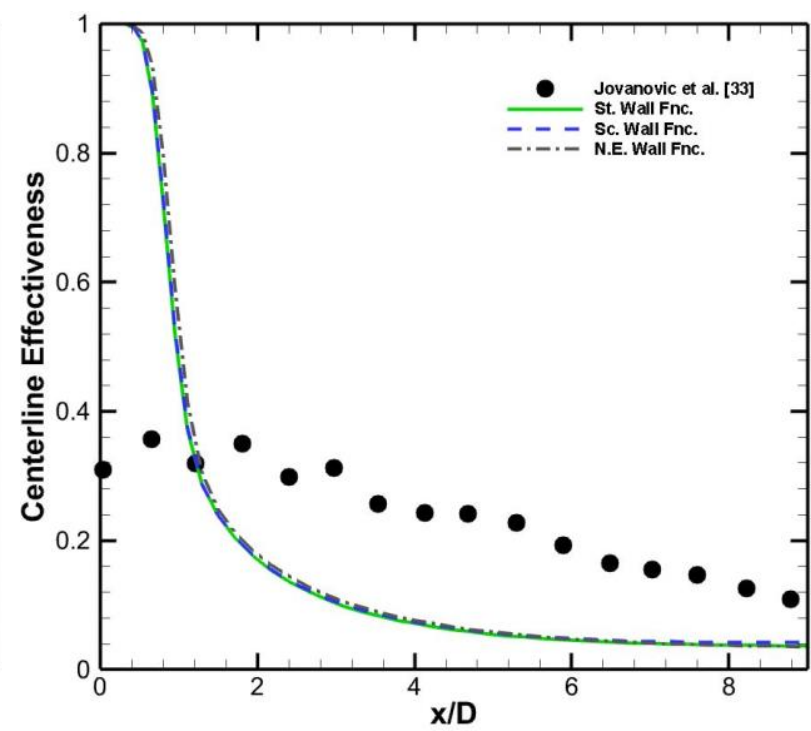

b) RNG k-epsilon

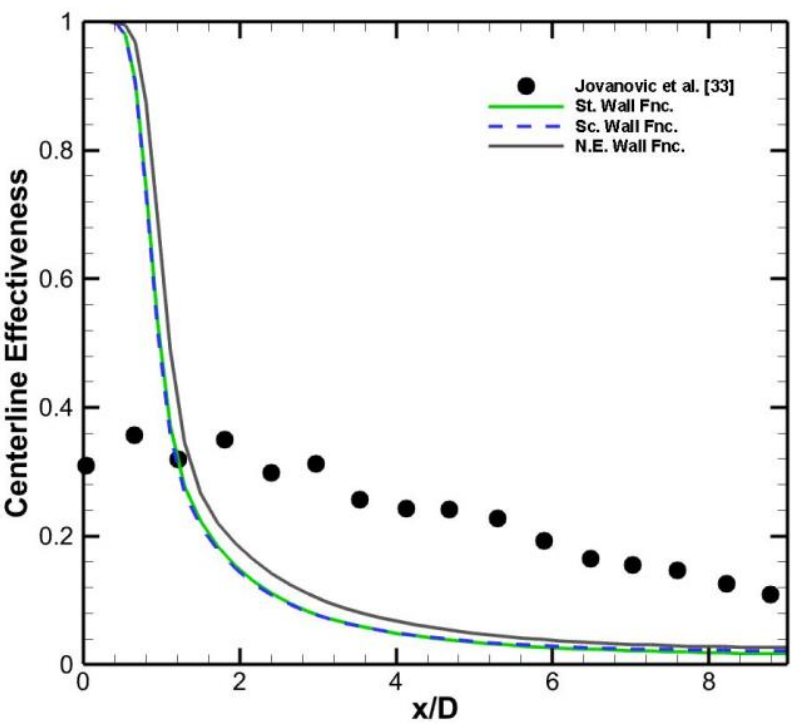

d) Reynolds Stress

Figure 48. Centerline Film Cooling Effectiveness at a Blowing Ratio of 1.25 and Density Ratio of 1 at $\mathrm{Y}+$ of 30 for the Imperfect Case 


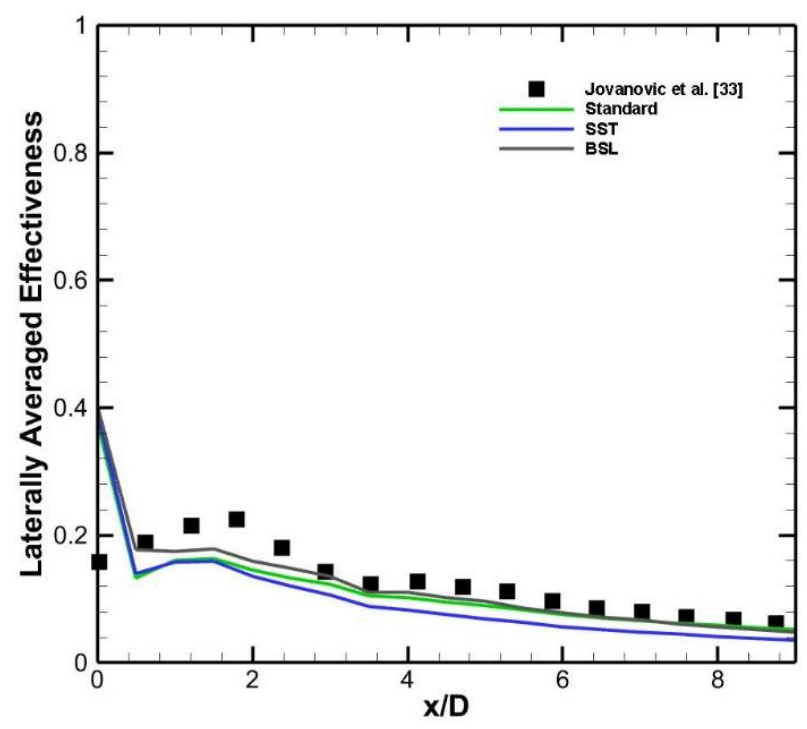

a) k-omega, laterally averaged effectiveness

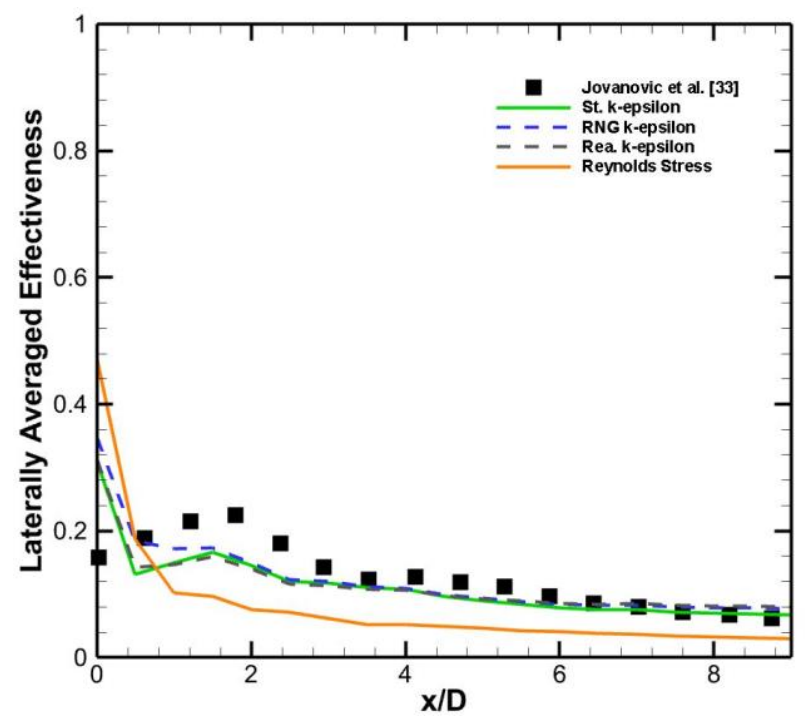

c) k-epsilon, laterally averaged effectiveness

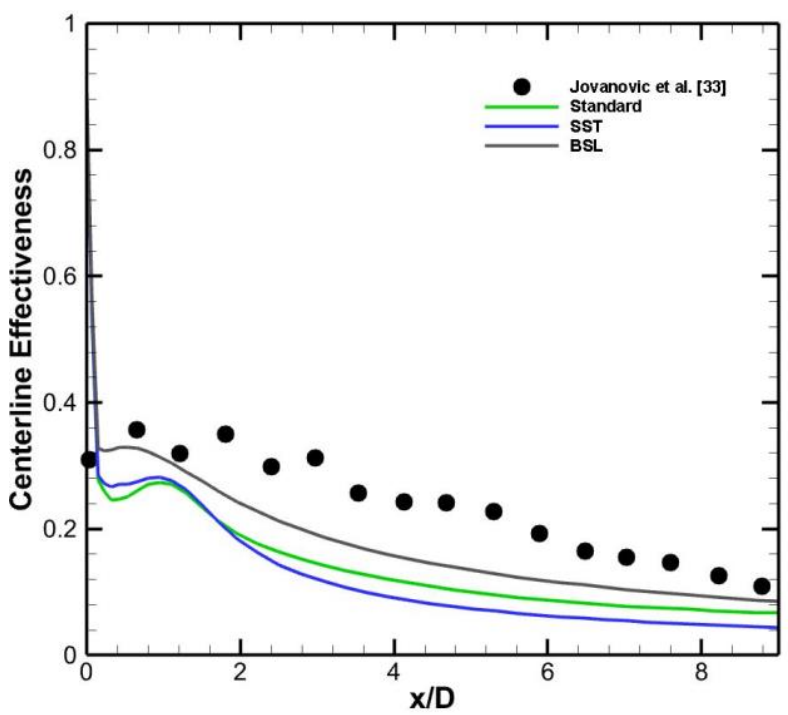

b) k-omega, centerline effectiveness

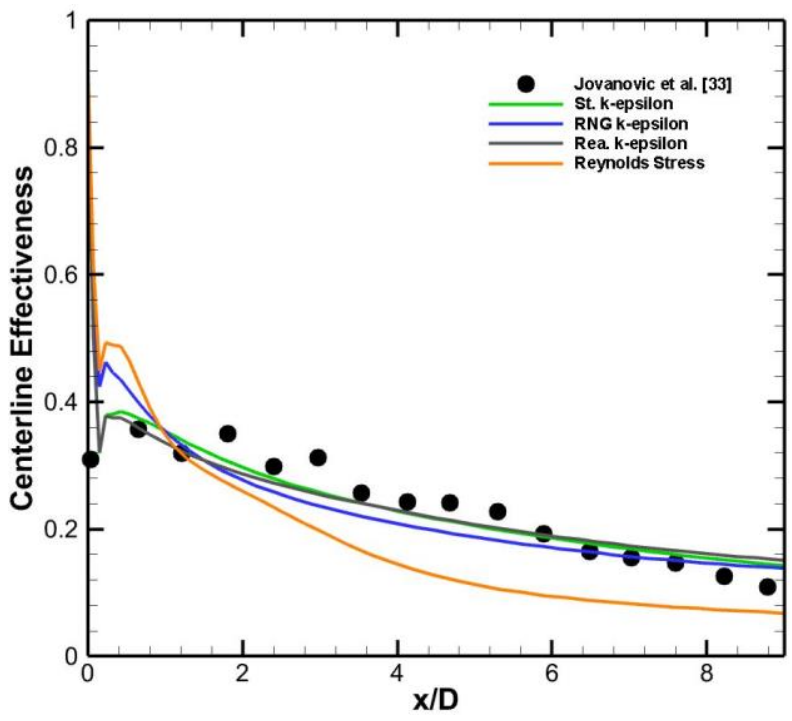

d) k-epsilon, centerline effectiveness

Figure 49. Film Cooling Effectiveness at a Blowing Ratio of 1.25 and Density Ratio of 1 at $\mathrm{Y}+$ of 1 for the Imperfect Case 


\section{REFERENCES}

[1] Mattingly, J.D. (2005). Aircraft Gas Turbine Engine. In Elements of Gas Turbine Propulsion (1 ${ }^{\text {st }}$ ed., pp. 224-236). Chennai, India: Amer Inst of Aeronautics \&.

[2] Moran, M. J., Howard, N.S., Daisie, D.B., Margaret, B.B., Fundamentals of Engineering

Thermodynamics. John Wiley and Sons, 2013.

[3] K.T. Voisey, T.W. Clyne, "Laser drilling of cooling holes through plasma sprayed thermal barrier coatings", Surf. Coat. Technol. 176 (2004) 296-306.

[4] R. Sivakumar, B.L. Mordike, "High temperature coatings for gas turbine blades: a review", Surf. Coat. Technol. 37 (1989) 139-160.

[5] Han, J., Dutta, S., \& Ekkad, S. (2012). Gas Turbine Heat Transfer and Cooling Technology, Second Edition. Boca Raton, FL: CRC Press.

[6] Lakshminarayana, B.: "Fluid Dynamics and Heat Transfer of Turbomachinery". Chapter 7, pp. 597721. 1996. Copyright Wiley-VCH GmbH \& Co. KGaA.

[7] Mohamed Naeem, “Advancement In Laser Drilling For Aerospace Gas Turbines”, Proceedings of the $3^{\text {rd }}$ Pacific International Conference on Application of Lasers and Optics 2008.

[8] C. A. MacNally, J. Folkes, and I. R. Pashby, (2004) "Laser drilling of cooling holes in aeroengines: state of the art and future challenges", Material Science and Technology, Vol. 20, pp. 805-813 [9] Jovanovic, M.B., de Lange, H.C., and van Steenhoven, A.A. (2005), "Influence of hole imperfection on jet cross flow interaction", International Journal of Heat and Fluid Flow, Vol. 27, pp. 42-53 [10] Kulkarni, A.V. (2007) "Electrochemical Discharge Machining Process", Defense Science Journal, Vol. 57, No. 5, pp. 765-770

[11] Ahmed, M.S., (1993) "Modern Applications of EDM and ECM", IEE Manufacturing Technology Update

[12] French, P.W., Hand, D.P., Peters, C., Shannon, G.J., Byrd, P., Steen, W.M., (1998) "Investigation of the Nd:YAG laser percussion drilling process using high speed filming”, Proc Laser Materials Processing Conference ICALEO '98, Vol. 85, Part 1 Section B 1-10

[13] French, P.W., Naeem, M., Watkins, K.G., (2003) "Laser Percussion Drilling of Aerospace Material using a 10kW Peak Power Laser using a $400 \mu \mathrm{m}$ Optical Fibre Delivery System”, 22 ${ }^{\text {nd }}$ International Congress on Applications of Lasers \& Electro-Optics (ICALEO 2003), Paper No. 503, Vol. 55

[14] Van Dijk, M.H.H., De Vlieger, J.E., and Brouwer, J.E., (1989) “Lasers in manufacturing”, Sixth Int. Conf. Birmingham, UK, IFS Publications, 237-247.

[15] Corfe, A.G.: Production Eng., 1983, 62, 13-14. 
[16] Pan, C., Zhang, J., Huang, K., (2014), "Numerical Investigation of Partial Blockage Effect on Film Cooling Effectiveness", Mathematical Problems in Engineering, Vol. 2014, Article ID 167193

[17] Zhou, J., Zhang, J., (2016), "Effects of Partial Blockage Inside Film Holes on Overall Cooling Effectiveness of an Integrated Impingement-Fin-Film Cooling Configuration on Blade Pressure Side", Acta Aeronotica et Astronotica Sinica, Vol. 37, No.9, 2729-2738

[18] Huang, K., Zhang, J., Tan, X., Shan, Y., (2018), “Experimental Study on Film Cooling Performance of Imperfect Holes", Chinese Journal of Aeronautics

[19] Bunker, R.S., (2000), "Effect of Partial Coating Blockage on Film Cooling Effectiveness",

Proceedings of ASME TURBOEXPO 2000, 2000-GT-0244

[20] Wang, J., Cui, P., Sunden, B., Vujanovic, M., (2016), "Effects of Deposition Height and Width on Film Cooling", Numerical Heat Transfer, Part A: Applications, 70:6, 673-687

[21] Rozati, A., Tafti, D.A., Sreedharan, S.S., (2011), "Effects of Syngas Ash Particle Size on Deposition and Erosion of a Film Cooled Leading Edge", ASME Journal of Turbomachinery, Vol. 133, 011010-1

[22] Walsh, W.S., Thole, K.A., Joe, C., (2006), "Effects of Sand Ingestion on the Blockage of FilmCooling Holes", ASME Turbo Expo 2006: Power for Land, Sea and Air, GT2006-90067

[23] Lawson, S.A., Thole, K.A., (2011), "Effects of Simulated Particle Deposition on Film Cooling", ASME Journal of Turbomachinery, Vol. 133, 021009-1

[24] Lawson, S.A., Thole, K.A., (2012), "Simulations of Multiphase Particle Deposition on Endwall Film-Cooling”, ASME Journal of Turbomachinery, Vol. 134, 011003-1

[25] Lawson, S.A., Thole, K.A., (2011), "Simulations of Multi-Phase Particle Deposition on Endwall Film-Cooling Holes in Transverse Trenches", Proceedings of ASME Turbo Expo 2011, GT2011-45190 [26] Lawson, S.A., Thole, K.A., Okita, Y., Nakamata, C., (2011), "Simulations of Multi-Phase Particle Deposition on a Showerhead with Stappered Film-Cooling Holes", Proceedings of ASME Turbo Expo 2011, GT2011-45191

[27] Ai, W., Murray, N., Fletcher, T.H., Harding, S., Lewis, S., Bons, J.P., (2012), “Deposition Near Film Cooling Holes on a High Pressure TurbineVane", ASME Journal of Turbomachinery, Vol. 134, 041013-1 [28] Ai, W., Murray, N., Fletcher, T.H., Harding, S., Bons, J.P., (2012), "Effect of Hole Spacing on Deposition of Fine Coal Flyash Near Film Cooling Holes”, ASME Journal of Turbomachinery, Vol. 134, 041021-1

[29] Cardwell, N.D., Thole, K.A., Burd, S.W., (2010), "Investigation of Sand Blocking Within Impingment and Film Cooling Holes", ASME Journal of Turbomachinery, Vol. 132, 021020-1 [30] Land, C.C., Joe, C., Thole, K.A., (2010), “Considerations of a Double-Wall Cooling Design to Reduce Sand Blockage", ASME Journal of Turbomachinery, Vol. 132, 031011-1 
[31] Jovanovic, M.B., de Lange, H.C., and van Steenhoven, A.A. (2005), "Influence of production imperfection on flow field and effectiveness in film cooling", $6^{\text {th }}$ European Conference on Turbomachinary- Fluid Dynamics and Thermodynamics: conference proceedings; 7-11 March 2005, Lille, France.

[32] Nemdili, F., Azzi, A., and Jubran, B., 2011. "Numerical Investigation of the Influence of a Hole Imperfection on Film Cooling Effectiveness". Int. J. Numer. Methods for Heat Fluid Flow., 21 (1), pp. 46-60. doi:10.1108/09615531111095067.

[33] Jovanovic, M.B., de Lange, H.C., and van Steenhoven, A.A. (2005), "Influence of Laser Drilling Imperfection on Film Cooling Performances", Proceedings of GT2005, ASME Turbo Expo 2005: Power for Land, Sea and Air.

[34] Jovanovic, M.B., de Lange, H.C., and van Steenhoven, A.A. (2008), "Effect of hole imperfection on adiabatic film cooling effectiveness", International Journal of Heat and Fluid Flow, Vol. 29, pp. 377-86. [35] Cerantola, D.J., Birk, A.M., (2017). "Numerical Investigation of a Laser-Drilled Cooling Hole", Proceedings of ASME Turbo Expo 2017: Turbomachinery Technical Conference and Exposition.

[36] H. Bharathkumar, J. Jensin Joshua, P. Booma Devi \& D. Raja Joseph (2017), “Effect of Cooling Passage Imperfection On The Flow Characteristics of Film Cooled Gas Turbine Blade", International Journal of Ambient Energy, DOI: 10.1080/01430750.2017.1399456

[37] Fric, T., Roshko, A., (1994), "Vortical Structure in the Wake of a Transverse Jet", Journal of Fluid Mechanics, Vol. 279, pp. 1-47.

[38] Haven, B.A and Kurosaka, M., (1997), "Kidney and anti-kidney vortices in crossflow jets”, J. Fluid Mech. (1997), Vol. 352, pp. 27-64.

[39] Ely, M., Jubran, B.A., (2010), “A Parametric Study on the Effect of Sister Hole Location on Active Film Cooling Flow Control", Proceedings of GT2010, ASME Turbo Expo 2010: Power for Land, Sea and Air, GT2010-22060

[40] Ely, M., Jubran, B.A., (2012), "Film Cooling from Short Holes with Sister Hole Influence”, Proceedings of GT2012, ASME Turbo Expo 2012, GT2012-68081

[41] Siavash, K., Jubran, B.A., (2014), "Film Cooling from Novel Sister Shaped Single-Hole", Proceedings of ASME Turbo Expo 2014: Turbine Technical Conference and Exposition, GT2014-25971 [42] Siavash, K., Jubran, B.A., (2015), "Numerical Evaluation of the Performance of the Sister-Shaped Single-Hole Schemes on Turbine Blade Leading Edge Film Cooling", Proceedings of ASME Turbo Expo 2015: Turbine Technical Conference and Exposition, GT2015-44121

[43] Siavash, K., Jubran, B.A., (2016), “A Numerical Evaluation of the Performance of Film Cooling from a Circular Exit Shaped Hole with Sister Holes Influence”, Heat Transfer Engineering, Vol. 37, No. 22016 
[44] Rigby, D.L., (2008), “Improved Film Cooling Effectiveness by Placing a Vortex Generator Downstream of Each Hole", Proceedings of ASME Turbo Expo 2008: Power for Land, Sea and Air, GT2008-51361

[45] Kusterer, K., Elyas, A., Bohn, D., Sugimoto, T., Tanaka, R., (2008), “Double-Jet Film-Cooling for Highly Efficient Fil-Cooling with Low Blowing Ratios", Proceedings of ASME Turbo Expo 2008: Power for Land, Sea and Air, GT2008-50073

[46] Yuen, C.H.N., Martinez-Botas, R.F., (2003), "Film Cooling Characteristics of a Single Round Hole at Various Streamwise Angles in a Crossflow: Part I Effectiveness", International Journal of Heat and Mass Transfer, 46(2), pp. 221-235.

[47] Prasad, P.V.V., Kishore, S.N., Rao, T.V., (2014), "Film Cooling Characteristics of a Single Round Hole at Various Streamwise Injection Angles", International Journal of Current Engineering and Technology, Vol.4, No.2

[48] Yavuskurt, S., Habte, M., (2008), "Effect of Computational Grid on Performance of Two-Equation Models of Turbulence for Film Cooling Applications", Proceedings of ASME Turbo Expo 2008: Power for Land, Sea and Air, GT2008-50153

[49] Harrison, K.L., Bogard, D.G., (2008), “Comparison of RANS Turbulence Models for Prediction of Film Cooling Performance", Proceedings of ASME Turbo Expo 2008: Power for Land, Sea and Air, GT2008-51423

[50] Tao, Z., Zhao, Z., Ding, S., Xu, G., Wu, H., (2009), “Suitability of Three Different Two-Equation Turbulence Models in Predicting Film Cooling Performance Over a Rotating Blade”, International Journal of Heat and Mass Transfer, Vol. 52, pp. 1268-1275.

[51] Keimasi, M.R., Taeibi-Rahni, M., (2001), "Numerical Simulation of Jets in a Crossflow Using Different Turbulence Models", AIAA Journal, Vol.39, No.12

[52] Zuniga, H.A., Kapat, J.S., (2009), "Effect of Increasing Pitch-to-Diameter Ratio on the Film Cooling Effectiveness of Shaped and Cylindrical Holes Embedded in Trenches", Proceedings of ASME Turbo Expo 2009: Power for Land, Sea and Air, GT2009-60080

[53] Zhou, W., Deng, Q., He, W., Feng, Z., (2017), "Effects of Hole Pitch to Diameter Ratio P/D of Impingement and Film Hole on Laminated Cooling Effectiveness", Proceedings of ASME Turbo Expo 2017: Turbomachinery Technical Conference and Exposition, GT2017-64566

[54] Laroch, E., (2001), "Influence of Freestream Turbulence Intensity on Cooling Effectiveness", $46^{\text {th }}$ ASME International Gas Turbine \& Aeroengine Technical Congress, Exposition and Users Symposium [55] Ely, M., Jubran, B.A., (2009), “A Numerical Study on Improving Large Angle Film Cooling Performance through the Use of Sister Holes", Numerical Heat Transfer, Part A, Vol. 55, pp. 634-653. 
[56] Russo, F., Basse, N.T., (2016), "Scaling of Turbulence Intensity for Low-Speed Flow in Smooth Pipes”, Flow Measurement and Instrumentation, Vol. 52, pp. 101-114.

[57] ANSYS, Modeling Turbulent Flows, Introductory FLUENT Training, FLUENT v6.3.

[58] Wilcox, D.C. (2006). Turbulence modeling for CFD. La Canada, CA: DCW Industries. 